\title{
Ubiquitous instabilities of dust moving in magnetized gas
}

\author{
Philip F. Hopkins` and Jonathan Squire \\ TAPIR, Mailcode 350-17, California Institute of Technology, Pasadena, CA 91125, USA
}

Accepted 2018 June 5. Received 2018 June 4; in original form 2018 January 27

\begin{abstract}
Squire \& Hopkins showed that coupled dust-gas mixtures are generically subject to 'resonant drag instabilities' (RDIs), which drive violently growing fluctuations in both. But the role of magnetic fields and charged dust has not yet been studied. We therefore explore the RDI in gas that obeys ideal MHD and is coupled to dust via both Lorentz forces and drag, with an external acceleration (e.g. gravity, radiation) driving dust drift through gas. We show this is always unstable, at all wavelengths and non-zero values of dust-to-gas ratio, drift velocity, dust charge, 'stopping time' or drag coefficient (for any drag law), or field strength; moreover, growth rates depend only weakly (sub-linearly) on these parameters. Dust charge and magnetic fields do not suppress instabilities, but give rise to a large number of new instability 'families,' each with distinct behavior. The 'MHD-wave' (magnetosonic or Alfvén) RDIs exhibit maximal growth along 'resonant' angles where the modes have a phase velocity matching the corresponding MHD wave, and growth rates increase without limit with wavenumber. The 'gyro' RDIs are driven by resonances between drift and Larmor frequencies, giving growth rates sharply peaked at specific wavelengths. Other instabilities include 'acoustic' and 'pressurefree' modes (previously studied), and a family akin to cosmic ray instabilities that appear when Lorentz forces are strong and dust streams super-Alfvénically along field lines. We discuss astrophysical applications in the warm ISM, circum-galactic medium/inter-galactic medium (CGM/IGM), H II regions, SNe ejecta/remnants, Solar corona, cool-star winds, GMCs, and AGN.
\end{abstract}

Key words: accretion, accretion discs - instabilities - turbulence-planets and satellites: formation-ISM: kinematics and dynamics - galaxies: formation.

\section{INTRODUCTION}

Almost all astrophysical fluids are laden with dust, and that dust is critical for a wide range of phenomena including planet and star formation, extinction and reddening, stellar evolution (in cool stars), astro-chemistry, feedback, and launching of winds from star-forming regions and active galactic nuclei (AGNs), the origins and evolution of heavy elements, inter-stellar gas cooling or heating, and many more. It is therefore of paramount importance to understand how dust and gas interact dynamically.

Squire \& Hopkins (2017b) (henceforth Paper I) showed that dust-gas mixtures are generically unstable to a broad class of previously unrecognized instabilities. These 'resonant drag instabilities' (RDIs) appear whenever a gas system that supports any wave (or linear perturbation/mode) with frequency $\omega_{\mathrm{g}}$ also contains dust streaming with a finite drift velocity $\mathbf{w}_{\mathrm{s}}$ (relative to the gas). Although a very broad range of wavenumbers is typically unstable, the 'resonance,' which produces the fastest growing modes, arises when $\mathbf{w}_{\mathrm{s}} \cdot \mathbf{k}=\omega_{\mathrm{g}}$ (or equivalently, the dust drift velocity in the direction of wave propagation is equal to the natural phase velocity of the wave in the gas).

Given the fact that dust is ubiquitous, and that essentially any type of gas system can meet these conditions, we expect the RDI to arise across a wide range of astrophysical contexts in the ISM, stars, galaxies, AGNs, and more. Dust is almost always expected to have some non-vanishing drift velocity owing to combinations of radiative forces on grains (e.g. absorption of light, photo-desorption, photo-electric, and Poynting-Robertson effects) or gas (e.g. line-driving the gas, pushing gas instead of dust), or gravity (which causes dust to 'settle' when the gas is pressure supported), or any hydrodynamic/pressure forces on the gas (accelerating or decelerating gas, but not [directly] dust).

Paper I briefly noted several representative examples of the RDI, where the resonance could be between gas and acoustic modes (sound waves), magnetosonic waves, Brunt-Väisälä oscillations, or epicyclic oscillations (which turns out to be the well-studied Youdin \& Goodman

^E-mail: phopkins@caltech.edu 
Table 1. Variables used throughout the text (defined here).

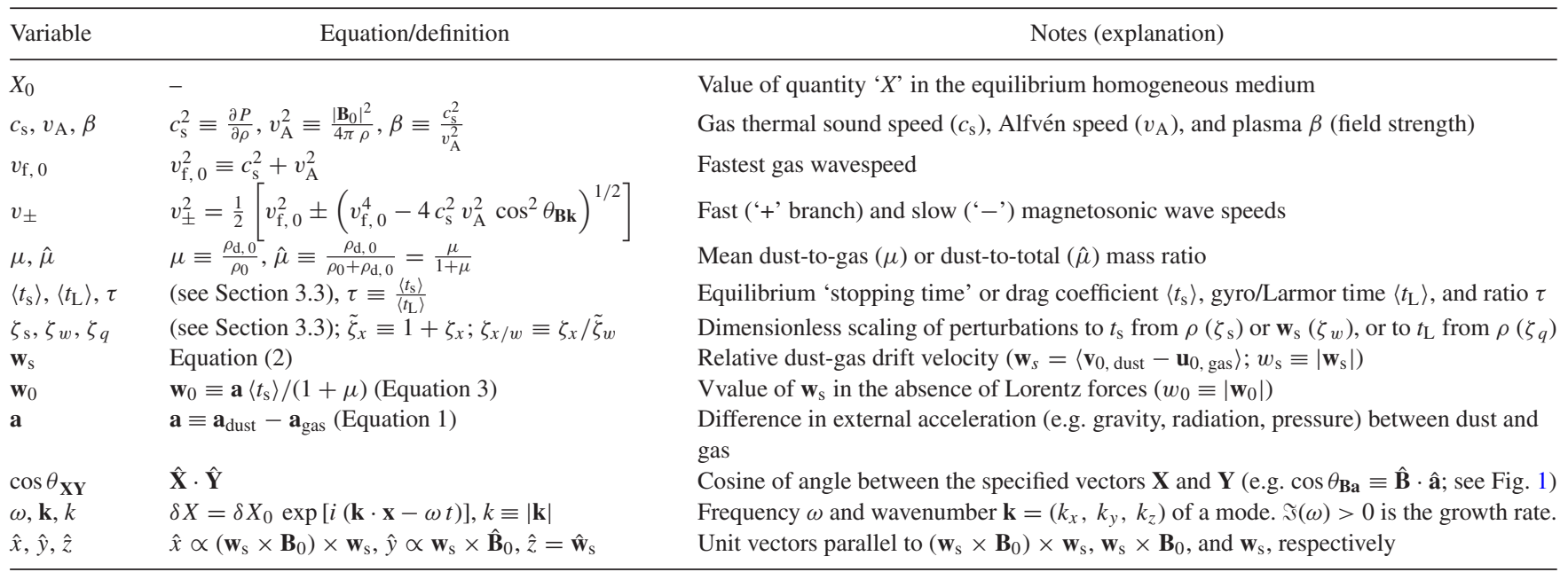

2005 'streaming instability'). Each of these modes is associated with a corresponding RDI. Many of these - particularly the epicyclic RDI and new variations with faster growth rates - are explored in more detail in the specific context of proto-planetary and proto-stellar discs, in Squire \& Hopkins (2017a). In Hopkins \& Squire (2017) (hereafter Paper II), we explored the 'acoustic RDI' in detail. This is perhaps the simplest example of the RDI - ideal, inviscid, neutral hydrodynamics, where the only wave (absent dust) is a sound wave. However, there still exists an entire family of instabilities, with a range of non-trivial scalings for their growth rates, depending on wavenumber and drift velocity. We showed that the growth rates were particularly interesting for cool-star winds, and regions of the cold, dense ISM (e.g. star-forming GMCs and 'dusty torii' around AGN). However, because there is only one wavespeed in the problem, $c_{\mathrm{s}}$, the acoustic RDI in particular requires $\left|\mathbf{w}_{\mathrm{s}}\right|$ $\geq c_{\mathrm{s}}$ for the 'resonance condition' to be met; otherwise, the system is still unstable but growth rates are significantly lower.

Of course, a huge range of astrophysical systems is ionized (at least partially), and magnetic fields cannot be neglected. This introduces two important changes to the RDI. First, even in ideal MHD with neutral dust grains, there are now three wave families: fast and slow magnetosonic waves, and Alfvén waves. Each of these has a corresponding associated family of RDIs. Second, if dust grains are charged (as they are expected to be), then at low densities and/or in sufficiently warm/hot gas, the Lorentz forces on grains can be significantly stronger than the drag forces (and, if the grains are moving sub-sonically, electrostatic Coulomb drag may dominate over collisional Epstein or Stokes drag; see e.g. Elmegreen 1979). This again introduces new families of RDIs.

Our purpose in this paper is therefore to study the linear instability of the RDI in magnetized gas, allowing for arbitrarily charged dust. We will show that all of these changes introduce new associated instabilities and behaviors of the RDI - a wide variety of previously unrecognized families of instabilities appear, each with associated resonances and different mode structure. Critically, none of these changes uniformly suppresses the RDI. In fact, we will show that the presence of slow and Alfvén waves, which have phase velocities that can become arbitrarily slow (at the appropriate propagation angles), means that the resonance condition can always be satisfied, regardless of the dust drift velocity. We will show that for any non-zero dust drift velocity $\mathbf{w}_{\mathrm{s}}$, dust-to-gas mass ratio $\mu$, magnetic field strength $\beta$, grain charge, or dust drag law, the instabilities persist and all wavelengths are unstable, with growth rates that formally become infinitely fast at small wavelengths (absent dissipative effects such as viscosity).

In Section 2, we provide a brief, high-level overview of the most important new instability families described here. Sections 3-8 are largely technical: in Section 3, we present the relevant derivation, equilibrium (background) solutions (Section 3.1), linearized equations-of-motion (Section 3.2), detailed scalings for different drag laws and Lorentz forces (Section 3.3), and the resulting dispersion relation (Section 3.4; more detail in appendices). Sections 4-6 are devoted to detailed discussion of the origins, instability conditions, resonance conditions (wavevector angles or wavelengths where growth rates are fastest), and mode structure of the different instabilities. Section 4 focuses on the families of 'parallel' pressure-free and cosmic-ray-like modes, Section 5 focuses on the MHD wave (fast and slow magnetosonic and Alfvén) RDIs, and Section 6 focuses on the gyro RDIs. Section 7 briefly notes additional modes and Section 8 discusses the range of scales where our derivations are valid. In Section 9, we discuss astrophysical applications. We first present some relevant scalings (Section 9.1) and then provide simple estimates of the growth rates and modes of greatest interest in different contexts, including the warm-ionized and warm-neutral medium (Section 9.2.1), the circum- and intergalactic medium (Section 9.2.2), H II regions (Section 9.2.3), SNe ejecta and remnants (Section 9.2.4), the Solar and stellar coronae (Section 9.2.5), cool-star winds (Section 9.2.6), the cold ISM in GMCs and around AGN (Section 9.2.7), and protoplanetary discs and planetary atmospheres (Section 9.2.8). We conclude in Section 10.

Readers primarily interested in astrophysical applications may wish to simply read the overview of the instabilities in Section 2, and then skip directly to Section 9. Table 1 defines a number of variables to which we will refer throughout the text. 


\section{OVERVIEW OF DIFFERENT INSTABILITIES}

We will show that with magnetic fields present, the dust-gas mixture gives rise to several different families of instabilities, each of which can behave differently. To guide the reader, we summarize here the modes that will be explored in detail in this paper. Variable names used here and throughout the manuscript are defined in Table 1.

\subsection{Parallel/aligned modes}

First we introduce mode families that are primarily 'parallel,' in that the fastest-growing mode has wavevector along the direction of the dust $\operatorname{drift}\left(\hat{\mathbf{k}} \approx \hat{\mathbf{w}}_{\mathrm{s}}\right)$. This is primarily of interest either (a) at very long wavelengths (low- $k$ ) at any level of magnetization or (b) at intermediate/short wavelengths when the ratio of drag stopping time to Larmor time $\left(\tau \equiv\left\langle t_{\mathrm{S}}\right\rangle /\left\langle t_{\mathrm{L}}\right\rangle\right)$ is very large, so that the dust motion (drift and perturbed velocities) becomes increasingly confined along field lines (so $\hat{\mathbf{k}} \approx \hat{\mathbf{w}}_{\mathrm{s}} \approx \hat{\mathbf{B}}_{0}$; for explanation, see equation 2 and Section 3.1).

(i) 'Pressure-free' or long-wavelength mode: At sufficiently long wavelength (low- $k$ ), the system is always unstable ${ }^{1}$ with a mode that is fastest growing in the parallel direction, with growth rate $\Im(\omega) \sim\left(\hat{\mu} w_{0}^{2} k^{2} /\left\langle t_{\mathrm{s}}\right\rangle\right)^{1 / 3}$. This is a strongly compressible, longitudinal mode $(\delta \mathbf{v}$ and $\delta \mathbf{u}$ are also aligned with $\hat{\mathbf{k}})$, which only occurs on wavelengths much larger than the dust free-streaming length and gyro radius. When the wavelength is sufficiently large, the aerodynamic force from dust on gas ('back-reaction') becomes larger than the gas pressure/magnetic forces, so this is fundamentally an instability of two frictionally coupled co-spatial pressure-free fluids, and is essentially identical in non-magnetized gas (Paper II).

(ii) 'Quasi-sound' and 'quasi-drift' modes: Even at very large $\tau$, two longitudinal, compressible modes exist when $\hat{\mathbf{k}} \approx \hat{\mathbf{B}}_{0}$, which are only weakly modified by magnetic fields. These are field-aligned acoustic modes, and have identical scalings and mode structure as shown in Paper II. The 'quasi-sound' mode is a modified sound wave, propagating at the sound speed, and is unstable when $w_{\mathrm{s}}$ is trans or super-sonic, with growth rate $\sim \mu w_{\mathrm{s}} /\left(2 c_{\mathrm{s}}\left\langle t_{\mathrm{s}}\right\rangle\right)$. The 'quasi-drift' mode is modified dust advection, propagating at $w_{\mathrm{s}}$, and is unstable for either (a) super-sonic drift or (b) sub-sonic drift if Coulomb drag dominates over Epstein drag, with growth rate $\Im(\omega) \sim\left(\mu /\left\langle t_{\mathrm{s}}\right\rangle\right) \min \left(w_{\mathrm{s}}^{2} / c_{\mathrm{s}}^{2}, 1\right)$. These are the 'out of resonance' modes that merge and become the 'fast magnetosonic' RDI at the appropriate $\hat{\mathbf{k}}$, however over some conditions they can be faster-growing than the resonant mode either because no resonant angle exists, or because non-aligned modes are suppressed by $\tau$ (and they can be faster growing than the cosmic ray-like modes below at low- $k$ or intermediate- $\tau$ ).

(iii) 'Cosmic ray streaming' mode: At large $\tau$, other parallel modes appear $\left(\hat{\mathbf{k}} \approx \hat{\mathbf{w}}_{\mathrm{s}} \approx \hat{\mathbf{B}}_{0}\right)$. These fall into two broad categories, which are manifestations of well-known resonant and non-resonant cosmic-ray instabilities (Kulsrud \& Pearce 1969; Bell 2004). The resonant variety is the high- $\tau$ limit of the gyro-resonant RDI (see Section 2.3 below). The non-resonant variety is a transverse, weakly compressible mode, featuring large transverse perturbations to the magnetic field with corresponding gyro motion of the dust and gas, as the dust drifts super-Alfvénically along the field (it is unstable for $w_{\mathrm{s}}>v_{\mathrm{A}} \hat{\mu}^{-1 / 2}$ along $\hat{\mathbf{B}}_{0}$ ). It has a growth rate $\Im(\omega) \sim k w_{\mathrm{s}} \hat{\mu}^{1 / 2}$ at large scales, a growth rate $\Im(\omega) \sim\left(\mu w_{\mathrm{s}}\left\langle t_{\mathrm{L}}\right\rangle\right)^{1 / 2} k_{z}^{1 / 2}$ for an intermediate range of scales, $\mu v_{\mathrm{A}} / w_{\mathrm{s}} \lesssim k v_{\mathrm{A}}\left\langle t_{\mathrm{L}}\right\rangle \lesssim \mu w_{\mathrm{s}} / v_{\mathrm{A}}$, and is stabilized at very short wavelengths.

\subsection{The MHD-wave RDI modes}

These are the simple RDI modes described in Section 1, which have growth rates that depend on the direction of $\hat{\mathbf{k}}$ and usually peak at the 'resonant angle' when $\omega_{\mathrm{d}}=\mathbf{w}_{\mathrm{s}} \cdot \mathbf{k}=\omega_{\mathrm{g}}=v_{\mathrm{p}}(\hat{\mathbf{k}}) k$, where $v_{\mathrm{p}}(\hat{\mathbf{k}})$ is the phase velocity of either the fast magnetosonic, slow magnetosonic, or Alfvén wave in the gas in the direction $\hat{\mathbf{k}}$ (without dust). Because $v_{p}$ depends on angle, there are a range of angles that satisfy the resonant criterion, so each wave sources a different sub-family of resonant instabilities. ${ }^{2}$ These are always unstable at all wavelengths if the resonant criterion can be satisfied. At intermediate wavelengths, ${ }^{3}$ the growth rates scale as $\sim\left(\hat{\mu} k v_{\mathrm{f}, 0} / 2\left\langle t_{\mathrm{s}}\right\rangle\right)^{1 / 2}$, and at sufficiently high $k$ as $\sim\left(\mu k v_{\mathrm{f}, 0} / 2\left\langle t_{\mathrm{s}}\right\rangle^{2}\right)^{1 / 3}$. Out of resonance the modes are still present but with (usually) lower growth rates.

(i) Fast magnetosonic RDI: Here the resonance is with the fast wave. Since this has a minimum phase $\operatorname{speed}\left|v_{\mathrm{p}}\right|=\max \left(v_{\mathrm{A}}, c_{\mathrm{s}}\right)$, the resonance condition can only be satisfied if $w_{\mathrm{s}} \geq \max \left(v_{\mathrm{A}}, c_{\mathrm{s}}\right) \sim v_{\mathrm{f}, 0}$. This is the simple MHD extension of the acoustic RDI from Paper II (it reduces to that for $\beta \rightarrow \infty$ ), and the mode structure, growth rates (above), and resonant angles ( $\left.\cos \theta_{\mathbf{w k}} \sim \pm w_{\mathrm{s}} / v_{\mathrm{f}, 0}\right)$ are very similar to the

\footnotetext{
${ }^{1}$ Technically, some modes, such as the long-wavelength mode, can be stabilized if and only if some (normally order-unity) complex pre-factor $\mathcal{C}$ (which is usually a complicated function of the various parameters here) satisfies $\|\mathcal{C}\|=0$ exactly, which is possible only for specific, singular values of certain parameters (typically the drift speed, equation of state parameter $\gamma, \tau$, and magnetic field angles must all have exactly one certain - and often un-physical value). Even then, we show in Paper II this often does not eliminate the instability, but only the leading-order term in the series expansions used to estimate the growth rates here. We will therefore simply refer to these modes as 'always unstable.'

${ }^{2}$ The forward and backward-traveling wave groups behave essentially identically for the normal MHD-Wave RDI modes, so we do not distinguish them here.

${ }^{3}$ As shown in Paper I and Paper II, technically the intermediate-wavelength ('mid- $k$ ') and short-wavelength ('high- $k$ ') RDI modes (for a given MHD wave family) are different branches of the dispersion relation, which produce faster growth rates at the same angle at mid- $k$ and high- $k$, respectively. Since their behavior is similar and the resonance condition is identical (and for some parameter choices they become degenerate at both mid- $k$ and high- $k$ ), we will refer to them for simplicity as a single mode, with different behavior in different limiting regimes.
} 
acoustic RDI after the replacement $c_{\mathrm{s}} \rightarrow v_{\mathrm{f}, 0}$. These are strongly compressible, fast-wave-like ${ }^{4}$ modes that source proportionally very large fluctuations in the dust-to-gas ratio at high- $k$ (see Paper II).

(ii) Slow magnetosonic RDI: Since the slow wave has a phase speed that vanishes as $\hat{\mathbf{k}} \cdot \hat{\mathbf{B}}_{0} \rightarrow 0$, there always exists a range of angles that satisfy the resonant condition with the slow mode (for any $\left.\mathbf{w}_{\mathrm{s}}\right)$. However, when $w_{\mathrm{s}} \ll \min \left(c_{\mathrm{s}}, v_{\mathrm{A}}\right)$, the resonant condition can only be satisfied at angles $\hat{\mathbf{k}}$ nearly perpendicular to $\hat{\mathbf{B}}_{0}$, where the phase speed is low, so the growth rates are suppressed by a factor $\sim w_{\mathrm{s}} / v_{\mathrm{f}, 0}$ (at intermediate- $k$ ) or $\sim\left(w_{\mathrm{s}} / v_{\mathrm{f}, 0}\right)^{2 / 3}$ (at high- $k$ ). The perpendicular nature also means the mid- $k$ (but not high- $k$ ) mode can be suppressed at large $\tau$ when $w_{\mathrm{s}}$ is small. This is a compressible, slow-wave-like mode that also sources proportionally large fluctuations in the dust-to-gas ratio at high- $k$.

(iii) Alfvén RDI: Like the slow mode, the phase speed of Alfvén waves also vanishes as $\hat{\mathbf{k}} \cdot \hat{\mathbf{B}}_{0} \rightarrow 0$, so there is again always a range of angles that satisfy the Alfvén RDI condition. These are Alfvén-wave-like modes, so are primarily transverse and weakly compressible in the gas (although the dust has a large $\delta \rho_{d}$ component). The transverse nature of Alfvén modes means that coupling to the dust occurs through the Lorentz force (coupling transverse field perturbations to compressible dust fluctuations), and the instability vanishes in the mid- $k$ regime if $\tau \rightarrow 0$. Interestingly, the growth rates are multiplied by $\sim w_{\mathrm{s}} / v_{\mathrm{f}, 0}$ (mid- $k$ ) or $\sim\left(w_{\mathrm{s}} / v_{\mathrm{f}, 0}\right)^{1 / 3}$ (high- $k$ ), in both super-and-sub-sonic limits, so when drift is super-sonic the growth rates can be even faster than the fast RDI (while with sub-sonic drift they are similar to the slow RDI). Also, like the slow mode, the mid- $k$ growth rates can be suppressed at large $\tau$ when $w_{\mathrm{s}} \ll \min \left(c_{\mathrm{s}}, v_{\mathrm{A}}\right)$ because the resonance requires $\hat{\mathbf{k}}$ nearly-perpendicular to $\hat{\mathbf{B}}_{0}$. However, the high- $k$ mode growth rate is enhanced by a factor $\sim \tau^{1 / 3}$ when $\tau \gg 1$ (but this may require very large $k$ to be realized).

\subsection{The gyroresonant RDI modes}

With non-zero Lorentz forces on grains, there is another dust eigenmode featuring coupled advection and gyro motion. The dispersion relation of this mode at high- $k$ or large- $\tau$ is given by $\omega_{\mathrm{d}} \sim \mathbf{w}_{\mathrm{s}} \cdot \mathbf{k} \pm\left\langle t_{\mathrm{L}}\right\rangle^{-1}$. There is thus another set of resonances, the 'gyro-resonant' RDI modes, which occur at the resonant condition $\omega_{\mathrm{d}} \sim \mathbf{w}_{\mathrm{s}} \cdot \mathbf{k} \pm\left\langle t_{\mathrm{L}}\right\rangle^{-1}= \pm v_{\mathrm{p}}(\hat{\mathbf{k}}) k$, where again $v_{\mathrm{p}}$ is the phase velocity of any of the natural gas modes (Alfvén, fast, or slow). So there are three gyro-resonant wave families, each of which can satisfy resonance for any of the four differently signed versions of the resonance equation (each of which, in turn, has a range of angles that satisfy the equation). However, when $k$ is sufficiently large, the $\tau$ term is negligible and the resonance condition reduces to $\mathbf{w}_{\mathrm{s}} \cdot \mathbf{k} \approx \pm v_{\mathrm{p}}(\hat{\mathbf{k}}) k$, i.e. these become degenerate with the MHD RDIs above. When $\left|\mathbf{w}_{\mathrm{s}} \cdot \mathbf{k} \pm\left\langle t_{\mathrm{L}}\right\rangle^{-1}\right|$ is small, terms we neglected in this dust eigenmode (discussed in detail in Section 6.1) cannot be ignored and these stabilize the mode. So the interesting behavior primarily comes from the gyroresonances when $\left\langle t_{\mathrm{L}}\right\rangle^{-1} \sim \pm v_{\mathrm{p}} k$, and instability at these resonances requires $\tau \gtrsim 1$. Thus, fundamentally unlike the MHD RDIs, for a given angle $\hat{\mathbf{k}}$ the resonance occurs at a specific wavenumber $k$.

(i) Fast gyroresonance: Because the fast-wave phase velocity $v_{\mathrm{p}} \sim v_{\mathrm{f}, 0}$, depends only weakly on angle, this resonance is sharply peaked in $k$ around $k^{-1} \approx\left\langle t_{\mathrm{L}}\right\rangle v_{\mathrm{f}, 0}$ (barring special cases where the resonance overlaps with the MHD-wave RDI angles). At the resonant wavenumber, the growth rates are $\sim \hat{\mu}^{1 / 2} /\left\langle t_{\mathrm{L}}\right\rangle$, although the growth rates are suppressed for the wave angles far from either alignment with the drift or MHD-wave RDI angles.

(ii) Slow gyroresonance: Here the phase speed varies smoothly from $v_{\mathrm{p}}=\min \left(c_{\mathrm{s}}, v_{\mathrm{A}}\right)$ for field-parallel modes to $v_{p}=0$ for fieldperpendicular modes, so at every angle $\hat{\mathbf{k}}$ the resonance occurs at a different $k^{-1} \sim\left\langle t_{\mathrm{L}}\right\rangle v_{\mathrm{p}}(\hat{\mathbf{k}})\left(\sim\left\langle t_{\mathrm{L}}\right\rangle \min \left(c_{\mathrm{s}}, v_{\mathrm{A}}\right) \hat{\mathbf{k}} \cdot \hat{\mathbf{B}}_{0}\right.$ for $\left.\operatorname{small}\left|\hat{\mathbf{k}} \cdot \hat{\mathbf{B}}_{0}\right|\right)$. For sufficiently perpendicular angles, $\left|\hat{\mathbf{k}} \cdot \hat{\mathbf{B}}_{0}\right| \ll w_{\mathrm{s}} / \min \left(c_{\mathrm{s}}, v_{\mathrm{A}}\right)$, this implies sufficiently large $k$ such that the mode becomes degenerate with the high- $k$ RDI. The growth rates around resonance scale similarly to the fast gyro-resonance at sufficiently high- $k$, but the suppression factor for modes perpendicular to the dust-drift means that gyro-modes that simultaneously satisfy the slow-wave RDI condition are less interesting. At sufficiently sub-Alfvénic drift velocities $w_{\mathrm{s}} \ll v_{\mathrm{A}}$, the slow gyro-RDI becomes stable for most angles.

(iii) Alfvén gyroresonance: The Alfvén-wave phase velocity is $v_{\mathrm{p}}=v_{\mathrm{A}} \hat{\mathbf{k}} \cdot \hat{\mathbf{B}}_{0}$, so again, the resonant wavenumber, $k^{-1} \sim\left\langle t_{\mathrm{L}}\right\rangle v_{\mathrm{A}} \hat{\mathbf{k}} \cdot \hat{\mathbf{B}}_{0}$, depends relatively strongly on mode angle, $\hat{\mathbf{k}}$. For angles $\left|\hat{\mathbf{k}} \cdot \hat{\mathbf{B}}_{0}\right| \ll w_{\mathrm{s}} / v_{\mathrm{A}}$ this goes to high- $k$ and becomes degenerate with the high- $k$ RDI. The scaling of the growth rate is similar to the slow-gyro RDI above.

\section{BASIC EQUATIONS AND LINEAR PERTURBATIONS}

\subsection{Equations solved and equilibrium solution}

As in Paper II, consider a mixture of gas and a second component that can be approximated as a pressure-free fluid, which we will refer to as 'dust' henceforth (see e.g. Youdin \& Goodman 2005 and appendix A of Jacquet, Balbus \& Latter 2011, as well as Section 8 below). We consider

\footnotetext{
${ }^{4}$ By 'fast-wave-like,' we mean that the mode structure/eigenfunction resembles a fast wave in both the dust and the gas to leading order, with approximately the usual ratios and phases of the density $(\delta \rho)$, longitudinal (acoustic/pressure) velocity $(\delta \mathbf{u} \cdot \hat{\mathbf{k}})$, and transverse (electromagnetic/tension) velocity $\left(\delta \mathbf{u} \cdot \hat{\mathbf{B}}_{0}\right.$ and $\delta \mathbf{B}$ in the direction perpendicular $\hat{\mathbf{k}}$ ) perturbations. The presence of drift and Lorentz forces adds a perturbation to these, and to terms that normally vanish (e.g. $\delta \mathbf{u}$ and $\delta \mathbf{B}$ in the mutually perpendicular direction $\mathbf{k} \times \mathbf{B}$ ), and (more importantly) introduces a phase offset between the dust and gas perturbations, which drives the growth of the instability (see Section 5.5).
} 
a magnetized gas that obeys the ideal MHD equations, ${ }^{5}$ and dust that feels both a generalized arbitrary drag force (e.g. neutral/aerodynamic and/or Coulomb drag) and Lorentz forces from the magnetic fields. The system is described by the conservation equations:

$$
\begin{aligned}
\frac{\partial \rho}{\partial t}+\nabla \cdot(\mathbf{u} \rho) & =0 \\
\left(\frac{\partial}{\partial t}+\mathbf{u} \cdot \nabla\right) \mathbf{u} & =-\frac{\nabla P}{\rho}-\frac{1}{4 \pi \rho} \mathbf{B} \times(\nabla \times \mathbf{B})+\mathbf{g}+\frac{\rho_{\mathrm{d}}}{\rho}\left[\frac{(\mathbf{v}-\mathbf{u})}{t_{\mathrm{s}}}+\frac{(\mathbf{v}-\mathbf{u}) \times \hat{\mathbf{B}}}{t_{\mathrm{L}}}\right] \\
\frac{\partial \mathbf{B}}{\partial t} & =\nabla \times(\mathbf{u} \times \mathbf{B}) \\
\nabla \cdot \mathbf{B} & =0 \\
\frac{\partial \rho_{\mathrm{d}}}{\partial t}+\nabla \cdot\left(\mathbf{v} \rho_{\mathrm{d}}\right) & =0 \\
\left(\frac{\partial}{\partial t}+\mathbf{v} \cdot \nabla\right) \mathbf{v} & =\mathbf{g}+\mathbf{a}-\left[\frac{(\mathbf{v}-\mathbf{u})}{t_{\mathrm{s}}}+\frac{(\mathbf{v}-\mathbf{u}) \times \hat{\mathbf{B}}}{t_{\mathrm{L}}}\right]
\end{aligned}
$$

Here $(\rho, \mathbf{u})$ and $\left(\rho_{\mathrm{d}}, \mathbf{v}\right)$ are the (density, velocity) of the gas and dust, respectively, and $P, \mathbf{B}$ are the gas (thermal) pressure and magnetic field, respectively. Throughout this manuscript $\hat{\mathbf{x}} \equiv \mathbf{x} /|\mathbf{x}|$ denotes the unit vector in direction $\mathbf{x}$. The external acceleration of the gas is $\mathbf{g}$, while $\mathbf{g}$ $+\mathbf{a}$ is the external acceleration of dust (i.e. $\mathbf{a}$ is any difference in the dust versus gas acceleration). The dust experiences a drag acceleration $\mathbf{a}_{\text {drag }}=-(\mathbf{v}-\mathbf{u}) / t_{\mathrm{s}}$ where $t_{\mathrm{s}}$ is the arbitrary drag coefficient or 'stopping time' (which is generally a function of other properties; see below). Similarly the dust, if it charged, feels a Lorentz force with acceleration $\mathbf{a}_{\text {Lorentz }}=-(\mathbf{v}-\mathbf{u}) \times \hat{\mathbf{B}} / t_{\mathrm{L}}$, where we define the Larmor/gyro time $t_{\mathrm{L}}=m_{\text {grain }} c /\left|q_{\text {grain }} \mathbf{B}\right|$ in terms of the individual dust grain's charge $\left(q_{\text {grain }}\right)$ and mass $\left(m_{\text {grain }}\right)$, and the speed-of-light $c$ (the sign convention here is arbitrary but convenient, so $\left.t_{\mathrm{L}}>0\right)^{6}{ }^{6}$

Equation (1) has the spatially homogeneous, steady-state solution:

$$
\begin{aligned}
\rho^{h} & =\langle\rho\rangle=\rho_{0}, \\
\rho_{\mathrm{d}}^{h} & =\left\langle\rho_{\mathrm{d}}\right\rangle=\rho_{d, 0} \equiv \mu \rho_{0}, \\
t_{\mathrm{s}}^{h} & =\left\langle t_{\mathrm{s}}\right\rangle=t_{\mathrm{s}}^{h}\left(\rho^{h}, \mathbf{w}_{\mathrm{s}}, \ldots\right), \\
t_{\mathrm{L}}^{h} & =\left\langle t_{\mathrm{L}}\right\rangle=t_{\mathrm{L}}^{h}\left(\rho^{h}, \mathbf{w}_{\mathrm{s}}, \ldots\right) \equiv \tau^{-1}\left\langle t_{\mathrm{s}}\right\rangle, \\
\mathbf{B}^{h} & =\langle\mathbf{B}\rangle=\mathbf{B}_{0}, \\
\mathbf{u}^{h} & =\langle\mathbf{u}\rangle=\mathbf{u}_{0}+\left[\mathbf{g}+\mathbf{a}\left(\frac{\mu}{1+\mu}\right)\right] t, \\
\mathbf{v}^{h} & =\langle\mathbf{v}\rangle=\langle\mathbf{u}\rangle+\mathbf{w}_{\mathrm{s}}, \\
\mathbf{w}_{\mathrm{s}} & \equiv \frac{|\mathbf{a}|\left\langle t_{s}\right\rangle}{1+\mu}\left[\frac{\hat{\mathbf{a}}-\tau\left(\hat{\mathbf{a}} \times \hat{\mathbf{B}}_{0}\right)+\tau^{2}\left(\hat{\mathbf{a}} \cdot \hat{\mathbf{B}}_{0}\right) \hat{\mathbf{B}}_{0}}{1+\tau^{2}}\right] .
\end{aligned}
$$

In equilibrium, the system features the dust and gas both moving with constant acceleration $\tilde{\mathbf{a}} \equiv \mathbf{g}+\mathbf{a} \mu /(1+\mu)$, and a constant relative drift velocity $\mathbf{w}_{\mathrm{s}}$. The total mass-ratio between dust and gas is defined as $\mu \equiv\left\langle\rho_{d}\right\rangle /\langle\rho\rangle$, and $\left\langle t_{\mathrm{s}}\right\rangle,\left\langle t_{\mathrm{L}}\right\rangle$ are the values of $t_{\mathrm{s}}$ and $t_{\mathrm{L}}$ in the homogeneous solution (note these can, in principle, depend on $w_{\mathrm{s}} \equiv\left|\mathbf{w}_{\mathrm{s}}\right|$, as discussed below, which makes equation (2) a non-linear equation for $\mathbf{w}_{\mathrm{s}}$ ). The parameter $\tau \equiv\left\langle t_{\mathrm{S}}\right\rangle /\left\langle t_{\mathrm{L}}\right\rangle$ is introduced here for convenience.

Some examples of these equilibrium solutions, illustrating the geometry of the problem and dependence on $\tau$, are shown in Fig. 1. The scaling of $\mathbf{w}_{\mathrm{s}}$ with $\tau$ is intuitive: for small $\tau$, i.e. $t_{\mathrm{s}} \ll t_{\mathrm{L}}$, drag dominates, and $\mathbf{w}_{\mathrm{s}} \approx \mathbf{a}\left\langle t_{\mathrm{s}}\right\rangle /(1+\mu)$, which is just the solution from Paper II for the 'terminal velocity' in a system with aerodynamic drag only (neglecting Lorentz forces). For large $\tau$, i.e. $t_{\mathrm{S}} \gg t_{\mathrm{L}}$, Lorentz forces suppress motion perpendicular to the field, giving $\mathbf{w}_{\mathrm{s}} \approx\left(\mathbf{a} \cdot \hat{\mathbf{B}}_{0}\right) \hat{\mathbf{B}}_{0}\left\langle t_{\mathrm{s}}\right\rangle /(1+\mu)$, i.e. the drift is given only by the projection of the differential acceleration a onto the field direction $\hat{\mathbf{B}}_{0}$. It is useful below to define a parameter $\mathbf{w}_{0}$ as the drift velocity in the absence of Lorentz forces $(\tau$ $\rightarrow 0$ ), giving:

$\mathbf{w}_{0} \equiv \frac{\mathbf{a}\left\langle t_{\mathrm{s}}\right\rangle}{1+\mu} \quad \mathbf{w}_{\mathrm{s}}=\frac{\mathbf{w}_{0}-\tau\left(\mathbf{w}_{0} \times \hat{\mathbf{B}}_{0}\right)+\tau^{2}\left(\mathbf{w}_{0} \cdot \hat{\mathbf{B}}_{0}\right) \hat{\mathbf{B}}_{0}}{1+\tau^{2}}$.

Note $\hat{\mathbf{w}}_{0}=\hat{\mathbf{a}}$, so when $\tau \ll 1$ we have $\mathbf{w}_{\mathrm{s}} \approx \mathbf{w}_{0}$ and $\hat{\mathbf{w}}_{\mathrm{s}} \approx \hat{\mathbf{a}}$ (so e.g. $\cos \theta_{\mathbf{B w}} \equiv \hat{\mathbf{B}} \cdot \hat{\mathbf{w}}_{s} \approx \cos \theta_{\mathbf{B a}}=\hat{\mathbf{B}} \cdot \hat{\mathbf{a}}$, etc.). Accounting for finite $\tau$, we have $\cos \theta_{\mathbf{B w}}=\cos \theta_{\mathbf{B a}}\left[\left(1+\tau^{2}\right) /\left(1+\tau^{2} \cos ^{2} \theta_{\mathbf{B a}}\right)\right]^{1 / 2}$ and $\sin \theta_{\mathbf{B w}}=\sin \theta_{\mathbf{B a}}\left[1+\tau^{2} \cos ^{2} \theta_{\mathbf{B a}}\right]^{-1 / 2}$, which for $\tau \gg 1$ become $\cos \theta_{\mathbf{B w}} \rightarrow 1$, $\sin \theta_{\mathbf{B w}} \rightarrow(1 / \tau) \tan \theta_{\mathbf{B a}}$. These relations will prove useful below.

\footnotetext{
${ }^{5}$ Non-ideal and kinetic effects, as well as the effects of current carried by grains in the induction equation, are briefly discussed in Section 8 , where we show they are negligible for many astrophysical cases discussed in Section 9.

${ }^{6}$ In equation (1), taking $q_{\text {grain }} \rightarrow-q_{\text {grain }}$ is mathematically identical to taking $\hat{\mathbf{B}} \rightarrow-\hat{\mathbf{B}}$. Since we will consider all possible signs/directions of $\mathbf{B}$, we can define $t_{\mathrm{L}}$ in terms of $\left|q_{\text {grain }} \mathbf{B}\right|$ to be positive definite, without loss of generality.
} 


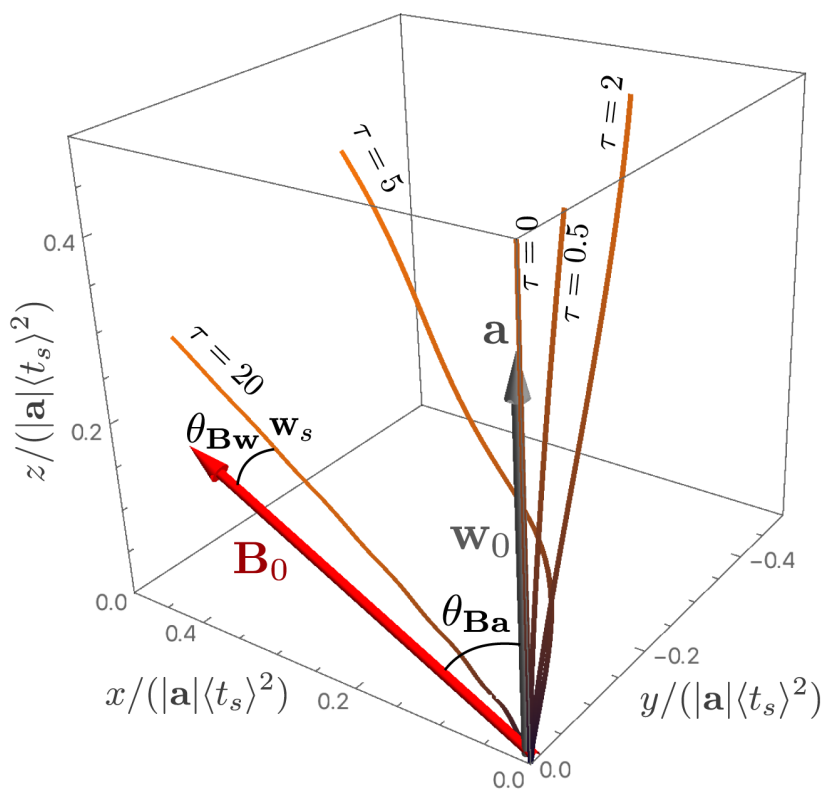

Figure 1. Illustration of the geometry of charged dust grains accelerated by a force (e.g. gravity, radiation pressure, etc., producing a) at some angle to the magnetic field $\left(\mathbf{B}_{0}\right)$. Lines show the trajectories of dust at different $\tau=\left\langle t_{\mathrm{s}}\right\rangle /\left\langle t_{\mathrm{L}}\right\rangle$ (ratio of dust drag stopping time $t_{\mathrm{S}}$ to gyro/Larmor time $t_{\mathrm{L}}$ ), accelerated from rest at the origin (we assume constant $t_{\mathrm{s}}$ for simplicity, rather than, for example, Epstein drag). The line color indicates the time along the trajectory from $t=0$ (dark brown) to $t=2.5\left\langle t_{\mathrm{s}}\right\rangle$ (orange). The equilibrium drift velocity is $\mathbf{w}_{\mathrm{s}}$, while $\mathbf{w}_{0}$ is the drift velocity in the absence of magnetic (Lorentz) forces on grains $(\tau=0)$. We also indicate, with the gray and orange arrows, respectively, the acceleration direction $\mathbf{a}\left(\right.$ with $\left.\hat{\mathbf{w}}_{0}=\hat{\mathbf{a}}\right)$ and the magnetic field direction. Some relevant angles defined in the text, $\theta_{\mathbf{B} \text { a }}$ and $\theta_{\mathbf{B} \mathbf{w}}$ (for the $\tau=20$ trajectory), are also marked. We see that the dust quickly (by $t \sim\left\langle t_{\mathrm{s}}\right\rangle$, or even faster for $\tau \gg$ 1) reaches the equilibrium trajectory given in equation (3), with drift velocity $\mathbf{w}_{\mathbf{s}}$, which is preferentially aligned with the magnetic field at large- $\tau$.

\subsection{Linearized perturbation equations}

Now consider small perturbations $\delta: \rho=\rho^{h}+\delta \rho$, etc., and boost to a free-falling frame moving with the homogeneous gas solution $\langle\mathbf{u}\rangle$. Linearizing equation (1), we obtain

$$
\begin{aligned}
\frac{\partial \delta \rho}{\partial t}= & -\rho_{0} \nabla \cdot \delta \mathbf{u}, \\
\frac{\partial \delta \mathbf{u}}{\partial t}= & -c_{\mathrm{s}}^{2} \frac{\nabla \delta \rho}{\rho_{0}}-\frac{\mathbf{B}_{0} \times(\nabla \times \delta \mathbf{B})}{4 \pi \rho_{0}}+\mu\left[\frac{(\delta \mathbf{v}-\delta \mathbf{u})}{\left\langle t_{\mathrm{s}}\right\rangle}+\frac{(\delta \mathbf{v}-\delta \mathbf{u}) \times \hat{\mathbf{B}}_{0}}{\left\langle t_{\mathrm{L}}\right\rangle}+\frac{\mathbf{w}_{\mathrm{s}} \times \delta \mathbf{B}}{\left|\mathbf{B}_{0}\right|\left\langle t_{\mathrm{L}}\right\rangle}\right]-\mu \frac{\mathbf{w}_{\mathrm{s}}}{\left\langle t_{\mathrm{s}}\right\rangle}\left(\frac{\delta t_{\mathrm{s}}}{\left\langle t_{\mathrm{s}}\right\rangle}+\frac{\delta \rho}{\rho_{0}}-\frac{\delta \rho_{d}}{\mu \rho_{0}}\right) \\
& -\mu \frac{\mathbf{w}_{\mathrm{s}} \times \hat{\mathbf{B}}_{0}}{\left\langle t_{\mathrm{L}}\right\rangle}\left(\frac{\delta t_{L}\left[\mathbf{B}_{0}\right]}{\left\langle t_{\mathrm{L}}\right\rangle}+\frac{\delta \rho}{\rho_{0}}-\frac{\delta \rho_{d}}{\mu \rho_{0}}\right), \\
\frac{\partial \delta \mathbf{B}}{\partial t}= & \nabla \times\left(\delta \mathbf{u} \times \mathbf{B}_{0}\right)=\left(\mathbf{B}_{0} \cdot \nabla\right) \delta \mathbf{u}-\mathbf{B}_{0}(\nabla \cdot \delta \mathbf{u}), \\
\frac{\partial \delta \rho_{d}}{\partial t}= & -\left(\mathbf{w}_{\mathrm{s}} \cdot \nabla\right) \delta \rho_{d}-\mu \rho_{0} \nabla \cdot \delta \mathbf{v}, \\
\frac{\partial \delta \mathbf{v}}{\partial t}= & -\left(\mathbf{w}_{\mathrm{s}} \cdot \nabla\right) \delta \mathbf{v}-\frac{(\delta \mathbf{v}-\delta \mathbf{u})}{\left\langle t_{\mathrm{s}}\right\rangle}+\frac{\mathbf{w}_{s} \delta t_{\mathrm{s}}}{\left\langle t_{\mathrm{s}}\right\rangle^{2}}-\frac{(\delta \mathbf{v}-\delta \mathbf{u}) \times \hat{\mathbf{B}}_{0}}{\left\langle t_{\mathrm{L}}\right\rangle}-\frac{\mathbf{w}_{\mathrm{s}} \times \delta \mathbf{B}}{\left|\mathbf{B}_{0}\right|\left\langle t_{\mathrm{L}}\right\rangle}+\frac{\left(\mathbf{w}_{\mathrm{s}} \times \hat{\mathbf{B}}_{0}\right) \delta t_{\mathrm{L}}\left[\mathbf{B}_{0}\right]}{\left\langle t_{L}\right\rangle^{2}},
\end{aligned}
$$

(plus the constraint $\nabla \cdot \delta \mathbf{B}=0$ ), where we define the usual sound speed $c_{\mathrm{s}}^{2} \equiv \partial P / \partial \rho$ and $\delta t_{\mathrm{s}}$ as the linearized perturbation to $t_{\mathrm{s}}$; i.e. $t_{\mathrm{S}} \equiv\left\langle t_{\mathrm{s}}\right\rangle+\delta t_{\mathrm{s}}(\delta \rho, \delta \mathbf{v}, \ldots)+\mathcal{O}\left(\delta^{2}\right)$. All variables now refer to their values in the free-falling frame. The term $\delta t_{\mathrm{L}}\left[\mathbf{B}_{0}\right]$ is defined for convenience here as the linearized perturbation to $t_{\mathrm{L}}$ at fixed magnetic field $\mathbf{B}=\mathbf{B}_{0}$, so it applies just to the scalar normalization of the Lorentz force (essentially, $\delta t_{\mathrm{L}}\left[\mathbf{B}_{0}\right]$ captures any linear variation in the grain charge). The explicit dependence of the Lorentz force on $\mathbf{B}$ is written separately, as the $\mathbf{w}_{\mathrm{s}} \times \delta \mathbf{B}$ terms.

Note that, as shown in detail in Paper I (Section 2 and appendix B therein), the transformation between accelerating frames (moving with the homogeneous gas solution) and stationary frames has no effect on the solutions here. In particular, transforming our Fourier solutions back to the stationary frame is mathematically equivalent to taking $\omega \rightarrow \omega+\mathbf{u}_{0} \cdot \mathbf{k}+\tilde{\mathbf{a}} \cdot \mathbf{k} t / 2$ (where $\tilde{\mathbf{a}}=\mathbf{g}+\mathbf{a} \mu /(1+\mu)$ is the homogeneous acceleration). 


\subsection{Scalings of the stopping time and gyro time}

To define $\delta t_{\mathrm{s}}$ we require a physical drag law. However, as shown in Paper II, essentially all physical drag laws $t_{\mathrm{s}}=t_{\mathrm{s}}\left(\rho, T, c_{\mathrm{s}}, P, \mathbf{v}-\mathbf{u}, \ldots\right)$ can be written, assuming a barytropic equation of state under local perturbations, in the form:

$\frac{\delta t_{\mathrm{s}}}{\left\langle t_{\mathrm{s}}\right\rangle}=-\zeta_{\mathrm{s}} \frac{\delta \rho}{\rho_{0}}-\zeta_{w} \frac{\mathbf{w}_{\mathrm{s}} \cdot(\delta \mathbf{v}-\delta \mathbf{u})}{\left|\mathbf{w}_{\mathrm{s}}\right|^{2}}$.

Likewise we expect variations of the gyro timescale (at fixed $\mathbf{B}$ ) to have the form:

$\frac{\delta t_{\mathrm{L}}\left[\mathbf{B}_{0}\right]}{\left\langle t_{\mathrm{L}}\right\rangle}=-\zeta_{q} \frac{\delta \rho}{\rho_{0}}$

To justify this and explore scalings in different physical regimes, we briefly describe different physical scaling laws below.

\subsubsection{Epstein drag}

For Epstein drag, valid for grain sizes smaller than the gas mean-free-path (for both super-sonic and sub-sonic drift velocities), the stopping time is $t_{\mathrm{s}}=(\pi \gamma / 8)^{1 / 2}\left(\bar{\rho}_{\mathrm{d}} R_{\mathrm{d}} / \rho c_{\mathrm{s}}\right)\left(1+a_{\gamma}|\mathbf{v}-\mathbf{u}|^{2} / c_{\mathrm{s}}^{2}\right)^{-1 / 2}$ (Draine \& Salpeter 1979b). Here $\bar{\rho}_{d}$ is the internal material density of the particle (grain), $R_{\mathrm{d}}$ is the grain radius, $a_{\gamma} \equiv 9 \pi \gamma / 128$, and $\gamma$ is the equation-of-state parameter, needed to relate $c_{\mathrm{s}}$ to the isothermal sound speed $c_{\text {iso }}$ (or temperature $T$ ):

$\gamma \equiv \frac{c_{\mathrm{s}}^{2}}{c_{\mathrm{iso}}^{2}}=\frac{\rho}{P} \frac{\partial P}{\partial \rho}$.

Evaluating equation (5), one finds

$\zeta_{\mathrm{s}}^{\text {Epstein }}=\frac{\gamma+1+2 \tilde{a}_{E}}{2\left(1+\tilde{a}_{E}\right)} \quad \zeta_{w}^{\text {Epstein }}=\frac{\tilde{a}_{E}}{1+\tilde{a}_{E}}$,

where $\tilde{a}_{E} \equiv a_{\gamma}\left(w_{\mathrm{s}} / c_{\mathrm{s}}\right)^{2}$

As discussed in Paper II, because $\left\langle t_{\mathrm{s}}\right\rangle$ (and therefore also $\tau$ ) depends explicitly on $\langle\mathbf{v}-\mathbf{u}\rangle=\mathbf{w}_{\mathrm{s}}$, equation (2) for the drift velocity (or equation 3 for $\mathbf{w}_{0}$ ) must be solved implicitly to determine the equilibrium $\mathbf{w}_{\mathrm{s}}$, if one is given some $\mathbf{a}$ (and $\rho, R_{\mathrm{d}}$, etc). If we define $t_{0} \equiv(\pi \gamma / 8)^{1 / 2} \bar{\rho}_{\mathrm{d}} R_{\mathrm{d}} /\left(\rho_{0} c_{s}\right)$ as the stopping time neglecting the $\mathbf{v}-\mathbf{u}$ term, then if the drift is sub-sonic - that is, if $\left|\mathbf{w}_{0}\left(t_{\mathrm{s}}=t_{0}\right)\right|=$ $|\mathbf{a}| t_{0} /(1+\mu) \ll c_{\mathrm{s}}-$ then $\mathbf{w}_{\mathrm{s}} \approx \mathbf{w}_{\mathrm{s}}\left(t_{\mathrm{s}}=t_{0}\right)$; if the drift is highly super-sonic and either $\tau \ll 1$ or $\tau \gg 1$, then one obtains $w_{\mathrm{s}} \propto\left[c_{\mathrm{s}} w_{s}\left(t_{\mathrm{s}}=t_{0}\right)\right]^{1 / 2}$.

\subsubsection{Stokes drag}

The expression for drag in the Stokes limit - which is valid for an intermediate range of grain sizes, when $R_{\mathrm{d}} \gtrsim(9 / 4) \lambda_{\text {mfp }}$ but Re grain $\equiv$ $R_{\mathrm{d}}\left|\mathbf{w}_{\mathrm{s}}\right| /\left(\lambda_{\mathrm{mfp}} c_{\mathrm{s}}\right) \lesssim 1-$ is given by multiplying the Epstein expression above by $\left(4 R_{\mathrm{d}}\right) /\left(9 \lambda_{\mathrm{mfp}}\right)$. Here $\lambda_{\text {mfp }} \propto 1 /\left(\rho \sigma_{\text {gas }}\right)$ is the gas mean-free-path, $\sigma_{\text {gas }}$ is the gas collision cross-section, and $\mathrm{Re}_{\text {grain }}$ is the Reynolds number of the streaming grain. This gives

$\zeta_{\mathrm{s}}^{\text {Stokes }}=\zeta_{\mathrm{s}}^{\text {Epstein }}-1 \quad \zeta_{w}^{\text {Stokes }}=\zeta_{w}^{\text {Epstein }}$.

As discussed in Paper II, this assumes $\sigma_{\text {gas }}$ is not strongly dependent on density or temperature. Of course under some circumstances, given a specific physical model/system, it should be, in which case one can easily calculate the appropriate revised $\zeta_{s}$. Note the dependence of $t_{s}$ on $\mathbf{v}-\mathbf{u}$ is the same as Epstein, so equation (2) must be solved non-linearly for a given $\mathbf{a}$.

\subsubsection{Coulomb drag}

The stopping time for Coulomb drag can be approximated as (Draine \& Salpeter 1979b),

$t_{\mathrm{s}}=\left(\frac{\pi \gamma}{2}\right)^{1 / 2} \frac{\bar{\rho}_{\mathrm{d}} R_{\mathrm{d}}}{f_{\text {ion }} \rho c_{\mathrm{s}} \ln \Lambda}\left(\frac{k_{B} T}{z_{i} e U}\right)^{2}\left(1+a_{C} \frac{|\mathbf{v}-\mathbf{u}|^{3}}{c_{\mathrm{s}}^{3}}\right)$,

where $\ln \Lambda$ is a Coulomb logarithm with $\Lambda \equiv\left[\left(3 k_{B} T\right) /\left(2 R_{d} z_{i} e^{2} U\right)\right]\left[\left(\mu_{i} m_{p} k_{B} T\right) /\left(\pi f_{\text {ion }} \rho\right)\right]^{1 / 2}, a_{\mathrm{C}} \equiv \sqrt{2 \gamma^{3} / 9 \pi}, e$ is the electron charge, $f_{\text {ion }}$ the ionized fraction (in the gas), $z_{\mathrm{i}}$ is the mean gas ion charge, $\mu_{\mathrm{i}}$ is the mean molecular weight of ions, $T \propto \rho^{\gamma-1}$ is the gas temperature, and $U$ is the electrostatic potential of the grains, $U \sim Z_{\text {grain }} e / R_{\mathrm{d}}$ (where $Z_{\text {grain }}$ is the grain charge). The behavior of $U$ is complicated and depends on a wide variety of environmental factors: in the different regimes considered in Draine \& Salpeter (1979b), they find regimes where $U \sim$ constant and others where $U \propto Z_{\text {grain }} \propto T$, we therefore parametrize the dependence by $U \propto T^{\zeta C}$. This gives

$$
\begin{aligned}
\zeta_{s}^{\text {Coulomb }} & =1+2(\gamma-1) \zeta_{\mathrm{C}}-\frac{3(\gamma-1)}{2\left(1+\tilde{a}_{\mathrm{C}}\right)}-\frac{1-\left(3-2 \zeta_{\mathrm{C}}\right)(\gamma-1)}{2 \ln \Lambda}, \\
\zeta_{w}^{\text {Coulomb }} & =-\frac{3 \tilde{a}_{\mathrm{C}}}{1+\tilde{a}_{\mathrm{C}}}
\end{aligned}
$$


where $\tilde{a}_{\mathrm{C}} \equiv a_{\mathrm{C}}\left(w_{\mathrm{s}} / c_{\mathrm{s}}\right)^{3}$. For relevant astrophysical conditions, $\ln \Lambda \sim 15-20$, so the $\ln \Lambda$ term in $\zeta_{\mathrm{s}}$ is unimportant. As discussed in Paper II, when $w_{\mathrm{s}} \gg c_{\mathrm{s}}$, Coulomb drag produces a new instability (in the absence of other drag forces), because of the large inverse dependence of $t_{\mathrm{s}}$ on $\mathbf{v}-\mathbf{u}\left(\zeta_{w}<-1\right.$, here); however for precisely this reason aerodynamic drag (Epstein or Stokes drag) will always dominate over Coulomb drag in this limit (the aerodynamic term becomes stronger, while the Coulomb term becomes weaker, as $w_{\mathrm{s}}$ increases).

\subsubsection{Lorentz forces and gyro timescales}

The Larmor/gyro time, $t_{\mathrm{L}}\left[\mathbf{B}_{0}\right]$, is

$t_{\mathrm{L}} \equiv-\frac{m_{\text {grain }} c}{\left|q_{\text {grain }} \mathbf{B}\right|}=\frac{4 \pi \bar{\rho}_{d} R_{d}^{3} c}{3 e\left|Z_{\text {grain }} \mathbf{B}\right|}$.

As noted above, the grain charge distribution is complicated and determined by a range of physical processes, environmental effects, and microphysical grain properties (see Weingartner \& Draine 2001a,c). Our analysis allows for an arbitrary dependence of $Z_{\text {grain }}$ on these properties, but to gain some intuition we note that the dominant processes are usually 'collisional' and/or photo-electric charging (Tielens 2005). In the collision-dominated regime (grains 'sweeping up' electrons), Draine \& Sutin (1987) quote convenient results for the equilibrium charge as a function of temperature: $\left\langle Z_{\text {grain }}\right\rangle \approx-1 /\left(1+0.037 \tilde{T}^{-1 / 2}\right)-a_{\mathrm{L}} \tilde{T} \approx-1-a_{\mathrm{L}} \tilde{T}$ where $\tilde{T} \equiv R_{\mathrm{d}} k_{\mathrm{B}} T / e^{2}$ and $1+a_{\mathrm{L}}=\left(\mu / m_{e}\right)^{1 / 2} \exp \left(-a_{\mathrm{L}}\right)$, with a minimum charge limited by field emission $Z_{\min } \sim-7000\left(R_{d} / 0.1 \mu \mathrm{m}\right)^{2}$. Here $\mu$ is the mean molecular weight; for typical values in ionized (WIM), atomic (WNM/CNM), or molecular media, relevant values are $\left(\mu, a_{\mathrm{L}}\right) \approx(0.59,2.3),(1.25,2.6)$, and $(2.3,2.8)$, respectively. In the photo-electric (electron-ejection dominated) regime, $\left\langle Z_{\text {grain }}\right\rangle \approx-1+\tilde{T}\left(f_{\mathrm{L}}-1\right)$ where $f_{\mathrm{L}} \equiv\left(F_{\text {pe }} / 2.6 \times\right.$ $\left.10^{-4} \mathrm{erg} \mathrm{cm}^{-2} \mathrm{~s}^{-1}\right)\left(n_{e} / \mathrm{cm}^{-3}\right)^{-1}(T / \mathrm{K})^{-1 / 2}$ and $F_{\mathrm{pe}}$ is the incident UV flux in the relevant (far-UV) wavelength range (Tielens 2005), with maximum charge above which electrostatic forces prevent ejection, $Z_{\max } \sim 500\left(R_{d} / 0.1 \mu \mathrm{m}\right)$.

For large grains $\left(\left|Z_{\text {grain }}\right| \gg 1\right)$, between minimum/maximum charge, one can approximately interpolate between the regimes by taking $\left\langle Z_{\text {grain }}\right\rangle \sim-1+\tilde{T}\left(f_{\mathrm{L}}-a_{\mathrm{L}}\right)$. If the grain-charging timescale $t_{\text {charge }}$ is also short compared to the stopping time and other dynamical timescales in the system (not always guaranteed; see Section 8), then this gives

$\zeta_{q} \approx \frac{\tilde{T}_{0}\left[a_{\mathrm{L}}(\gamma-1)+f_{\mathrm{L}}(3-\gamma) / 2\right]}{1+\left(a_{\mathrm{L}}-f_{\mathrm{L}}\right) \tilde{T}_{0}} \sim\left\{\begin{array}{lc}(\gamma-1) & \left(f_{\mathrm{L}} \ll 1 ; \text { collision }- \text { dominated }\right) \\ (\gamma-3) / 2 & \left(f_{\mathrm{L}} \gg 1 ; \text { photoelectric }- \text { dominated }\right)\end{array}\right.$

using $T \propto \rho^{\gamma-1}$. If instead $t_{\text {charge }}$ is long $\left(t_{\text {charge }} \gg t_{\mathrm{s}}\right)$, or the grains are at their extremal charge, then $\zeta_{q} \approx 0$.

Note that the grain charge can, in principle, also depend on velocity, which would add a term $-\zeta_{q, w} \mathbf{w}_{\mathrm{s}} \cdot(\delta \mathbf{v}-\delta \mathbf{u}) /\left|\mathbf{w}_{\mathrm{s}}\right|^{2}$ to $\delta t_{L}\left[\mathbf{B}_{0}\right] /\left\langle t_{L}\right\rangle$ (analogous to the velocity dependence in $\zeta_{w}$ ). We could include this, and it does not significantly alter any of our conclusions. However, calculating the velocity dependence of the charge following Shull (1978) (for the collision-dominated case; see their appendix), one obtains $\zeta_{q, w} \approx-0.03 \zeta_{q}$ for $\left|\mathbf{w}_{\mathrm{s}}\right| \lesssim 2 c_{\mathrm{s}}$, and $0<\zeta_{q, w}<0.04 \zeta_{q}$ for all physically relevant $\left|\mathbf{w}_{\mathrm{s}}\right| \gtrsim 2 c_{\mathrm{s}}$. An even weaker (or vanishing) dependence on $\mathbf{v}-\mathbf{u}$ is expected when photo-electric charging dominates and/or when grains have saturated charge. This is therefore a quite small correction in almost all cases, and we neglect it for simplicity.

\subsection{Dispersion relation}

Inserting the Fourier ansatz into equation (4), it takes the form $\omega \mathbf{X}=\mathbb{T} \mathbf{X}$, where $\mathbf{X}=\left(\delta \rho, \delta \rho_{\mathrm{d}}, \ldots\right)$; the full expression is given in Appendix A. The solutions $\omega$ are given by the eigenvalues of $\mathbb{T}$, which is a $10 \times 10$ matrix (the divergence constraint eliminates 1 degree of freedom of the perturbed variables). The general dispersion relation (the characteristic polynomial of $\mathbb{T}$ ) is therefore a 10th-order polynomial, where $\omega$ is a function of 14 independent variables $\left(\mathbf{k}, \hat{\mathbf{B}}_{0} \cdot \hat{\mathbf{w}}_{s}, \zeta_{\mathrm{s}}, \zeta_{w}, \zeta_{q}, w_{\mathrm{s}}, \tau, \beta, \mu, \rho_{0}, c_{\mathrm{s}},\left\langle t_{\mathrm{s}}\right\rangle\right)$.

Although it is straightforward to compute this polynomial from $\mathbb{T}$, it is not in any way intuitive and must be solved numerically. We will therefore focus on exact numerical solutions and simple analytic scalings relevant in certain limits. At any given $\mathbf{k}$ (and fixed fluid+dust properties) there are 10 independent modes (solution branches) for $\omega$. As discussed below, typically $\sim 3-7$ of these are unstable $(\Im(\omega)>0)$. We focus primarily below on the fastest-growing modes (at a given $k$ ), since these will dominate the dynamics.

Figs 2 and 3 plot exact numerical solutions for the growth rate of various unstable modes at a given $k$, as a function of $k$, for fixed values of $\left(\hat{\mathbf{B}}_{0} \cdot \hat{\mathbf{w}}_{\mathrm{s}}, w_{\mathrm{s}}, \tau, \beta, \mu\right)$, and $\left(\zeta_{\mathrm{s}}, \zeta_{w}, \zeta_{q}\right)$ determined according to the cases in Section 3.3. ${ }^{7}$ Figs 4 and 5 plot the growth rates of the fastest growing mode at a given $k$ and similarly fixed equilibrium properties, as a function of the orientation of $\hat{\mathbf{k}}$. We see a very rich mode structure. All of the important features seen here can be understood via appropriate analytic, asymptotic expansions, which we systematically explore in the next several sections.

\footnotetext{
${ }^{7}$ In Fig. 2, for each of the resonant-mode plots, the choice of $\hat{\mathbf{k}}$ is carried out as follows: first, we find the region of mode angles $\theta_{\text {lower }}<\theta_{\mathbf{B k}}<\theta_{\text {upper }}$ where the chosen resonance is possible (considering only $\left.0^{\circ}<\theta_{\mathbf{B k}}<90^{\circ}\right)$, and set $\theta_{\mathbf{B k}}=\left(\theta_{\text {lower }}+\theta_{\text {upper }}\right) / 2$; then, we set the remaining component of $\mathbf{k}$ to satisfy the resonance condition, equation (26). For example, the $\tau=1, \beta=25, \mathbf{w}_{0}=0.1 c_{\mathrm{S}}$ slow-wave resonance is possible for $67^{\circ} \lesssim \theta_{\mathbf{B k}}$; we set $\theta_{\mathbf{B k}}=$ $(67+90) / 2=74.8^{\circ}$, and solve equation (26) to find that $\theta_{\left(\mathbf{w}_{\mathrm{s}} \times \mathbf{B}\right) \mathbf{k}}=-28.6^{\circ}$ is required for the slow-wave resonance. This is not, generally, the fastest growing angle among those that satisfy the resonant condition, but is chosen to be 'typical' (although the growth rate varies weakly within the range of angles that do satisfy the resonant condition, as we show below). In the 'No resonance' case, we arbitrarily choose the mode to propagate at the angle $\theta_{\mathbf{B} \mathbf{~}}=45^{\circ}$ and $\theta_{(\mathbf{w} \times \mathbf{B}) \mathbf{k}}=\cos ^{-1}\left[\mathbf{k} \cdot\left(\mathbf{w}_{\mathrm{s}} \times \mathbf{B}\right)\right]=-45^{\circ}($ see Section 3.4.1).
} 

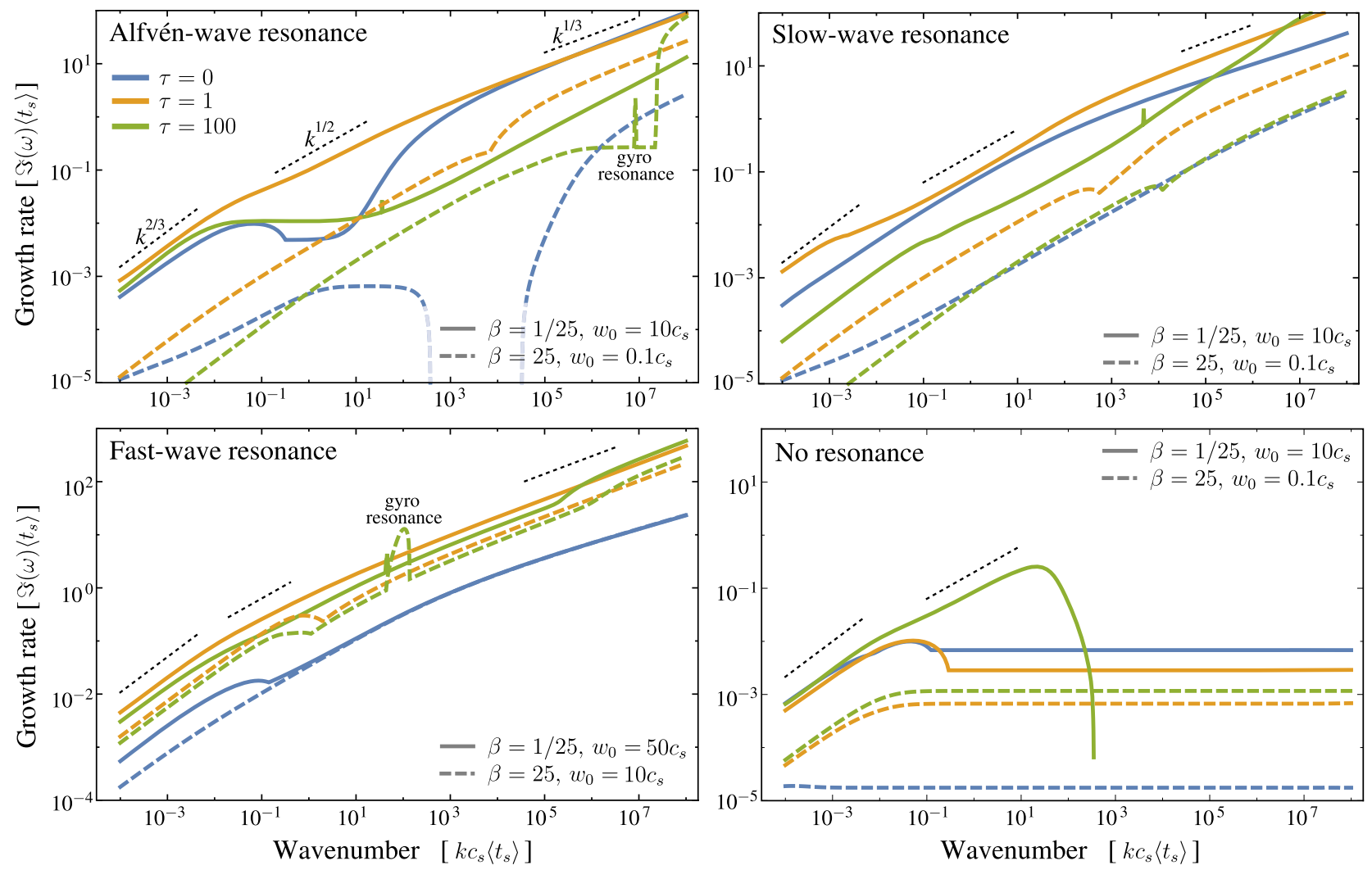

Figure 2. The dispersion relation (growth rate $\Im(\omega)$, in units of $t_{\mathrm{s}}$, as a function of wavenumber $k$ in units of $c_{\mathrm{s}} t_{\mathrm{s}}$ ) of the MHD RDIs for a variety of sample parameters. In the first three panels we choose the mode angle ${ }^{7} \hat{\mathbf{k}}$ such that the mode is in resonance with the Alfvén wave (top-left), the slow magnetosonic wave (top-right), or the fast magnetosonic wave (bottom-left), while at bottom-right we choose $\hat{\mathbf{k}}$ to lie well away from any resonance, for comparison. In each case we plot the dispersion relation at $\beta=1 / 25$ (strong magnetic fields; solid), and $\beta=25$ (weak magnetic fields; dashed), and show $\tau=0$ (unmagnetized grains; blue), $\tau=1$ (intermediate grains; orange), and $\tau=100$ (strongly magnetized grains; green). In all but the fast-wave case, we show both subsonically drifting grains $\left(\left|\mathbf{w}_{0}\right|=0.1 c_{\mathrm{s}}\right)$, and supersonically drifting grains $\left(\left|\mathbf{w}_{0}\right|=10 c_{\mathrm{s}}\right)$, with $\theta_{\mathbf{B a}}=-60^{\circ}$. In the fast-wave case, these parameters eliminate the resonance entirely, so we set $\left|\mathbf{w}_{0}\right|=10 c_{\mathrm{s}}$ and $\left|\mathbf{w}_{0}\right|=50 c_{\mathrm{s}}$, with $\theta_{\mathbf{B a}}=-80^{\circ}$. In all cases we assume Epstein drag in a gas with $\gamma=5 / 3$, and set the dust-to-gas ratio to $\mu=0.01$. We also show line segments (black dotted) illustrating the typical scalings expected for the long-wavelength (low- $k$, Section $\left.4.1 ; \Im(\omega) \propto k^{2 / 3}\right)$, mid-wavelength (Section 5.3; $k^{1 / 2}$ ), and short-wavelength (high- $k$, Section 5.4; $k^{1 / 3}$ ) limits of the MHD-wave RDIs. Some gyro-resonant modes (Section 6 ) are also visible as 'spike' in the dispersion relation when $\tau \gg 1$ (e.g. in the fast-wave dispersion relation at $k c_{\mathrm{s}}\left\langle t_{\mathrm{s}}\right\rangle \sim \tau \sim 100$ ).

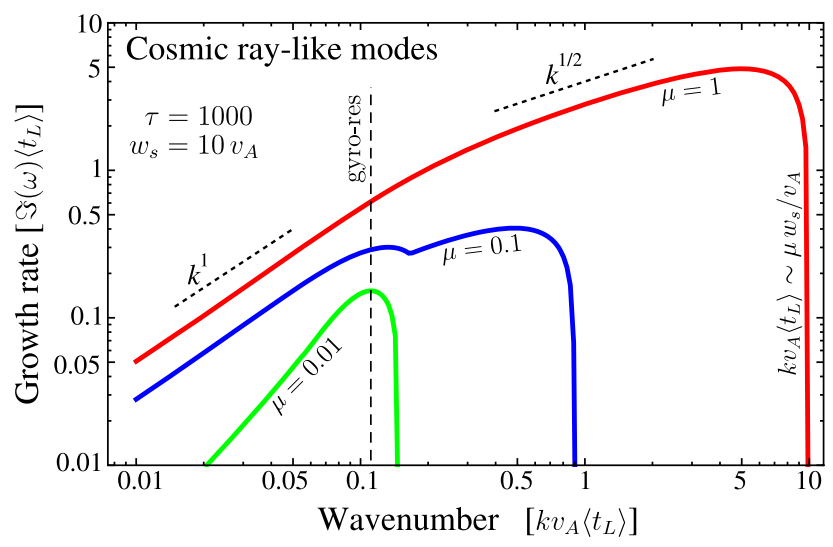

Figure 3. The dispersion relation of the MHD RDI in the high- $\tau$ limit, when it becomes similar to well-known cosmic-ray instabilities (the 'cosmic-ray streaming' modes; see Section 4.3). Note the units of growth rate and wavelength here are the gyro time $t_{\mathrm{L}}$ and $v_{\mathrm{A}} t_{\mathrm{L}}$. We set the streaming aligned with the field, $\theta_{\mathbf{B a}}=0$ (the instability is independent of this choice at sufficiently high $\tau$ ), with $\tau=1000, w_{\mathrm{s}}=10 v_{\mathrm{A}}$, and $c_{\mathrm{S}}=0.1 v_{\mathrm{A}}(\beta=0.01)$. We consider modes aligned with the background drift and magnetic field, $\theta_{\mathbf{a k}}=\theta_{\mathbf{B k}}=0$, since these are generally the fastest growing (Section 4.3), and vary $\mu$ as labeled. When $w_{\mathrm{s}}<v_{\mathrm{A}} \hat{\mu}^{-1 / 2}$ (low $\mu$ or $w_{\mathrm{s}}$; green), only the resonant cosmic-ray instability (Kulsrud \& Pearce 1969) is unstable. This is simply the (parallel) high- $\tau$ limit of the gyro-resonant mode (see Section 6), and is thus strongly peaked around the gyro-resonance (vertical dashed line, where the Larmor-frequency resonates with the Alfvén and fast waves: $k^{-1} \sim w_{\mathrm{S}}\left\langle t_{\mathrm{L}}\right\rangle$ ) and is unstable for $w_{\mathrm{s}}>v_{\mathrm{A}}$. Once $w_{\mathrm{s}} \geq v_{\mathrm{A}} \hat{\mu}^{-1 / 2}$ (blue and red), a new branch of the dispersion relation becomes unstable, which is a manifestation of the non-resonant cosmic-ray instability of Bell (2004) (cf. their Fig. 2), and has larger growth rate at high $\mu$ and/or $w_{\mathrm{s}} / v_{\mathrm{A}}$. The growth rate scales as $\Im(\omega) \sim k\left(\right.$ low- $k$ ) or $\Im(\omega) \sim k^{1 / 2}$ (intermediate- $k$ ), and it is stabilized for $k v_{\mathrm{A}}\left\langle t_{\mathrm{L}}\right\rangle \gtrsim \mu w_{\mathrm{s}} / v_{\mathrm{A}}$. 

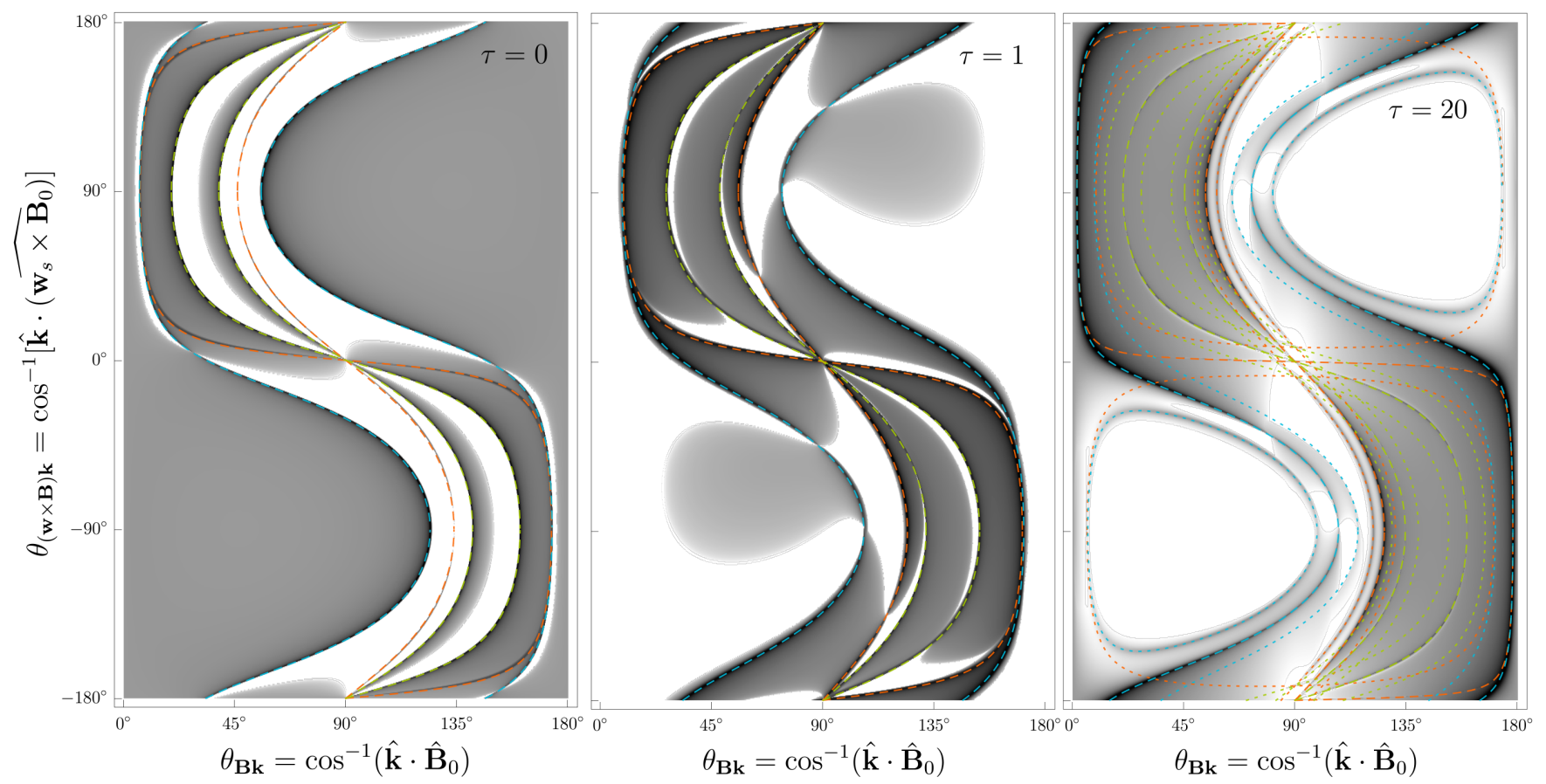

Figure 4. The angular structure of the MHD RDIs, showing how the fastest-growing modes appear at the resonant angles. Shading in each panel shows the growth rate (increasing logarithmically from $\Im(\omega)\left\langle t_{\mathrm{s}}\right\rangle<10^{-4}$ [light] to $>1$ [dark]), evaluated at $k=|\mathbf{k}|=50 /\left(c_{\mathrm{s}}\left\langle t_{\mathrm{s}}\right\rangle\right)$. The mode angle $\hat{\mathbf{k}}$ is parametrized by the angles $\theta_{\mathbf{B k}}=\cos ^{-1}\left(\hat{\mathbf{k}} \cdot \hat{\mathbf{B}}_{0}\right)$ and $\theta_{(\mathbf{w} \times \mathbf{B}) \mathbf{k}}=\cos ^{-1}\left(\hat{\mathbf{k}} \cdot \widehat{\mathbf{w}_{\mathbf{s}} \times \mathbf{B}_{0}}\right)$ (see Section 3.4.1). Colored lines denote the angles that satisfy the 'resonance condition' (equation 26), for the Alfvén (red), slow (green), and fast (blue) wave-RDI resonances (dashed; equation 26) or gyro-RDI resonances (dotted; equation 43, only present when $\tau>1$ ). Left: Solutions for $\tau=0$, with $\left|\mathbf{w}_{0}\right|=5 c_{\mathrm{s}}, \theta_{\mathbf{B a}}=-60^{\circ}, \beta=1 / 4$, dust-to-gas ratio $\mu=0.01$, and Epstein drag with $\gamma=5 / 3$. Middle: Same parameters as left, except $\tau=1$. Right: Solutions for $\tau=20$, with $\left|\mathbf{w}_{0}\right|=60 c_{s}, \theta_{\mathbf{B a}}=-88^{\circ}, \beta=1 / 4, \mu=10^{-5}$, Epstein drag with $\gamma=5 / 3$ (we change parameters so the resonances are more visually well-separated). The fastest-growing modes invariably occur at the predicted (resonant or gyro-resonant) mode angles, although in the $\tau=20$ case, there are certain gyro-resonances not associated with a fast-growing mode, as expected (see discussion in Section 6.2).

\subsubsection{Parametrization of $\hat{\mathbf{k}}$}

It is worth briefly commenting on our parametrization of the mode direction $\hat{\mathbf{k}}$. While for analytic results (see Section 5.2), it is most convenient to use a Cartesian coordinate system for $\mathbf{k}$, a polar system is more convenient for plotting, because $k=|\mathbf{k}|$ is naturally kept fixed. Thus in Figs 2 and 5, we parametrize $\hat{\mathbf{k}}$ through its angle from $\hat{\mathbf{B}}_{0}$,

$\theta_{\mathbf{B k}}=\cos ^{-1}\left(\hat{\mathbf{k}} \cdot \hat{\mathbf{B}}_{0}\right)$,

and the angle subtended from $\hat{\mathbf{y}}=\widehat{\mathbf{w}_{\mathrm{s}} \times \mathbf{B}_{0}}$,

$\theta_{(\mathbf{w} \times \mathbf{B}) \mathbf{k}}=\cos ^{-1}\left(\hat{\mathbf{k}} \cdot \widehat{\mathbf{w}_{\mathrm{s}} \times \mathbf{B}_{0}}\right)$,

which is simply the standard azimuthal angle in spherical polar coordinates about $\hat{\mathbf{B}}_{0}$ (shifted by $90^{\circ}$ ). While this parametrization is arbitrary, it has the advantage of making the resonant lines more obvious (e.g. in Fig. 4).

\section{PARALlel/ALIGNED MODES: THE PRESSURE-FREE, COSMIC-RAY STREAMING, AND ACOUSTIC (QUASI-DRIFT AND QUASI-SOUND) MODES}

We first consider the 'parallel' modes from Section 2, where the behavior of greatest interest (e.g. fastest growth rates) occurs when $\hat{\mathbf{k}} \approx \mathbf{w}_{\mathrm{s}}$, as compared to the more complicated angle-dependent resonances we will discuss in subsequent sections.

\subsection{The long-wavelength (low- $k$ ) or 'pressure free' mode}

At sufficiently low- $k$, the structure of the fastest-growing unstable mode is actually rather simple (and instructive). For the acoustic RDI (neutral gas and neutral grains; Paper II), we showed that at low- $k$, the fastest-growing mode satisfies $\omega=\omega_{\mathrm{PF}} \sim \mathcal{O}\left(k^{2 / 3}\right)$; this is true here as well. Expanding the dispersion relation in powers of $k /\left(c_{\mathrm{s}}\left\langle t_{\mathrm{s}}\right\rangle\right) \ll \hat{\mu}$, we obtain

$\omega_{\mathrm{PF}}^{3}=i \mathcal{F}_{\mathrm{PF}} \frac{\hat{\mu} w_{\mathrm{s}}^{2} k^{2}}{\left\langle t_{\mathrm{s}}\right\rangle}+\mathcal{O}\left(k^{4}\right)$. 

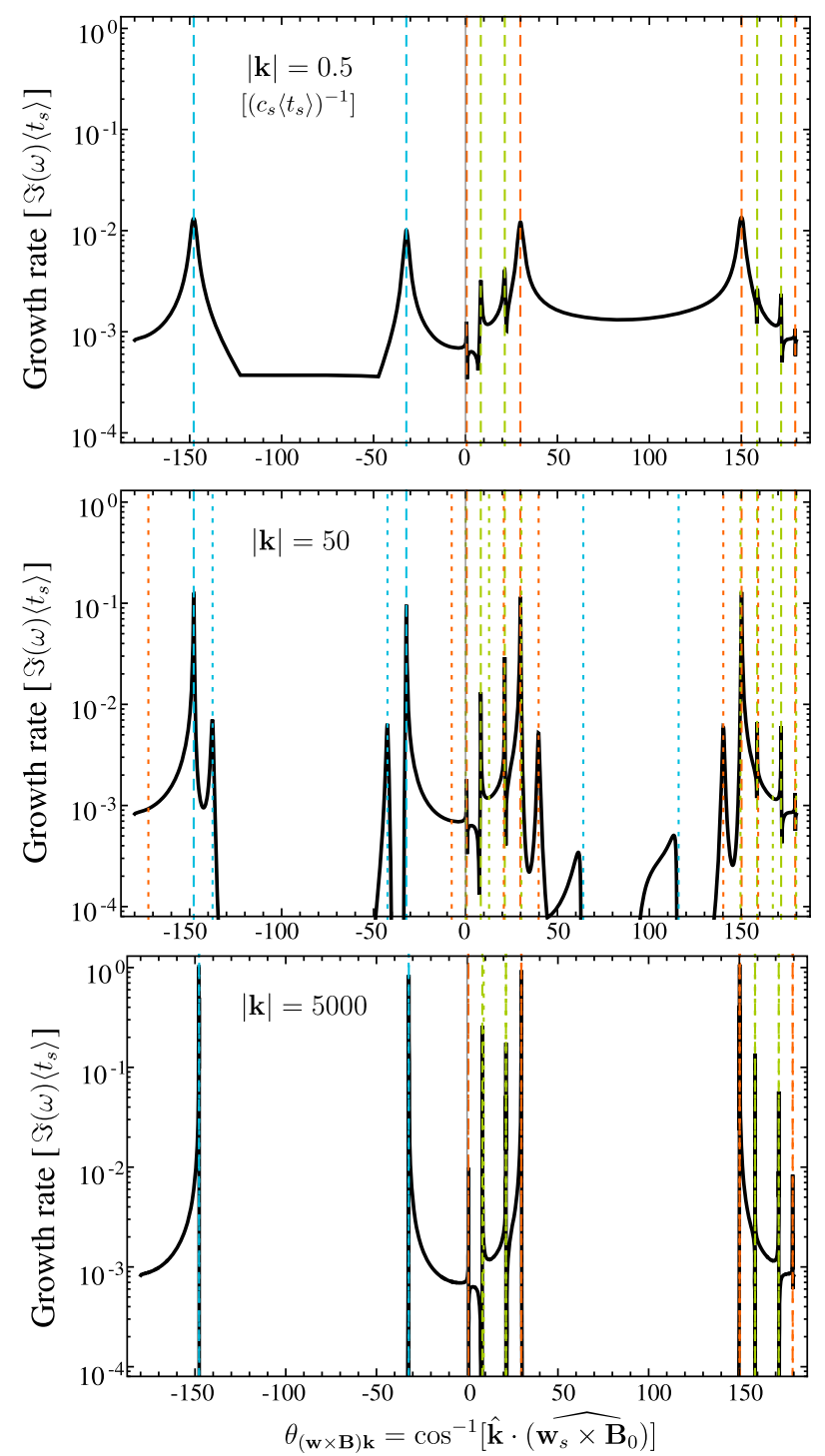

Figure 5. $1 \mathrm{D}$ angular structure of the MHD RDIs, taking a vertical 'slice' through Fig. 4; i.e. we fix the angle between $\hat{\mathbf{k}}_{\text {and }} \hat{\mathbf{B}}_{0}$ at $\theta_{\mathbf{B k}}=70^{\circ}$, and plot the growth rate $\Im(\omega)$ versus the varied free angle $\theta_{(\mathbf{w} \times \mathbf{B}) \mathbf{k}}$. Parameters are fixed to those used in the $\tau=20$ case in Fig. $4\left(\left|\mathbf{w}_{0}\right|=60 c_{s}, \theta_{\mathbf{B a}}=-88^{\circ}, \beta=1 / 4\right.$, $\left.\mu=10^{-5}\right)$, but we consider three wavenumbers: $k=|\mathbf{k}|=0.5 /\left(c_{\mathrm{s}}\left\langle t_{\mathrm{s}}\right\rangle\right)($ top $), k=50 /\left(c_{\mathrm{s}}\left\langle t_{\mathrm{s}}\right\rangle\right)($ middle $), k=5000 /\left(c_{\mathrm{s}}\left\langle t_{\mathrm{s}}\right\rangle\right)($ bottom). Colored lines show the resonant angles as in Fig. 4: Alfén (red), slow (green), and fast (blue) MHD-wave RDIs (dashed; equation 26) or gyro-RDIs (dotted; equation 43). Note that $k=0.5$ (top) is too low- $k$ for any gyro-resonant condition to be met, while the gyro-resonances become indistinguishable from the MHD-wave RDI resonances for the high- $k=5000$ case (bottom). For intermediate- $k=50$ (middle), we see that the fast-wave gyro-resonances are strongest, while some of the Alfvén-wave gyro-resonances and all of the slow-wave gyro-resonances are too weak to be visible at these parameters (see Section 6).

where $\mathcal{F}_{\mathrm{PF}}$ is a real scalar ${ }^{8}$ that depends on the drift law, relative strength of Lorentz forces $(\tau)$, and angles between $\hat{\mathbf{k}}$, $\hat{\mathbf{w}}_{\mathrm{s}}$, and $\hat{\mathbf{B}}_{0}$. The expression for $\mathcal{F}_{\mathrm{PF}}$ is complicated, but for $\zeta_{\mathrm{s}}=\zeta_{w}=\zeta_{q}=0$ (constant $t_{\mathrm{s}}$ and $t_{\mathrm{L}}$ ) it simplifies to

$\mathcal{F}_{\mathrm{PF}}\left(\zeta_{s}, \zeta_{w}, \zeta_{q}=0\right) \rightarrow\left(\hat{\mathbf{k}} \cdot \hat{\mathbf{w}}_{\mathrm{s}}\right)^{2}-\frac{\left[1+\left(\tau \hat{\mathbf{k}} \cdot \hat{\mathbf{B}}_{0}\right)^{2}\right] \sin ^{2} \theta_{\mathbf{B w}}}{1+\tau^{-2}}$

${ }^{8}$ The full expression for $\mathcal{F}_{\mathrm{PF}}$ in equation (16) is

$$
\begin{aligned}
\mathcal{F}_{\mathrm{PF}} & =\left[1+\left(c_{0}^{2}+\tilde{\zeta}_{w}^{-1} s_{0}^{2}\right) \tau^{2}\right]^{-1}\left[\hat{k}_{z}^{2}\left(1-\zeta_{\mathrm{s} / w}\right)-s_{0} \hat{k}_{y} \hat{k}_{z} \zeta_{q} \tau-f_{2} \tau^{2}-f_{3} \tau^{3}-\tilde{\zeta}_{w}^{-1} s_{0}^{2}\left(\hat{\mathbf{k}} \cdot \hat{\mathbf{B}}_{0}\right)^{2} \tau^{4}\right], \\
f_{2} & =s_{0}^{2}\left[1+\hat{k}_{z}^{2}\left(\zeta_{q / w}-1\right)+\hat{k}_{y}^{2}\left(\zeta_{q}-\zeta_{\mathrm{s} / w}\right)\right]-c_{0}^{2} \hat{k}_{z}^{2}\left(1-\zeta_{\mathrm{s} / w}\right)-s_{0} c_{0} \hat{k}_{x} \hat{k}_{z}\left(\zeta_{q}-\zeta_{\mathrm{s} / w}\right), \\
f_{3} & =s_{0} \hat{k}_{y}\left[\hat{k}_{z}\left(s_{0}^{2} \zeta_{q / w}+c_{0}^{2} \zeta_{\mathrm{s} / w}\right)+s_{0} c_{0} \hat{k}_{x}\left(\zeta_{\mathrm{s} / w}-\zeta_{q}\right)\right] .
\end{aligned}
$$




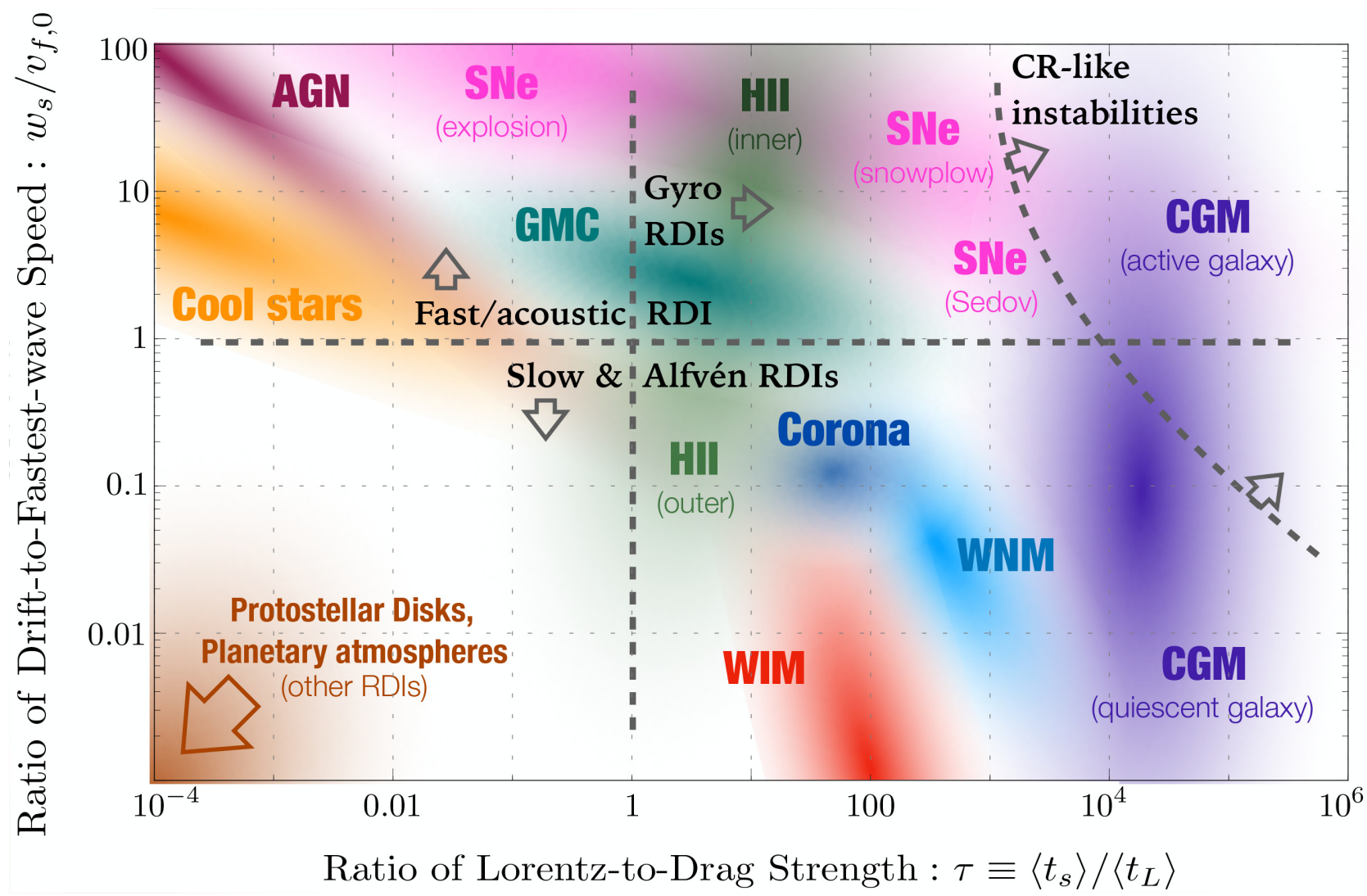

Figure 6. Illustration of the two most important dimensionless parameters of the MHD RDI - the dust drift speed normalized by the fast wave speed, $w_{\mathrm{s}} / v_{\mathrm{f}, 0}$, and the ratio of stopping time to Larmor time, $\tau \equiv\left\langle t_{\mathrm{s}}\right\rangle /\left\langle t_{\mathrm{L}}\right\rangle$ - for a variety of different astrophysical environments, as discussed in the text (Section 9 ). As labeled on the figure, the different shaded regions illustrate: the warm-neutral medium (WNM, light blue; Section 9.2.1); the warm-ionized medium (WIM, red; Section 9.2.1); the circum-galactic medium (CGM) and/or inter-galactic medium (CGM, purple; Section 9.2.2, around both quiescent or active/quasar-hosting galaxies); H II regions both near and far from the ionizing radiation source (H II , dark green; Section 9.2.3); supernovae in various phases of evolution (SNe, pink; Section 9.2.4); Solar coronal dust (blue; Section 9.2.5); cool star photospheres and winds (orange; Section 9.2.6); giant molecular clouds and star-forming regions (GMC, sea green; Section 9.2.7); active-galactic nucleii (AGNs, maroon; see Paper II and Section 9.2.7); and proto-stellar/planetary discs/atmospheres (brown; Section 9.2.8), which extend off the plotted range (as labeled). We also illustrate, with the gray dotted lines and arrows, where different forms of the RDI should be unstable; the fast (acoustic) RDI is unstable for $w_{\mathrm{s}} / v_{\mathrm{f}, 0} \gtrsim 1$, the gyro-resonant RDIs can be unstable for $\tau \gtrsim 1$, and the cosmic-ray like instabilities (Section 4.3) may dominate for very large $\tau$.

The dispersion relation has the form $\omega_{\mathrm{PF}}^{3}=X$; this always has an unstable $\left(\Im\left(\omega_{\mathrm{PF}}\right)>0\right)$ root, for any complex $X$ with $\|X\| \neq 0$. If we write $X=\|X\| \exp \left(i \phi_{X}\right)$ then we can define $\omega_{\mathrm{PF}}=i_{0}\|X\|^{1 / 3}$ where $i_{0}$ is the root of $i_{0}^{3}=\exp \left(i \phi_{X}\right)$ (so $\left.\left\|i_{0}\right\|=1\right)$ with the largest imaginary part (such that $\Im\left(\omega_{\mathrm{PF}}\right)>0$ ). For the example above, since $\mathcal{F}_{\mathrm{PF}}$ is purely real, we see that $i_{0}=i$ if $\mathcal{F}_{\mathrm{PF}}<0$ and $i_{0}=(i \pm \sqrt{3}) / 2$ if $\mathcal{F}_{\mathrm{PF}}>0$.

Note that in equation (18), if we naively took $\tau \rightarrow \infty, \mathcal{F}_{\mathrm{PF}}$ would appear to diverge as $\tau^{2}$. However, recall from Section 3.1 that as $\tau \rightarrow$ $\infty$, Lorentz forces project the drift direction $\left(\hat{\mathbf{w}}_{\mathrm{s}}\right)$ onto the field direction $\left(\hat{\mathbf{B}}_{0}\right)$, so $\sin \theta_{\mathbf{B w}} \rightarrow(1 / \tau) \tan \theta_{\mathbf{B a}}$. Recall that $\theta_{\mathbf{B a}}$ here is the angle between the field $\left(\hat{\mathbf{B}}_{0}\right)$ and whatever acceleration a is sourcing the drift (or equivalently, the direction $\hat{\mathbf{w}}_{0}=\hat{\mathbf{a}}$ which the drift would have without Lorentz forces). So for a fixed external acceleration $\mathbf{a}$ ( or $\left.\mathbf{w}_{0}\right), \mathcal{F}_{\mathrm{PF}}$ remains finite.

Thus, considering the low and high- $\tau$ limits and writing expressions in terms of $\mathbf{w}_{0}$ (rather than $\mathbf{w}_{\mathrm{s}}$ ), we can express $\omega_{\mathrm{PF}}$ as

$$
\begin{aligned}
\omega_{\mathrm{PF}} & =i_{0}\left|\frac{f_{1}}{\tilde{\zeta}_{w}} \frac{\hat{\mu} w_{0}^{2}\left(\hat{\mathbf{w}}_{\mathrm{s}} \cdot \mathbf{k}\right)^{2}}{\left\langle t_{\mathrm{s}}\right\rangle}\right|^{1 / 3}, \\
f_{1} & \equiv \begin{cases}\left(\tilde{\zeta}_{w}-\zeta_{\mathrm{s}}\right) & (\tau \ll 1) \\
\left(\tilde{\zeta}_{w}-\zeta_{\mathrm{s}}\right) \cos ^{2} \theta_{\mathbf{B a}}-\zeta_{\mathrm{s}} \frac{\hat{k}_{y}}{\hat{k}_{z}} \cos \theta_{\mathbf{B a}} \sin \theta_{\mathbf{B a}}-\sin ^{2} \theta_{\mathbf{B a}} & (\tau \gg 1)\end{cases}
\end{aligned}
$$

Here $f_{1}$ has the same sign as $\mathcal{F}_{\mathrm{PF}}$ so $i_{0}=i$ if $f_{1}<0$ and $i_{0}=(i \pm \sqrt{3}) / 2$ if $f_{1}>0$.

We immediately see that the growth rates scale as $\Im(\omega) \sim\left(\hat{\mu} w_{\mathrm{s}}^{2} k^{2} /\left\langle t_{\mathrm{s}}\right\rangle\right)^{1 / 3}$, modulo order-unity geometric corrections, and the fastestgrowing modes have $\mathbf{k}$ aligned with the drift $\hat{\mathbf{w}}_{\mathrm{s}}$. We can see in Fig. 2 that this provides an excellent approximation to the scalings of exact solutions for $k c_{\mathrm{s}}\left\langle t_{\mathrm{s}}\right\rangle \ll \hat{\mu}$. For $\tau \ll 1$, the scaling here is identical to the low- $k$ mode of the acoustic RDI from Paper II. As discussed there, this is because this mode dominates at sufficiently low $k$ such that the pressure forces (which scale as $\nabla P \propto k$ ) become much smaller than the drag force between dust and gas $(\propto \mu)$. The same is true of magnetic pressure, so the result is independent of $\beta$. For large $\tau$, the correction is essentially geometric, from the projection of the drift (by Lorentz forces) onto directions not aligned with the 'forcing' term a. Also note 
that although the growth rate for $\tau \ll 1$ appears to vanish when $\zeta_{s}=\tilde{\zeta}_{w}$, an instability is still in fact present (but the leading-order term in our series expansion vanishes; see Paper II); for $\tau \gg 1$ this does not significantly alter the growth rate because of the other terms that do not depend on $\tilde{\zeta}_{w}-\zeta_{s}$.

Because the relevant wavelengths of this mode are larger than the 'stopping length' $\lambda_{\text {stop }} \sim w_{\mathrm{s}}\left\langle t_{\mathrm{s}}\right\rangle$ of the grains, and sufficiently large that perturbed pressure and MHD effects are weak, the mode structure is quite simple and essentially the same as in un-magnetized fluids (see Section 3.9 of Paper II for details). The perturbation is a longitudinal ( $\delta \mathbf{v} \propto \delta \mathbf{u} \propto \mathbf{k} \propto \mathbf{w}_{\mathrm{s}}$ ), compressible perturbation of the joint dust-and-gas fluid, which features dust and gas fluctuations nearly in-phase with one another $\left(\delta \mathbf{v} \sim \delta \mathbf{u}, \delta \rho_{\mathrm{d}} \sim \mu \delta \rho\right.$, but the velocity and density fluctuations are out-of-phase by $\left.\sim 30^{\circ}\right)$, with a phase velocity $\mathbf{w}_{\mathrm{s}}\left(k w_{\mathrm{s}}\left\langle t_{\mathrm{s}}\right\rangle / \mu\right)^{1 / 3}$. As a result the dust-to-gas fluctuations driven by this mode are smaller than the absolute density fluctuation: however, they accumulate dust in the form of enhanced 'sheets' of dust overdensity in the plane perpendicular to the drift direction (and moving with the drift).

\subsection{The strongly Lorentz-dominated limit: the acoustic (quasi-sound and quasi-drift) modes}

Under many conditions, at intermediate and high- $k$, the resonant modes described below are the fastest-growing. However, just like the non-resonant low- $k$ modes in Section 4.1, it is instructive to consider the strongly Lorentz-dominated case, where other modes may in fact be the fastest-growing. Specifically, here we refer to the case when $\tau \gg 1$ is sufficiently large that $\tau$ is much larger than any other parameter in the problem (including higher powers, e.g. $\tau \gtrsim k^{3}$ is required for the expansions below to be formally valid), and $1 / \tau$ is sufficiently small that it is also an expansion parameter (e.g. $1 / \tau \ll \mu, 1 / \tau \ll k$ ).

In this limit, for fixed external acceleration $\mathbf{a}\left(\right.$ or $\mathbf{w}_{0}$ ), the drift velocity $\mathbf{w}_{\mathrm{s}}$ becomes aligned with the magnetic field, $\hat{\mathbf{w}}_{\mathrm{s}} \approx \hat{\mathbf{B}}_{0}$. The resonant modes, which mostly require $\hat{\mathbf{k}}$ to have large components perpendicular to $\hat{\mathbf{B}}$, can be suppressed. For example, we will show below that the growth rates of the mid- $k$ Alfvén and slow RDIs can be suppressed at large $\tau$, which, physically, is related to the Lorentz forces suppressing motion perpendicular to the field lines. While the high- $k$ Alfvén mode is not suppressed by large $\tau$, it may not appear until extremely large $k$ when $\tau$ is large. So in this limit the fastest-growing modes will often be the parallel modes, with $\hat{\mathbf{k}} \approx \hat{\mathbf{w}}_{\mathrm{s}} \approx \hat{\mathbf{B}}_{0}$. If we assume this ${ }^{9}$ and expand the dispersion relation in $\tau^{-1}$, we obtain two interesting branches of the dispersion relation.

One branch is identical to the dispersion relation for parallel modes $\left(\hat{\mathbf{k}}=\hat{\mathbf{w}}_{\mathrm{s}}\right)$ in the non-magnetized acoustic case studied in Paper II. ${ }^{10}$ Because they are longitudinal, parallel modes, the gas responds to the dust just like the pure hydrodynamic case (neither $\tau$ nor $\beta$ appears in the dispersion relation). From Paper II, we know this has two unstable modes, the 'quasi-drift' $\left(\omega_{\mathrm{QD}}\right)$ and 'quasi-sound' ( $\left.\omega_{\mathrm{QS}}\right)$ modes. The details are given in Paper II, but we summarize them here. Both are longitudinal and parallel and field-aligned in this limit $\left(\delta \mathbf{v} \propto \delta \mathbf{u} \propto \mathbf{k} \propto \mathbf{w}_{\mathrm{s}} \propto \mathbf{B}_{0}\right)$. In both cases the mode resembles a sound wave in the gas, but the dust velocity and density fluctuations are out-of-phase both with the gas and with each other, with the dust density fluctuation lagging the gas by a phase offset $\sim 30^{\circ}$. This in turn generates a very large dust response, with $\mu^{-1}\left|\delta \rho_{\mathrm{d}} / \delta \rho\right| \gg 1$ at high- $k$.

The 'quasi-drift' mode is a modified dust-drift mode with frequency (at high $k$ ) given approximately by (derived in Paper II):

$\omega_{\mathrm{QD}} \approx k w_{\mathrm{s}}+i \frac{\tilde{\zeta}_{w}}{2\left\langle t_{\mathrm{s}}\right\rangle}\left[-1+\left(1+\frac{4 \mu\left(\tilde{\zeta}_{w}-\zeta_{\mathrm{s}}\right)}{\tilde{\zeta}_{w}^{2}\left(1-c_{\mathrm{s}}^{2} / w_{\mathrm{s}}^{2}\right)}\right)^{1 / 2}\right]$,

i.e. phase velocity $\sim \mathbf{w}_{\mathrm{s}}$ and growth rate $\Im\left(\omega_{\mathrm{QD}}\right) \sim\left(\mu /\left\langle t_{\mathrm{s}}\right\rangle\right)\left[\left(1-\zeta_{s / w}\right) /\left(1-c_{\mathrm{s}}^{2} / w_{\mathrm{s}}^{2}\right)\right]$. Thus, the mode is typically unstable if $w_{\mathrm{s}} \geq c_{\mathrm{s}}($ given that Epstein drag dominates when $w_{\mathrm{s}}>c_{\mathrm{s}}$, and has $\zeta_{\mathrm{s} / w}<1$ for essentially all physical gas equations-of-state), or if $w_{\mathrm{s}} \leq c_{\mathrm{s}}$ and Coulomb drag dominates (which then gives $\zeta_{\mathrm{s} / w}>1$ for equations-of-state $\gamma \gtrsim 1$ ).

The 'quasi-sound' mode is a modified sound wave with

$\omega_{\mathrm{QS}} \approx \pm c_{\mathrm{s}} k+i \frac{\mu}{2\left\langle t_{\mathrm{s}}\right\rangle}\left[ \pm \frac{w_{\mathrm{s}}}{c_{\mathrm{S}}}\left(\zeta_{\mathrm{s}}-1\right)-\tilde{\zeta}_{w}\right]$

i.e. phase velocity $\sim c_{\mathrm{s}} \hat{\mathbf{k}}$, and growth rate $\Im\left(\omega_{\mathrm{QS}}\right) \sim\left(\mu / 2\left\langle t_{\mathrm{s}}\right\rangle\right)\left(w_{s}\left|1-\zeta_{\mathrm{s}}\right| / c_{\mathrm{s}}-\tilde{\zeta}_{w}\right)$. Thus, the mode is unstable when $w_{\mathrm{s}} / c_{\mathrm{s}} \gtrsim \tilde{\zeta}_{w} /\left|1-\zeta_{\mathrm{s}}\right| \sim 1$ (i.e. typically when the drift is super-sonic).

In both cases, the growth rates are essentially independent of $k$ : they are the 'out of resonance' versions of the acoustic RDI, or more precisely, the fast magnetosonic RDI (the mode structure is discussed in detail in Paper II). However, under these high- $\tau$ conditions, they can be faster-growing than the resonant modes, because of the suppression of modes perpendicular to $\hat{\mathbf{B}}_{0}$. At high- $k$ and high- $\tau$, these are slowergrowing than the 'cosmic ray'-like modes described below; however, at low- $k$, (or at some intermediate- $\tau$ ) these can be the fastest-growing modes (because their growth rate does not depend on $k$ ).

\footnotetext{
${ }^{9}$ More rigorously, we can use the full expression for $\mathbf{w}_{\mathbf{s}}$ as a function of $\mathbf{w}_{0}$ and $\tau$ in equation (3) to obtain the dispersion relation, expand in $\tau^{-1}$, then marginalize over angles $\hat{\mathbf{k}}$, but this gives the same expressions.

${ }^{10}$ Specifically, $\quad 0=c_{\mathrm{s}} k \mu\left(\omega \tilde{\zeta}_{w}-k w_{\mathrm{s}} \zeta_{\mathrm{s}}\right)+\left\langle t_{\mathrm{s}}\right\rangle^{2}\left(\omega-k w_{\mathrm{s}}\right)\left[\left(\omega-k w_{\mathrm{s}}+i \tilde{\zeta}_{w} /\left\langle t_{\mathrm{s}}\right\rangle\right)\left(\omega^{2}-c_{\mathrm{s}}^{2} k^{2}\right)+i \mu\left\langle t_{\mathrm{s}}\right\rangle^{-1}\left(\omega^{2} \tilde{\zeta}_{w}+w_{\mathrm{s}} k\left\{w_{\mathrm{s}} k\left(\zeta_{\mathrm{s}}-1\right)+i \tilde{\zeta}_{w} /\left\langle t_{\mathrm{s}}\right\rangle\right\}-\right.\right.$ $\left.\left.\omega w_{\mathrm{s}} k\left\{\tilde{\zeta}_{w}+\zeta_{\mathrm{s}}-1\right\}\right)\right]$, which is the dispersion relation for $\hat{\mathbf{k}}=\hat{\mathbf{w}}_{\mathrm{s}}$ from Paper II after factoring out the un-interesting purely damped modes.
} 


\subsection{The strongly Lorentz-dominated limit: the 'cosmic ray' modes}

In the strongly Lorentz-force-dominated limit (very large- $\tau$ ), with $\hat{\mathbf{w}}_{\mathrm{s}} \approx \hat{\mathbf{B}}_{0}$, there are two other interesting branches of the dispersion relation for parallel modes $\left(\hat{\mathbf{k}} \approx \hat{\mathbf{w}}_{\mathrm{s}} \approx \hat{\mathbf{B}}_{0}\right.$ ). These modes are the manifestation of well-known cosmic-ray instabilities (Kulsrud \& Pearce 1969; Bell 2004), and thus also remain unstable in the absence of drag forces (i.e. if $t_{\mathrm{s}}=0$ ). In fact, because their growth rates are set by $\left\langle t_{\mathrm{L}}\right\rangle$ (as opposed to $\left\langle t_{\mathrm{s}}\right\rangle$ ), for $\tau \gg 1$, these modes can grow much faster than the stopping time, which is the time-scale required for the system to reach equilibrium (see e.g. Fig. 1). Thus the results of this section are somewhat qualitative, and a more complete treatment would allow for arbitrary dust distribution functions (rather than assuming the dust to be pressure-less fluid, as done here). Many such treatments exist in the cosmic-ray literature (see references below).

The first of the cosmic-ray modes is simply the high- $\tau$ limit of the gyro-resonance mode. This will be discussed in detail in Section 6 , and so here we simply note that it is related to the resonant cosmic-ray instability (Kulsrud \& Pearce 1969; Wentzel 1969; McKenzie \& Voelk 1982), arising through the resonant interaction between an MHD wave and the dust/cosmic-ray gyro-motion. ${ }^{11}$ The resonance with the Alfvén wave generally leads to the fastest-growing mode, and is unstable for $w_{\mathrm{s}}>v_{\mathrm{A}}$.

The other cosmic-ray instability is a manifestation ${ }^{12}$ of the non-resonant instability of Bell (2004). These modes are fastest-growing in the parallel direction $\left(\hat{\mathbf{k}} \approx \hat{\mathbf{w}}_{\mathrm{s}} \approx \hat{\mathbf{B}}_{0}\right.$ ), and only weakly affected by the fluid pressure (i.e. $\beta$ ) because they involve interactions between the dust and Alfvén waves. As described above (Section 4.2) for the acoustic modes, we may derive their dispersion relation through an expansion in $\tau \gg 1$, with $\hat{\mathbf{k}} \approx \hat{\mathbf{w}}_{\mathrm{s}} \approx \hat{\mathbf{B}}_{0}$. In this limit, the full dispersion relation factors into a product of various terms and $k_{z}^{2}\left[v_{\mathrm{A}}^{2}\left(w_{\mathrm{s}} k_{z}\left\langle t_{\mathrm{L}}\right\rangle+1\right)-\mu w_{\mathrm{s}}^{2}\right]+$ $\omega k_{z}\left(2 \mu w_{\mathrm{s}}-v_{\mathrm{A}}^{2} k_{z}\left\langle t_{\mathrm{L}}\right\rangle\right)+\omega^{2}\left(-w_{\mathrm{s}} k_{z}\left\langle t_{\mathrm{L}}\right\rangle-\mu-1\right)+\omega^{3}\left\langle t_{\mathrm{L}}\right\rangle$, which, set to zero, gives the dispersion relation of the non-resonant cosmic-ray mode.

As usual, we are particularly interested in the roots where $\omega$ has a positive imaginary component, signaling an unstable mode. As in Bell (2004), one finds three regimes for the solutions $\omega\left(k_{z}\right)$ depending on the wavelength. At longer wavelengths, $k v_{\mathrm{A}}\left\langle t_{\mathrm{L}}\right\rangle \lesssim(1+\mu) v_{\mathrm{A}} / w_{\mathrm{s}}$, an expansion in $k v_{\mathrm{A}}\left\langle t_{\mathrm{L}}\right\rangle$ yields the solution

$\omega=\omega_{\mathrm{Str}} \approx k w_{\mathrm{s}} \hat{\mu}+i k v_{\mathrm{A}}\left[\frac{\left(w_{\mathrm{s}} / v_{\mathrm{A}}\right)^{2} \hat{\mu}-1}{1+\mu}\right]^{1 / 2}$,

while for shorter wavelengths, $(1+\mu) v_{\mathrm{A}} / w_{\mathrm{s}} \lesssim k v_{\mathrm{A}}\left\langle t_{\mathrm{L}}\right\rangle$, an expansion in $w_{\mathrm{s}} / v_{\mathrm{A}} \gg 1$ yields

$\omega=\omega_{\mathrm{Str}} \approx \frac{\mu}{\left\langle t_{\mathrm{L}}\right\rangle}+i\left[\frac{\mu w_{\mathrm{s}}}{\left\langle t_{\mathrm{L}}\right\rangle} k_{z}-\left(k_{z} v_{\mathrm{A}}\right)^{2}-\frac{\mu^{2}}{\left\langle t_{\mathrm{L}}\right\rangle^{2}}\right]^{1 / 2}$,

which scales as $\Im(\omega) \sim\left(\mu w_{\mathrm{s}}\left\langle t_{\mathrm{L}}\right\rangle\right)^{1 / 2} k_{z}^{1 / 2}$ for $k v_{\mathrm{A}}\left\langle t_{\mathrm{L}}\right\rangle \lesssim \mu w_{\mathrm{s}} / v_{\mathrm{A}}$ but is stabilized at the shortest wavelengths, $k v_{\mathrm{A}}\left\langle t_{\mathrm{L}}\right\rangle \gtrsim \mu w_{\mathrm{s}} / v_{\mathrm{A}}$. It transpires that the condition for this non-resonant mode to be unstable (across all wavelengths) is given correctly by equation $(22)$ as $\left(w_{\mathrm{s}} / v_{\mathrm{A}}\right)^{2} \hat{\mu}>1$, i.e. $w_{\mathrm{s}}>v_{\mathrm{A}} \hat{\mu}^{-1 / 2}$. This condition can be satisfied relatively easily in many systems (Bell 2004; Riquelme \& Spitkovsky 2009), particularly at high $\beta$. It is also worth recalling that because $\left\langle t_{\mathrm{L}}\right\rangle=\left\langle t_{\mathrm{s}}\right\rangle / \tau$ and $\tau \gg 1$, this instability has very short wavelengths and fast growth rates when considered in the units of the drag time (e.g. above and in Paper II). Thus it can grow much faster than the drag-induced quasi-sound and quasi-drift modes discussed in Section 4.2.

Considering the eigenvectors of the linear mode in detail shows that the mode resembles a mix of Alfvén waves, with $w_{\mathrm{s}}$ playing the role of the phase velocity $v_{\mathrm{A}}$ when $w_{\mathrm{s}} \gg v_{\mathrm{A}} \hat{\mu}^{-1 / 2}$. Specifically, the perturbation is very weakly compressible, with the longitudinal components of $\delta \mathbf{u}, \delta \mathbf{v}$ and corresponding density perturbations present but suppressed by large powers of $\mu$ and $k$. Instead it is primarily transverse, featuring dust executing gyro-motion with coupled perpendicular perturbations of the field $\delta \mathbf{B}_{x, y} \approx\left(\delta \mathbf{v}_{x, y}-\delta \mathbf{u}_{x, y}\right) / w_{\mathrm{s}}$ (akin to a super-position of a forward-propagating Alfvén wave in the gas and backward-propagating Aflvén wave in dust, with $v_{\mathrm{A}}$ replaced by $w_{\mathrm{s}}$ ), and gas perturbations following the dust $\delta \mathbf{u}_{x, y} \approx-i \hat{\mu}^{1 / 2} \delta \mathbf{v}_{x, y}$ phase-shifted by $-\pi / 2$, and weaker by $\hat{\mu}^{1 / 2}$.

In Fig. 3, we show the numerically calculated dispersion relation in the very high- $\tau$ limit $(\tau=1000)$, illustrating how the cosmic-ray modes dominate over other instabilities. We also see how at low $\mu$, the resonant instability dominates (the non-resonant instability is stable for $\left.\hat{\mu}<\left(w_{\mathrm{s}} / v_{\mathrm{A}}\right)^{2}\right)$, while the non-resonant instability growth rates are much larger for sufficiently large $\mu$ and/or $w_{\mathrm{s}} / v_{\mathrm{A}}$ (not shown).

\section{THE MHD-WAVE (ALFVÉN, FAST, AND SLOW) MODES}

\subsection{Overview}

As discussed in Section 1, the basic idea of the RDI is that, although there are often several unstable modes (at any wavelength) in the coupled dust-gas system, the fastest-growing modes at a given wavelength will (usually) be those which, to leading order, simultaneously satisfy the dispersion relation of the gas, absent dust (i.e. represent 'natural' modes of the gas) and the dispersion relation of the dust, absent gas

\footnotetext{
${ }^{11}$ Note that because we have assumed the dust to have zero temperature (i.e. it is a pressure-less fluid), our treatment captures only the $n=0$ resonance from Kulsrud \& Pearce (1969).

${ }^{12}$ Again, because we assume a zero-temperature distribution function of the dust (or cosmic rays) throughout our analysis (as well as neglecting relativistic effects) our dispersion relation is slightly different from fig. 2 of Bell (2004), although it shows the same broad features.
} 
perturbations (i.e. 'natural' modes of the dust). This is especially true if the modes are both undamped, so there is no natural 'competing' damping force. A more formal discussion of these ideas is given in Appendix B.

If there were no 'back-reaction' term (force from the dust on the gas), then the gas perturbations and dust perturbations would form two entirely separable systems. The dispersion relation for gas would simply be the usual MHD relation:

$0=\left(\omega_{\mathrm{g}}^{2}-\left(v_{\mathrm{A}} \mathbf{k} \cdot \hat{\mathbf{B}}_{0}\right)^{2}\right)\left(\beta \omega_{\mathrm{g}}^{4}-\omega_{\mathrm{g}}^{2} k^{2} v_{\mathrm{A}}^{2}(1+\beta)+v_{\mathrm{A}}^{4} k^{2}\left(\mathbf{k} \cdot \hat{\mathbf{B}}_{0}\right)^{2}\right)$

which has the familiar, un-damped solutions $\omega_{\mathrm{g}}=\mathbf{v}_{\mathrm{p}} \cdot \mathbf{k}$ with $\mathbf{v}_{p}=\left( \pm \mathbf{v}_{\mathrm{A}}, \pm \mathbf{v}_{+}(\hat{\mathbf{k}}), \pm \mathbf{v}_{-}(\hat{\mathbf{k}})\right)$, i.e. the standard constant-phase velocity ideal MHD Alfvén $\left(\mathbf{v}_{\mathrm{A}} \equiv v_{\mathrm{A}} \hat{\mathbf{B}}_{0}\right)$, fast ( $\mathbf{v}_{+}$in the direction $\left.\hat{\mathbf{k}}\right)$, or slow $\left(\mathbf{v}_{-}\right)$magnetosonic waves. Meanwhile the dust, responding to a uniform (non-perturbed) gas background, would have dispersion relation

$0=\left(\omega_{\mathrm{d}}-\mathbf{k} \cdot \mathbf{w}_{\mathrm{s}}\right) \times\left[\left(\omega_{\mathrm{d}}-\mathbf{k} \cdot \mathbf{w}_{s}+i\left\langle t_{\mathrm{s}}\right\rangle^{-1}\right)^{2}\left(\omega_{\mathrm{d}}-\mathbf{k} \cdot \mathbf{w}_{\mathrm{s}}+i\left\{1+\zeta_{w}\right\}\left\langle t_{\mathrm{s}}\right\rangle^{-1}\right)-\frac{1}{\left\langle t_{\mathrm{L}}\right\rangle^{2}}\left(\omega_{\mathrm{d}}-\mathbf{k} \cdot \mathbf{w}_{\mathrm{s}}+i\left\{\cos ^{2} \theta_{\mathbf{B w}}+\zeta_{w}\right\}\left\langle t_{\mathrm{s}}\right\rangle^{-1}\right)\right]$

This has one un-damped mode, $\omega_{\mathrm{d}}=\mathbf{k} \cdot \mathbf{w}_{\mathrm{s}}$ (simple advection with the drift). It also has three damped solutions, which if we take $\hat{\mathbf{B}}_{0}=\hat{\mathbf{w}}_{\mathrm{s}}$ for simplicity can be easily solved as $\omega_{d}=\mathbf{k} \cdot \mathbf{w}_{\mathrm{s}}-i \tilde{\zeta}_{w}\left\langle t_{\mathrm{s}}\right\rangle^{-1}$ ('normal' damped motion on the stopping time) and $\omega_{\mathrm{d}}=\mathbf{k} \cdot \mathbf{w}_{\mathrm{s}} \pm\left\langle t_{\mathrm{L}}\right\rangle^{-1}-i\left\langle t_{\mathrm{s}}\right\rangle^{-1}$ (gyro motion damped on the stopping time).

The MHD-wave RDI modes are those that, 'at resonance,' simultaneously satisfy $\omega=\mathbf{k} \cdot \mathbf{w}_{\mathrm{s}}$ (the un-damped dust mode) and any of $\omega= \pm\left(\mathbf{v}_{\mathrm{A}}, \mathbf{v}_{+}, \mathbf{v}_{-}\right) \cdot \mathbf{k}$, to leading order. For the MHD-wave modes, this is possible when the mode propagates at an appropriate angle, so that:

$\mathbf{w}_{\mathrm{s}} \cdot \mathbf{k}= \pm\left(\mathbf{v}_{\mathrm{A}}, \mathbf{v}_{+}, \mathbf{v}_{-}\right) \cdot \mathbf{k}$.

Because the Alfvén and slow magnetosonic waves have phase velocities that become vanishingly small at certain angles, solutions to equation (26) always exist, for any finite $\left|\mathbf{w}_{\mathrm{s}}\right|$ and $\beta .^{13}$

Figs 2, 4, and 5, show the results of direct numerical solutions of the full dispersion relation, for the MHD-wave RDI modes. As shown explicitly from Figs 4 and 5, or by comparing the bottom-right panel of Fig. 2 to the other panels, the growth rates are almost always maximized (at a given $k$ ) at the 'resonant angles' where the condition in equation (26) is met. These numerical solutions also illustrate that at any finite $k$ and $w_{\mathrm{s}}$, there will generally be several resonant 'families.' Some range of mode angles $\hat{\mathbf{k}}$ will always satisfy the resonance condition with the slow and Alfvén waves, so these will produce a range of angles that meet the resonance condition (with different phase velocities) for both the \pm solutions of both wave families. If $w_{\mathrm{s}}$ is sufficiently large, it is also possible to meet the resonance condition with the fast wave family over some range of angles. This rich and complex resonance structure is very different from a pure hydrodynamical system (the acoustic RDI in Paper II). Because a neutral gas has only one direction-independent wavespeed $\left(c_{\mathrm{s}}\right)$, there is only one resonant family. This exists only when $w_{\mathrm{s}}>c_{\mathrm{s}}$ and features just one 'resonant angle,' $\theta_{\mathbf{a k}}=\cos ^{-1}\left(c_{\mathrm{s}} / w_{\mathrm{s}}\right)$.

\subsubsection{The mid-and short-wavelength RDI modes}

As also occurs for the acoustic RDI (see Paper I and Paper II), depending on the mode wavenumber in comparison to other scales in the problem (i.e. $c_{\mathrm{s}}\left\langle t_{\mathrm{s}}\right\rangle$, and various combinations of other parameters), there are two regimes of the MHD-wave RDIs. We term these the mid- $k$ and high- $k$ (or mid- and short-wavelength), RDIs, and explore their properties separately in Sections 5.3.1 and 5.4.1, respectively. ${ }^{14}$ They are distinguished by the scaling of the growth rate with $\mu$ (and $k$ ): in the mid- $k$ regime $\Im(\omega) \sim \mu^{1 / 2}$, while in the high- $k$ regime $\Im(\omega) \sim \mu^{1 / 3}$. As explained from the matrix-analysis perspective in Appendix B (see Appendix B3 for a simple outline), the transition between the two regimes is most simply understood by asking about the magnitude of the perturbation to the frequency (i.e. effectively the magnitude of $\Im(\omega)$ ) in comparison to other parameters in the problem, as opposed to the wavenumber itself. In particular, the mid- $k$ regime generally applies when $\Im(\omega) \lesssim\left\langle t_{\mathrm{s}}\right\rangle^{-1}$, while the high- $k$ regime applies if $\Im(\omega) \gtrsim \operatorname{MAX}\left(\left\langle t_{\mathrm{s}}\right\rangle^{-1},\left\langle t_{\mathrm{L}}\right\rangle^{-1}\right)$. If $\tau \gtrsim 1$, there is often a transition regime with $\left\langle t_{\mathrm{s}}\right\rangle^{-1} \lesssim$ $\Im(\omega) \lesssim \tau\left\langle t_{\mathrm{s}}\right\rangle^{-1}=\left\langle t_{\mathrm{L}}\right\rangle^{-1}$ where no clear scaling applies. While these conditions can be used as a general guide, we caution that they do not apply near certain special points in parameter space (e.g. when certain combinations of the $\zeta_{X}$ parameters are nearly zero; see Paper II).

\footnotetext{
${ }^{13}$ Just as in Paper II, we can also (much more tediously) derive the resonance condition directly from expansion of the 10th-order dispersion relation. To illustrate this, consider just the limit of arbitrarily high- $k$, where the dispersion relation to leading order becomes just:

$0=\left(\omega-\mathbf{k} \cdot \mathbf{w}_{\mathrm{s}}\right)^{4}\left(\omega^{2}-\left(v_{\mathrm{A}} \mathbf{k} \cdot \hat{\mathbf{B}}_{0}\right)^{2}\right)\left(\beta \omega^{4}-\omega^{2} k^{2} v_{\mathrm{A}}^{2}(1+\beta)+v_{\mathrm{A}}^{4} k^{2}\left(\mathbf{k} \cdot \hat{\mathbf{B}}_{0}\right)^{2}\right)+\mathcal{O}\left(\hat{\mu} k^{9}\right)$.

This is just the product of the (dust-free) MHD dispersion relation and $\left(\omega-\mathbf{k} \cdot \mathbf{w}_{\mathrm{s}}\right)^{4}$, so is solved either by any of the MHD modes or $\omega=\mathbf{k} \cdot \mathbf{w}_{\mathrm{s}}$. Inserting this and then expanding to next-to-leading order in $k$, we obtain the next-order correction to $\omega$. As for the acoustic RDI, these next-order terms almost always include multiple unstable modes, but for a random mode angle $\hat{\mathbf{k}}$ these have growth rates that either become independent of $k$ or are stabilized at sufficiently high $k$ (this arises from the next-to-leading term). However, if we simultaneously satisfy $\omega=\mathbf{k} \cdot \mathbf{w}_{\mathrm{s}}$ and $\omega=\omega_{\mathrm{g}}$ (the resonant condition), we eliminate multiple additional powers in equation (27) and eliminate the next-to-leading order terms (which tend to be stabilizing). Physically, we eliminate the 'natural response' of the gas that would otherwise suppress growth at high- $k$.

${ }^{14}$ Recall that the the long-wavelength, low- $k$ modes do not arise from resonances at all; see Section 4.1.
} 
This change in scaling can be clearly seen in Fig. 2. For the $\tau=0$ and $\tau=1$ cases in all three resonant families, there is a clear change in scaling at $\Im(\omega)\left\langle t_{\mathrm{s}}\right\rangle \sim 1$ from mid- $k\left(\Im(\omega) \sim k^{1 / 2}\right)$ to high- $k\left(\Im(\omega) \sim k^{1 / 3}\right)$ scaling (note that the $\tau=0$ Alfvén-wave mid- $k$ RDI has zero growth rate, so is a special case; see Section 5.3.2). For the $\tau=100$ examples, the high- $k$ scaling applies only once $\Im(\omega)\left\langle t_{\mathrm{s}}\right\rangle \gtrsim \tau=100$, which is most clearly seen on the fast-wave resonance panel (but continuing the Alfvén- and slow-wave resonance panels to higher wavenumbers shows that it does occur for these cases also).

\subsection{Resonant mode angles}

Because the gas dispersion relation for $\omega_{\mathrm{g}}$ (absent dust) has six branches, each of which has an angle-dependent phase velocity; there are in fact always a range of angles $\hat{\mathbf{k}}$ that satisfy equation (26), and therefore produce resonance. As a general rule, the angle $\hat{\mathbf{k}}$ that produces the fastest-growing mode at a given $k$ is (usually) that which produces the largest phase velocity $\left|v_{\mathrm{p}}\right|=\left|\omega_{\mathrm{g}}\right| / k=\left|\mathbf{w}_{\mathrm{s}} \cdot \hat{\mathbf{k}}\right|$, while still satisfying the resonant condition (while somewhat difficult, it is possible to read this off of Figs 4 and 5, for example). In general, this must be solved numerically, but it is instructive to consider some limits.

(i) Fast drift $\left(\left|\mathbf{w}_{\mathrm{s}}\right| \gtrsim v_{\mathrm{f}, 0}\right)$ : In this case the fastest resonance is with the fast magnetosonic wave. That wave has a phase speed which is only weakly sensitive to angle (between $v_{\mathrm{f}, 0}=\sqrt{c_{\mathrm{s}}^{2}+v_{\mathrm{A}}^{2}}$ and $\mathrm{MAX}\left(v_{\mathrm{A}}, c_{\mathrm{s}}\right)$ ). So the resonant angle must obey, approximately:

$$
\begin{aligned}
& \cos \theta_{\mathbf{w k}}=\hat{\mathbf{k}} \cdot \hat{\mathbf{w}}_{\mathrm{s}}=\frac{k_{z}}{k} \approx \pm \frac{v_{\mathrm{f}, 0}}{w_{\mathrm{s}}}, \\
& \hat{\mathbf{k}} \cdot \mathbf{w}_{\mathrm{s}}= \pm v_{+}(\hat{\mathbf{k}}) \approx \pm v_{\mathrm{f}, 0} .
\end{aligned}
$$

The \pm directions behave identically here. The weak sensitivity of $v_{+}$to angle means that the orientation of the component of $\hat{\mathbf{k}}$ perpendicular to $\hat{\mathbf{w}}_{\mathrm{S}}$ (the angle of $\mathbf{k}_{\perp}$ in the $x-y$ plane) usually has only a small effect on the growth rates. The phase speed of $v_{+}$is maximized when the projection onto $\hat{\mathbf{B}}_{0}$ is minimized, so the growth rates are usually slightly higher for modes with the perpendicular component $\mathbf{k}_{\perp}$ oriented primarily along the direction mutually perpendicular to $\hat{\mathbf{w}}_{\mathrm{s}}$ and $\mathbf{B}_{0}$ (i.e. the $\hat{y} \propto \mathbf{w}_{\mathrm{s}} \times \mathbf{B}_{0}$ direction), while the projection onto $k_{x}$ satisfies $k_{x} / k \approx-\left(\cot \theta_{\mathbf{B w}}\right)\left(k_{z} / k\right)$ (so $\left.\hat{\mathbf{k}} \cdot \hat{\mathbf{B}}_{0} \approx 0\right)$.

(ii) Intermediate drift, strongly magnetized $\left(c_{\mathrm{s}} \ll\left|\mathbf{w}_{\mathrm{s}}\right| \ll v_{\mathrm{A}}\right)$ : In this case (which can occur at low- $\beta$ ), the drift is faster than the sound speed but slower than Alfvén, prohibiting resonance with the fast magnetosonic wave. The slow magnetosonic wave speed is maximized at $\operatorname{MIN}\left(c_{\mathrm{s}}, v_{\mathrm{A}}\right)=c_{\mathrm{s}}$, while the Alfvén wave has phase velocity $=v_{\mathrm{A}} \cos \theta_{\mathbf{B k}}$ (which can be much larger than the slow wave). Thus, the fastest resonance is with the Alfvén wave, leading to the resonance requirement $k_{x}=k_{z}\left(w_{\mathrm{s}} / v_{\mathrm{A}}-\cos \theta_{\mathbf{B w}}\right) / \sin \theta_{\mathbf{B w}}$. Now, recall we wish to maximize $\left|\hat{\mathbf{k}} \cdot \mathbf{w}_{\mathrm{s}}\right| \propto\left|k_{z}\right|$, but we must obey $k_{z}^{2}+k_{x}^{2} \leq k^{2}$; maximizing $\left|k_{z}\right|$ subject to this constraint gives $\hat{\mathbf{k}}=\left(k_{x}, k_{y}, k_{z}\right)$ with $k_{y}=0$ and $k_{z}= \pm\left[1+\left(w_{\mathrm{s}} / v_{\mathrm{A}}-\cos \theta_{\mathbf{B w}}\right)^{2} / \sin ^{2} \theta_{\mathbf{B W}}\right]^{-1 / 2}$, or (to leading order):

$$
\hat{\mathbf{k}} \approx \pm\left(-\cos \theta_{\mathbf{B w}}+\frac{w_{\mathrm{s}}}{v_{\mathrm{A}}} \sin ^{2} \theta_{\mathbf{B w}}, 0, \sin \theta_{\mathbf{B w}}\left[1+\frac{w_{\mathrm{s}}}{v_{\mathrm{A}}} \cos \theta_{\mathbf{B w}}\right]\right),
$$

$\hat{\mathbf{k}} \cdot \mathbf{w}_{\mathrm{s}}=\mathbf{v}_{\mathrm{A}}(\hat{\mathbf{k}}) \approx \pm w_{\mathrm{s}} \sin \theta_{\mathbf{B w}}$

so $\left|\cos \theta_{\mathbf{B k}}\right| \approx\left(w_{\mathrm{s}} / v_{\mathrm{A}}\right)\left|\sin \theta_{\mathbf{B w}}\right| \ll 1$. Note that our sign convention is such that $\sin \theta_{\mathbf{B w}}>0$ always (i.e. $\left.\sin \theta_{\mathbf{B w}} \equiv \sqrt{1-\cos \theta_{\mathbf{B w}}^{2}}\right)$.

In short, the fastest-growing mode is oriented almost (but not quite) perpendicular to $\hat{\mathbf{B}}_{0}$ in the $\hat{\mathbf{B}}_{0}-\hat{\mathbf{w}}_{s}$ plane.

(iii) Intermediate drift, weakly-magnetized $\left(v_{\mathrm{A}} \ll\left|\mathbf{w}_{\mathrm{s}}\right| \ll c_{\mathrm{s}}\right)$ : In this case, resonance with the fast wave is not possible, but resonances with either the Alfvén or slow modes are possible, and these modes have nearly identical phase speeds (since $\beta \gg 1$ ). Thus the Alfvén and slow resonances are essentially degenerate. We again have $\mathbf{w}_{\mathrm{s}} \cdot \mathbf{k} \approx v_{\mathrm{A}} \cos \theta_{\mathbf{B k}}$, or $k_{x}=k_{z}\left(w_{\mathrm{s}} / v_{\mathrm{A}}-\cos \theta_{\mathbf{B w}}\right) / \sin \theta_{\mathbf{B w}} ;$ maximizing $\left|k_{z}\right|$ again gives $k_{y}=0, k_{z}= \pm\left[1+\left(w_{\mathrm{s}} / v_{\mathrm{A}}-\cos \theta_{\mathbf{B w}}\right)^{2} / \sin ^{2} \theta_{\mathbf{B W}}\right]^{-1 / 2}$. Then, because $w_{\mathrm{s}} \gg v_{\mathrm{A}}$, to leading order the maximum phase speed occurs at

$$
\hat{\mathbf{k}} \approx \pm\left(1-\frac{v_{\mathrm{A}}^{2} \sin ^{2} \theta_{\mathbf{B w}}}{2 w_{\mathrm{s}}^{2}}, 0, \frac{v_{\mathrm{A}} \sin \theta_{\mathbf{B w}}}{w_{\mathrm{s}}}\right),
$$

$\hat{\mathbf{k}} \cdot \mathbf{w}_{\mathrm{s}}=v_{-}(\hat{\mathbf{k}}) \approx \pm v_{\mathrm{A}} \sin \theta_{\mathbf{B w}}$.

So, the fastest-growing mode is has $\hat{\mathbf{k}}$ primarily in the direction of $\mathbf{B}_{\perp}$ (the direction of $\mathbf{B}_{0}$ perpendicular to $\mathbf{w}_{\mathrm{s}}$ ).

(iv) Slow drift $\left(\left|\mathbf{w}_{\mathrm{s}}\right| \ll \operatorname{MIN}\left(c_{\mathrm{s}}, v_{\mathrm{A}}\right)\right)$ : For small $\left|\mathbf{w}_{\mathrm{s}}\right| \lesssim \operatorname{MIN}\left(c_{\mathrm{s}}, v_{\mathrm{A}}\right)$, resonance with the fast magnetosonic wave is not possible and resonance with the slow or Alfvén waves requires $\left|\cos \theta_{\mathbf{B k}}\right| \ll 1$ (so that the phase speed is low). Thus, the slow-wave phase speed is again given by an expression similar to the Alfvén-wave phase speed, $v_{-}^{2} \approx\left(c_{\mathrm{s}} v_{\mathrm{A}} / v_{\mathrm{f}, 0}\right)^{2} \cos ^{2} \theta_{\mathbf{B k}}$. Setting this equal to $\left(\hat{\mathbf{k}} \cdot \mathbf{w}_{\mathrm{s}}\right)^{2}$ we obtain the requirement $\left(k_{x} / k\right)=\left(k_{z} / k\right)\left(-\cos \theta_{\mathbf{B w}} \pm \tilde{w}\right) /\left|\sin \theta_{\mathbf{B w}}\right|\left(\right.$ where $\left.\tilde{w} \equiv\left|\mathbf{w}_{\mathrm{s}}\right| v_{\mathrm{f}, 0} /\left(c_{\mathrm{s}} v_{\mathrm{A}}\right) \approx\left|\mathbf{w}_{\mathrm{s}}\right| / \operatorname{MIN}\left(c_{\mathrm{s}}, v_{\mathrm{A}}\right)\right)$. We then obtain

$$
\hat{\mathbf{k}} \approx \pm\left(-\cos \theta_{\mathbf{B w}}+\tilde{w} \sin ^{2} \theta_{\mathbf{B w}}, 0, \sin \theta_{\mathbf{B w}}\left(1+\tilde{w} \cos \theta_{\mathbf{B w}}\right)\right)
$$

$\hat{\mathbf{k}} \cdot \mathbf{w}_{\mathrm{s}}= \pm v_{-}(\hat{\mathbf{k}}) \approx \pm w_{\mathrm{s}} \sin \theta_{\mathbf{B w}}$.

Like the intermediate-drift, strongly magnetized case, the fastest-growing mode is oriented close to perpendicular to $\hat{\mathbf{B}}_{0}$ in the $\hat{\mathbf{B}}_{0}-\hat{\mathbf{w}}_{s}$ plane. Note that both the Alfvén and slow mode resonances have a similar resonant angle in this case, but at low $\beta$ the growth rates can be different. 


\subsection{Growth rates: the mid-wavelength (low- $\mu)$ MHD-wave RDI modes}

With Section 5.2 in mind, if we expand the dispersion relation about $\hat{\mu} \ll 1$, and assume the resonance condition - i.e. $\mathbf{w}_{\mathrm{s}} \cdot \hat{\mathbf{k}}=v_{ \pm}$or $v_{\mathrm{A}}$ (matching the fast, slow, or Alfvén phase velocity) - then we obtain a leading-order dispersion relation of the form:

$\omega_{ \pm, \mathrm{A}}=k v_{ \pm, \mathrm{A}}+(i \pm 1) \mathcal{F}_{\text {mid }}\left(\frac{\hat{\mu} k v_{f, 0}}{2\left\langle t_{\mathrm{s}}\right\rangle}\right)^{1 / 2}+\mathcal{O}(\hat{\mu})$.

This always has an unstable root, as with the long-wavelength mode. ${ }^{15}$ As explained in detail in Appendix B (see also Section 5.1.1), the expansion used to derive equation (32) is generally valid when the derived perturbation to $\omega_{ \pm, \mathrm{A}}$ (i.e. $\omega_{ \pm, \mathrm{A}}-k v_{ \pm, \mathrm{A}}$ ) is less than $\sim\left\langle t_{\mathrm{s}}\right\rangle^{-1}$, but is still less than the long-wavelength, low- $k$ growth-rate prediction (see Section 4.1 and equation 16). We will now consider the cases where the resonance is with the (fast or slow) magnetosonic, or Alfvén phase speeds.

\subsubsection{The (fast and slow) magnetosonic-wave RDI}

First consider the case of modes resonant with the magnetosonic phase velocities: $\mathbf{w}_{\mathrm{s}} \cdot \hat{\mathbf{k}}=v_{ \pm}$, where we will consider the most relevant cases of the fast-mode resonance when $w_{\mathrm{s}} \gtrsim v_{\mathrm{f}, 0}$ (since this is the fastest-growing resonance) and slow-mode resonance when $w_{\mathrm{s}} \lesssim v_{\mathrm{f}, 0}$. Even restricting to the magnetosonic RDI in the mid-wavelength regime, the expressions for $\mathcal{F}_{\text {mid }}$ are rather un-informative, so we will further consider the limits of weak and strong Lorentz forces.

(i) Weak Lorentz forces $(\tau \ll 1)$ : If we neglect Lorentz forces, then the growth rates for this mode simplify to the general expression from Paper I:

$$
\begin{aligned}
\mathcal{F}_{\text {mid }} & =\left|\left(1-\zeta_{\mathrm{s} / \mathrm{w}}\right)\left(\frac{v_{\mp}^{2} \cos \theta_{\mathbf{B a}}}{\cos \theta_{\mathbf{B k}} \cos \theta_{\mathbf{k a}}}-1\right) \Theta_{ \pm}\right|^{1 / 2} \\
\Theta_{ \pm} & \equiv \frac{v_{ \pm^{3}}\left(1-v_{\mp}^{2}\right)}{\left(1+v_{\mp}^{2}\right)^{2}+v_{ \pm}^{2}\left(1-2 v_{\mp}^{2}\right)+\sin ^{2} \theta_{\mathbf{B k}} / \beta+v_{\mp}^{4} v_{ \pm}^{2} / \cos ^{2} \theta_{\mathbf{B k}}}
\end{aligned}
$$

(this expression is valid for any angle that satisfies the resonant condition). But even this is rather un-intuitive. To simplify further, consider the fastest-growing resonant angle in both the 'fast drift' (resonance with the fast magnetosonic mode) and 'slow drift' (slow mode resonance) limits (Section 5.2). Equation (33) then becomes

$\mathcal{F}_{\text {mid }} \approx \begin{cases}\left|1-\zeta_{\mathrm{s} / \mathrm{w}}\right|^{1 / 2} & \left(w_{\mathrm{s}} \gg v_{\mathrm{f}, 0}\right) \\ \frac{w_{0}}{v_{\mathrm{f}, 0}}\left[\frac{\left|\left(1-\zeta_{\mathrm{s} / \mathrm{w}}\right) \sin \theta_{\mathbf{B a}} \cos \theta_{\mathbf{B a}}\right|^{1 / 2}}{\beta^{1 / 4}}\right] & \left(w_{\mathrm{s}} \ll v_{\mathrm{f}, 0}\right) .\end{cases}$

Note that for the slow-drift case, if $\sin \theta_{\mathbf{B a}}=0$ exactly (drift and field are perfectly parallel), it becomes impossible to satisfy the slow-mode resonant condition for $w_{\mathrm{s}} \lesssim \operatorname{MIN}\left(c_{\mathrm{s}}, v_{\mathrm{A}}\right)$, so the growth rate vanishes. However for $\cos \theta_{\mathbf{B a}}=0$ (exactly perpendicular drift and field lines) the resonance does not vanish (our series expansion simply becomes inaccurate), and a more accurate derivation in the limit where $\cos \theta_{\mathbf{B a}}$ is small leads to the replacement $\left|\cos \theta_{\mathbf{B a}}\right| \rightarrow \operatorname{MAX}\left[\left|\cos \theta_{\mathbf{B a}}\right|,\left(w_{\mathrm{s}} / v_{\mathrm{f}, 0}\right)\right]{ }^{16}$

(ii) Strong Lorentz Forces $(\tau \gg 1)$ : In the limit where Lorentz forces dominate $\operatorname{drag}(\tau \gg 1)$, we find

$\mathcal{F}_{\text {mid }} \approx \begin{cases}\left|\frac{\zeta_{\mathrm{s}}}{\tilde{\zeta}_{w}}\left(\frac{\beta}{1+\beta} \pm \frac{w_{0}}{v_{\mathrm{f}, 0}} \sin \theta_{\mathbf{B a}}\right)+\frac{\tan ^{2} \theta_{\mathbf{B a}}}{\tilde{\zeta}_{w}}\right|^{1 / 2} & \left(w_{\mathrm{s}} \gg v_{\mathrm{f}, 0}\right) \\ \frac{w_{0}}{v_{\mathrm{f}, 0}}\left[\frac{\left|\left(\beta+\left[1-\zeta_{q}\right]\right) \sin \theta_{\mathbf{B a}} \cos \theta_{\mathbf{B a}}\right|^{1 / 2}}{\tau^{1 / 2} \beta^{1 / 4}}\right] & \left(w_{\mathrm{s}} \ll v_{\mathrm{f}, 0}\right) .\end{cases}$

Note that if $\cos \theta_{\mathbf{B a}} \rightarrow 0, \tan ^{2} \theta_{\mathbf{B a}} \rightarrow \infty$, but the growth rates do not actually diverge (our series expansion is simply inaccurate). A more accurate expansion gives the upper and lower limits of this term of $\tan ^{2} \theta_{\mathbf{B a}} \rightarrow \operatorname{MIN}\left\{\tan ^{2} \theta_{\mathbf{B a}},\left(w_{0} / v_{\mathrm{f}, 0}\right)^{2} /(2+\beta)\right\}\left(\right.$ as $\left.\theta_{\mathbf{B a}} \rightarrow \pm \pi / 2\right)$ and $\tan ^{2} \theta_{\mathbf{B a}} \rightarrow \operatorname{MAX}\left\{\tan ^{2} \theta_{\mathbf{B a}}, \tilde{\zeta}_{w}\left(c_{\mathrm{s}} / w_{0}\right)^{2}\right\}\left(\operatorname{as} \theta_{\mathbf{B a}} \rightarrow 0, \pi\right)$

\footnotetext{
${ }^{15}$ In equation (32), note that the second-from leading term (in $i \pm 1$ ) comes from solving an equation of the form $\omega_{ \pm, \mathrm{A}}=k v_{ \pm, \mathrm{A}}+\Omega$ with $\Omega^{2}= \pm i X$, where $X=\mathcal{F}_{\text {mid }}^{2} \hat{\mu} k v_{f, 0} /\left\langle t_{s}\right\rangle$ is purely real. Unless $\mathcal{F}_{\text {mid }}=0$, this always has an unstable solution with roots proportional to $(i \pm 1) / \sqrt{2}$ where the \pm for the real part corresponds to the \pm sign of $\Omega^{2}= \pm i X$ but has no effect on the growth rate.

${ }^{16}$ Note, if $\beta$ is sufficiently large so $v_{\mathrm{A}} \ll w_{\mathrm{s}} \ll c_{\mathrm{s}}$ (so we are not cleanly in the 'slow' or 'fast' regime, the scaling is modified to $\mathcal{F}_{\text {mid }} \approx(1 / 2)(1-$ $\left.\cos ^{2} \theta_{\mathbf{B a}}\right)^{1 / 2}\left(1-\cos ^{2} \theta_{\mathbf{B a}}\right)^{1 / 4} \beta^{-3 / 4}$.
} 


\subsubsection{The Alfvén-wave RDI}

If instead the resonance is with the Alfvén phase speed $\left(\mathbf{w}_{\mathrm{s}} \cdot \mathbf{k}= \pm v_{\mathrm{A}}\right)$, the character of the modes is significantly different in some regimes. Also, the mid- $k$ Alfvén RDI vanishes entirely if $\tau=0$ because it depends on the presence of Lorentz forces on the dust. As before, the general expression is rather unintuitive so we give only the limits of weak and strong Lorentz forces.

(i) Weak Lorentz forces $\left(\tau \ll 1\right.$ ): Here, the fastest-growing modes have $\hat{k}_{y} \rightarrow \pm 1$ (with a non-zero but very small projection onto the $\hat{\mathbf{B}}_{0}-\mathbf{w}_{\mathrm{s}}$ plane), giving

$\mathcal{F}_{\text {mid }}=\frac{w_{0}}{v_{\mathrm{f}, 0}} \frac{(1+\beta)^{1 / 4}\left|\tau \sin \theta_{\mathbf{B a}} \cos \theta_{\mathbf{B a}}\right|^{1 / 2}}{\sqrt{2}}$

We see this vanishes as $\tau \rightarrow 0$, unlike the magnetosonic RDI, so at very low $\tau$ this is never the fastest-growing mode. However, also note that this expression applies for all $w_{0}$ (not just high or low $w_{0}$ ). Comparing to the magnetosonic modes (equation 34), in the 'fast' limit (where $\left.w_{0} \gg v_{\mathrm{f}, 0}\right)$, this differs from the fast-RDI by a factor $\sim\left(w_{0} / v_{\mathrm{f}, 0}\right)(1+\beta)^{1 / 4} \tau^{1 / 2}$, so if $\tau$ is not too small, the Alfvén-wave RDI can be the fastest-growing mode in the system for sufficiently large $w_{\mathrm{s}}$ or $\beta$. In the 'slow' limit $\left(w_{0} \ll v_{\mathrm{f}, 0}\right)$ the growth rate scales similarly to the slow RDI, but with an additional factor $\sim \tau^{1 / 2} \beta^{1 / 4}(1+\beta)^{1 / 4}$ - so for sufficiently large $\beta \gg 1 / \tau$ this can again be the fastest-growing mode.

(ii) Strong Lorentz forces $\left(\tau \gg 1\right.$ ): In this regime, the fastest-growing modes have $\hat{k}_{y}=0$ (oriented in the $\hat{\mathbf{B}}_{0}-\mathbf{w}_{\mathrm{s}}$ plane), giving:

$\mathcal{F}_{\text {mid }}=\frac{w_{0}}{v_{\mathrm{f}, 0}} \frac{(1+\beta)^{1 / 4}\left|\sin \theta_{\mathbf{B a}} \cos \theta_{\mathbf{B a}}\right|^{1 / 2}}{\tau^{1 / 2} \sqrt{2}}\left|s^{ \pm}+\frac{w_{\mathrm{s}} \tan ^{2} \theta_{\mathbf{B a}}}{\tilde{\zeta}_{w}\left|w_{\mathrm{s}}-v_{\mathrm{A}}\right|}\right|^{1 / 2}$,

where $s^{ \pm}=1$ if $w_{\mathrm{s}}>v_{\mathrm{A}}$ and $s^{ \pm}=-1$ if $w_{\mathrm{s}}<v_{\mathrm{A}}$. Note that this is suppressed by a power $\tau^{1 / 2}$. In the 'fast' limit that suppression means this is usually slower-growing than the fast RDI (equation 35) if $\beta$ is also large; the growth rate of the Alfvén RDI in this limit then differs by a factor $\sim\left(w_{\mathrm{s}} / \tau v_{\mathrm{A}}\right)^{1 / 2}$ so for sufficiently large $w_{\mathrm{s}} \gg \tau v_{\mathrm{A}}$ could still be fastest-growing (but this is usually not the case). In the 'intermediate' (strongly or weakly magnetized) or 'slow' limits, this is very similar to the slow-RDI.

\subsection{Growth rates: the short-wavelength (high- $k$ ) MHD-wave RDI modes}

At sufficiently short wavelengths (high $k$ ), we can expand the dispersion relation in powers of $k^{-1} \ll 1$. If we do this, and once again assume the resonance condition $\mathbf{w}_{\mathrm{s}} \cdot \hat{\mathbf{k}}=v_{ \pm}$or $v_{\mathrm{A}}$, we obtain the leading-order dispersion relation $\omega_{ \pm, \mathrm{A}}=k v_{ \pm, \mathrm{A}}+\Omega_{ \pm, \mathrm{A}}+\mathcal{O}\left(k^{0}\right)$, where $\Omega_{ \pm, \mathrm{A}}^{3}=\mathcal{Q}_{ \pm, \mathrm{A}} \mu k v_{\mathrm{f}, 0} /\left(2\left\langle t_{\mathrm{s}}\right\rangle\right)$ so $\Omega \sim \mathcal{O}\left(k^{1 / 3}\right)$. Here $\mathcal{Q}$ is a real number, so this always has unstable $(\Im(\omega)>0)$ solutions unless $\|\mathcal{Q}\|=0$ exactly. We can therefore write

$\omega_{ \pm, \mathrm{A}}=k v_{ \pm, \mathrm{A}}+\left(\frac{i \sqrt{3} \pm 1}{2}\right) \mathcal{F}_{\mathrm{hi}}\left|\frac{\mu k v_{\mathrm{f}, 0}}{2\left\langle t_{\mathrm{s}}\right\rangle^{2}}\right|^{1 / 3}+\mathcal{O}\left(k^{0}\right)$,

where $\mathcal{F}_{\mathrm{hi}}=\|\mathcal{Q}\|^{1 / 3}$ and the sign of the \pm is opposite the sign of $\mathcal{Q}$. As discussed in Section 5.1.1 and in detail in Appendix B, equation (38) is generally valid for sufficiently high $k$ such that the perturbation to the growth rate (i.e. $\left.\omega_{ \pm, \mathrm{A}}-k v_{ \pm, \mathrm{A}}\right)$ is larger than $\sim \mathrm{MAX}\left(\left\langle t_{\mathrm{s}}\right\rangle^{-1},\left\langle t_{\mathrm{L}}\right\rangle^{-1}\right)=$ $\operatorname{MAX}(1, \tau)\left\langle t_{\mathrm{s}}\right\rangle^{-1}$. As before, we consider $\mathcal{F}_{\text {hi }}$ for the fast and slow magnetosonic, or the Alfvén RDIs.

\subsubsection{The (fast \& slow) magnetosonic-wave RDI}

As before (Section 5.3.1), we first consider the magnetosonic RDI. Even with this specification, the full expression for $\mathcal{Q}$ is again rather opaque, ${ }^{17}$ so we will consider separately the limits of weak and strong Lorentz forces.

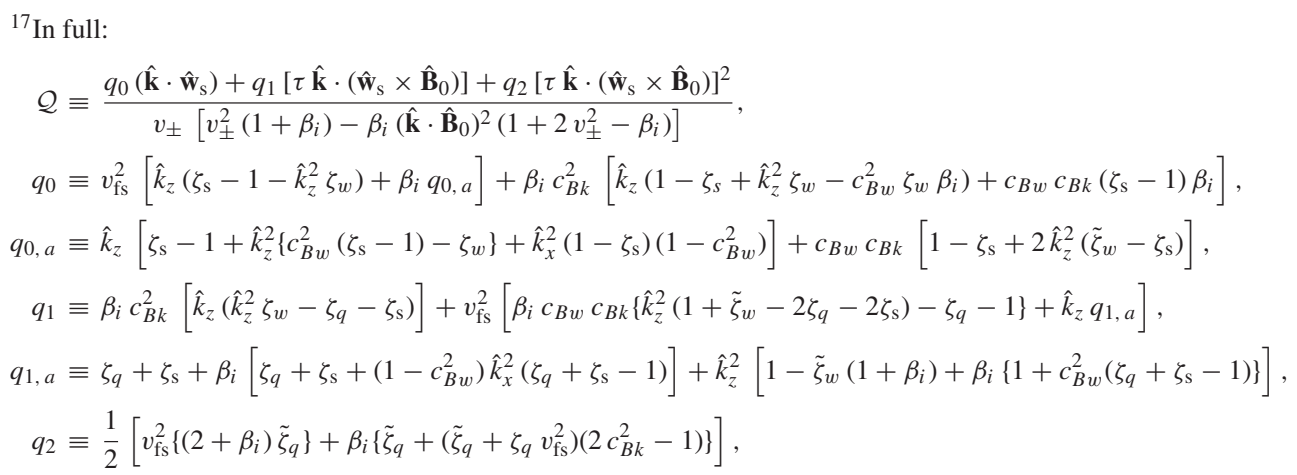

where for brevity we denoted $c_{\mathrm{Bk}}=\cos \theta_{\mathbf{B k}}, c_{\mathrm{Bw}}=\cos \theta_{\mathbf{B w}}$. 
(i) Weak Lorentz forces $(\tau \ll 1)$ : In this case ${ }^{18}$ we obtain

$$
\mathcal{F}_{\mathrm{hi}} \approx \begin{cases}\left|\frac{\left(1-\zeta_{\mathrm{s}}\right)\left(\beta \pm \sin ^{2} \theta_{\mathbf{B a}}\right)}{\beta+1}\right|^{1 / 3} & \left(w_{\mathrm{s}} \gg v_{\mathrm{f}, 0}\right) \\ \left(\frac{w_{0}}{v_{\mathrm{f}, 0}}\right)^{2 / 3} \frac{\left|\left(1-\zeta_{\mathrm{s}}\right) \sin \theta_{\mathbf{B a}} \cos \theta_{\mathbf{B a}}\right|^{1 / 3}}{\beta^{1 / 6}} & \left(w_{\mathrm{s}} \ll v_{\mathrm{f}, 0}\right)\end{cases}
$$

(ii) Strong Lorentz forces $(\tau \gg 1)$ : And in this case we obtain

$$
\mathcal{F}_{\mathrm{hi}} \approx \begin{cases}\left(\frac{w_{0} \sin \theta_{\mathbf{B a}}}{v_{\mathrm{f}, 0}}\right)^{2 / 3}\left(1+\zeta_{q}\right)^{1 / 3} & \left(w_{\mathrm{s}} \gg v_{\mathrm{f}, 0}\right) \\ \left(\frac{w_{0}}{v_{\mathrm{f}, 0}}\right)^{2 / 3} \frac{\left|\left(\zeta_{q}-\beta\right) \sin \theta_{\mathbf{B a}} \cos \theta_{\mathbf{B a}}\right|^{1 / 3}}{\beta^{1 / 6}} & \left(w_{\mathrm{s}} \ll v_{\mathrm{f}, 0}\right)\end{cases}
$$

\subsubsection{The Alfvén-wave RDI}

As with the mid- $k$ mode, the Alfvén RDI exhibits significantly different character (compared to the magnetosonic RDI) in the high- $k$ regime. The general expression is once again not particularly informative, although we note that for $\hat{k}_{y}=0$ (not necessarily the fastest-growing case), it simplifies dramatically to $\mathcal{Q}=\hat{k}_{z}\left(w_{\mathrm{s}} / v_{\mathrm{f}, 0}\right) \tau^{2}\left[1-2\left(w_{\mathrm{s}} / v_{\mathrm{A}}\right) \cos \theta_{\mathbf{B w}}+\left(w_{\mathrm{s}} / v_{\mathrm{A}}\right)^{2}\right]$. It is also worth noting that the high- $k$ Alfvén RDI does not vanish (if $\hat{k}_{y} \neq 0$ ) in the limit of uncharged grains $(\tau=0)$, unlike its mid- $k$ cousin.

(i) Weak Lorentz forces $(\tau \ll 1)$ : Here the growth rate vanishes if any single component of $\hat{\mathbf{k}}$ does; the maximum growth rate occurs when $\hat{k}_{y}^{2} \approx 1 / 3 \rightarrow 2 / 3$ (depending on $\beta$ ). To simplify the expression, take $\hat{k}_{y}^{2}=2 / 3$ (the effect of changing to $\hat{k}_{y}^{2}=1 / 3$ is less than a factor of 2 here for all $\beta$ ). Then the growth rate becomes

$\mathcal{F}_{\mathrm{hi}} \approx \frac{\left(\sin \theta_{\mathbf{B a}}\right)^{1 / 2}\left|\zeta_{w}\right|^{1 / 3}}{(\sqrt{3} / 2)} \min \left\{\frac{w_{\mathrm{s}}}{v_{\mathrm{f}, 0}}, \frac{(1+\beta)^{-1 / 2}}{1-\left(\sin ^{2} \theta_{\mathbf{B a}}\right) / 3}\right\}^{1 / 3}$

As we noted above, we see that this is independent of $\tau$, so the instability (unlike the mid- $k$ Alfvén wave RDI) does not vanish in the limit of uncharged grains $(\tau=0)$. Its growth does, however, rely on the velocity dependence of the dust drag law, since it is proportional to $\zeta_{w}=0$. Also note that, unlike the magnetosonic RDI (where the fast and slow RDI had different scalings), the expression above applies for all values of $w_{0}$. So for the 'fast' limit $\left(w_{\mathrm{s}} \gg v_{\mathrm{f}, 0}\right), \mathcal{F}_{\mathrm{hi}}$ scales very similarly to the fast magnetosonic RDI with low- $\tau$ (equation 39$)$. Although this differs from equation (41) in this limit only by order-unity constants and the scaling coefficients $\zeta$, as noted in Section 9.1.5 below, if Epstein drag dominates (as it usually does at high- $k$ ) then $1-\zeta_{\mathrm{s}}$ (which appears in equation (39)) scales $\propto\left(c_{\mathrm{s}} / w_{\mathrm{s}}\right)^{2}$ for $w_{\mathrm{s}} \gg c_{\mathrm{s}}$. So the fast RDI has a somewhat suppressed growth rate in this limit, while the Alfvén RDI (whose pre-factor $\zeta_{w} \approx 1$ for Epstein drag with $w_{\mathrm{s}} \gg c_{\mathrm{s}}$ ) is not suppressed by any power of $w_{\mathrm{s}}$.

In the intermediate, strongly magnetized limit $\left(c_{\mathrm{s}} \ll w_{\mathrm{s}} \ll v_{\mathrm{A}}\right)$, where the fast RDI is not possible, however, equation (41) scales as $\sim\left(w_{\mathrm{s}} / v_{\mathrm{f}, 0}\right)^{1 / 3}$, so can lead to a larger growth rate than the slow-RDI (equation 39) by a factor $\sim\left(v_{\mathrm{f}, 0} / w_{\mathrm{s}}\right)^{1 / 3} \gg 1$. In other words, the growth rate is less strongly suppressed for the Alfvén RDI than for the slow RDI. This is because (as discussed in Section 5.2), in this particular limit the resonance with the Alfvén wave has much higher phase velocity than the resonance with the slow wave because $v_{\mathrm{A}} \gg c_{\mathrm{s}}$.

For the intermediate, weakly magnetized $\left(v_{\mathrm{A}} \ll w_{\mathrm{s}} \ll c_{\mathrm{S}}\right)$ or 'slow' limits $\left(w_{\mathrm{s}} \ll \min \left\{c_{\mathrm{s}}, v_{\mathrm{A}}\right\}\right)$, we have $\zeta_{w} \propto\left(w_{\mathrm{s}} / c_{\mathrm{S}}\right)^{2} \rightarrow\left(w_{\mathrm{s}} / c_{\mathrm{s}}\right)^{3} \ll 1($ with the exponent $=2$ for Epstein/Stokes drag or $=3$ for Coulomb drag). Thus $\mathcal{F}_{\text {hi }} \propto\left(w_{\mathrm{s}} / c_{\mathrm{s}}\right)^{\alpha}$ with $\alpha=1$ or $4 / 3$, and the growth rates of the Alfvén RDI are suppressed relative to the slow RDI.

(ii) Strong Lorentz forces $(\tau \gg 1)$ : In this limit, we find

$\mathcal{F}_{\text {hi }}=\left|\frac{w_{0}}{v_{\mathrm{f}, 0}} \tau \sin \theta_{\mathbf{B a}}\left(1-\frac{w_{0} \cos \theta_{\mathbf{B a}}}{v_{\mathrm{A}}}\right)\right|^{1 / 3}$.

Again, this expression applies for all $w_{0}$. This means that in the 'fast' case $\left(w_{0} \gg v_{\mathrm{A}}\right.$ or $\left.v_{\mathrm{f}, 0}\right)$, the Alfvén RDI has a growth rate that scales $\propto\left[\tau w_{0}^{2} /\left(v_{\mathrm{f}, 0} v_{\mathrm{A}}\right)\right]^{1 / 3}$ - dimensionally, this is larger than the fast-magnetosonic RDI (equation 40 ) by a factor $\sim \tau^{1 / 3}(1+\beta)^{1 / 6}$. For 'intermediate' cases with $v_{\mathrm{A}} \ll w_{\mathrm{s}} \ll c_{\mathrm{s}} \sim v_{\mathrm{f}, 0}$, the fast-magnetosonic RDI is not possible and the Alfvén RDI has a growth rate faster than the slow-magnetosonic RDI by a factor $\sim \tau^{1 / 3}$; for 'slow' cases with $w_{\mathrm{s}} \ll \min \left\{c_{\mathrm{s}}, v_{\mathrm{A}}\right\}$, the Alfvén RDI has a growth rate larger than the slow-RDI by a factor $\sim \tau^{1 / 3}\left(v_{\mathrm{f}, 0} / w_{0}\right)^{1 / 3}$. So at sufficiently high- $\tau$, this is usually the fastest-growing mode. Effectively, in these cases, $\left\langle t_{\mathrm{s}}\right\rangle^{2}$ in the denominator of the growth rate (equation 38) is replaced by $\left\langle t_{\mathrm{s}}\right\rangle\left\langle t_{\mathrm{L}}\right\rangle$ (the geometric mean). This implies that mode growth timescales can

\footnotetext{
${ }^{18}$ The scaling shown for the slow-drift limit in equation (39) assumes $\zeta_{w} \approx 0$, which is applicable for sub-sonic Epstein or Stokes or Coulomb drag. Since the slow-mode resonance limit is (by definition) sub-sonic, we have expanded assuming that one of these laws is true. But if the scaling of the drag law were such that $\zeta_{w}$ were significantly non-zero at order larger than $w_{\mathrm{s}} / v_{\mathrm{f}, 0}$, then there is a less strongly suppressed term and the leading-order term for the slow limit in equation (39) is only suppressed as $\sim\left(\zeta_{w} w_{\mathrm{s}} / v_{\mathrm{f}, 0}\right)^{1 / 3}$, as opposed to $\sim\left(w_{\mathrm{s}} / v_{\mathrm{f}, 0}\right)^{2 / 3}$.
} 
become comparable to the gyro timescale, rather than the (slower) stopping time. The limitation is that this large enhancement in the growth rate only occurs at very short wavelengths, $k \gtrsim \tau^{3} /\left(v_{\mathrm{f}, 0}\left\langle t_{\mathrm{s}}\right\rangle\right)$.

\subsection{Mode structure}

In this section, we briefly discuss the structure of the MHD-wave RDI modes.

(i) Fast-wave RDI: Around resonance, the phase velocity is approximately that of a fast-magnetosonic wave $\mathbf{v}_{\mathrm{p}} \approx \mathbf{v}_{+}$. Like we saw for the acoustic RDI in Paper II, the gas perturbation $(\delta \rho, \delta \mathbf{B}, \delta \mathbf{u})$ increasingly resembles a simple, pure fast-magnetosonic wave for larger $k$. Also like the acoustic RDI, the perturbed dust velocity $\delta \mathbf{v}$ is smaller (by $\sim \hat{\mu}^{1 / 2}$ ) and out-of-phase with $\delta \mathbf{u}\left(\right.$ leading by $\sim 150^{\circ}$ ) while the dust density $\delta \rho_{\mathrm{d}}$ leads $\delta \rho$ by $\sim 30^{\circ}$ with a very large amplitude: $\left|\delta \rho_{\mathrm{d}}\right| /|\mu \delta \rho| \sim \Im\left(\omega\left\langle t_{\mathrm{s}}\right\rangle\right) / \mu$, which translates to $\left|\delta \rho_{\mathrm{d}}\right| /|\mu \delta \rho| \sim\left(k v_{\mathrm{f}, 0}\left\langle t_{\mathrm{s}}\right\rangle / \mu\right)^{1 / 2}$ in the mid- $k$ regime, and $\left|\delta \rho_{\mathrm{d}}\right| /|\mu \delta \rho| \sim\left(k v_{\mathrm{f}, 0}\left\langle t_{\mathrm{s}}\right\rangle / \mu^{2}\right)^{1 / 3}$ in the high- $k$ regime. At lower $k$, the deviation from the simple structure above is more pronounced (owing to transverse components of $\mathbf{w}_{\mathrm{s}}$ as well as non-zero $\tau$ ), but these are not essential to the mode dynamics.

Qualitatively, like the acoustic RDI, the gas density peak generated by the leading fast wave decelerates the dust, generating a 'pileup.' But this dust overdensity, in turn, pushes on the gas. Because the mode is traveling in the direction $\hat{\mathbf{k}}$ with a phase velocity matched to the dust drift in that direction, these effects add coherently (if we imagine moving with a Lagrangian dust 'patch'), generating rapid growth of the instability.

(ii) Slow-wave RDI: Here the phase velocity is that of a slow wave $\mathbf{v}_{\mathrm{p}} \approx \mathbf{v}_{-}$, and again, at short wavelengths the gas perturbation closely resembles a perturbed slow-magnetosonic wave. The dust velocity perturbations $\delta \mathbf{v}$ are close to in-phase with $\delta \mathbf{u}$ (with a somewhat smaller amplitude), and both $\delta \mathbf{v}$ and $\delta \mathbf{u}$ are primarily confined to the $\hat{\mathbf{B}}_{0}-\hat{\mathbf{k}}$ plane. The phase offset between dust and gas density perturbations is similar to the fast-magnetosonic RDI $\left(\sim 30^{\circ}\right)$ but has opposite sign, generating an even stronger proportional dust-density fluctuation, with $\delta \rho_{\mathrm{d}} / \mu \delta \rho$ scaling similar to the acoustic/fast case but with an extra power $\sim\left(w_{\mathrm{s}} / v_{\mathrm{f}, 0}\right)^{-1 / 4}$ when $w_{\mathrm{s}} \ll v_{\mathrm{f}, 0}$. The details therefore differ, but the qualitative scenario is quite similar to the fast mode.

(iii) Alfvén-Wave RDI: The phase velocity is that of an Alfvén wave $\mathbf{v}_{\mathrm{p}} \approx \mathbf{v}_{\mathrm{A}}$, and at high $k$ the gas perturbation also resembles a perturbed Alfvén wave. For the fastest-growing mode, usually in the $\hat{\mathbf{B}}_{0}-\mathbf{w}_{s}$ or $\hat{x}-\hat{z}$ plane, the gas perturbation is primarily an incompressible mode with $\delta \mathbf{u}$ and $\delta \mathbf{B}$ in the $\hat{y}$ direction ( $180^{\circ}$ out-of-phase). The dust introduces some weak compressibility to the gas (and $\delta \mathbf{u}$ and $\delta \mathbf{B}$ terms in the $\hat{x}-\hat{z}$ plane) but these are small if $\mu \ll 1$ (suppressed by $\sim \mu$ ). The dust velocity perturbation $\delta \mathbf{v}$ is also primarily in the same direction $(\hat{y})$ with somewhat smaller amplitude than $\delta \mathbf{u}$ (leading $\delta \mathbf{u}$ by $\sim 30^{\circ}$ ); however, the compressive (non-transverse) components of the dust velocity perturbation are not strongly suppressed (they are smaller than $\delta \mathbf{v}_{y}$ by only a modest factor), so the dust still features a large-density perturbation $\delta \rho_{d}$. This, like the mode growth rate in the mid- $k$ regime, depends on the existence of non-vanishing Lorentz forces on dust, which couple the transverse B-field perturbations to longitudinal dust velocity perturbations: over much of the interesting parameter space, we can approximate $\left|\delta \rho_{\mathrm{d}}\right| \approx \mu \rho\left(\left|\delta B_{y}\right| / \sqrt{\rho c_{\mathrm{s}}^{2}}\right)\left[k c_{\mathrm{s}}\left\langle t_{\mathrm{L}}\right\rangle / \mu^{2}\right]^{1 / 3}$ (and note $\delta \rho_{\mathrm{d}}$ is out-of-phase with $\delta \mathbf{B}_{y}$ by $\sim 150^{\circ}$ ). So the perturbation is suppressed (like the growth rate) at low- $\tau$, and scales with $\delta \mathbf{B}$ via $t_{\mathrm{L}}, \mu$, and $w_{\mathrm{s}}$ (as opposed to $t_{\mathrm{s}}$ or $\beta$ ). In the high- $k$ limit, the velocity dependence of the drag law (parametrized through $\zeta_{w}$ ) is able to provide the necessary coupling of the transverse gas velocity perturbations to longitudinal dust perturbations, and the mode is still able to grow even if $\tau=0$.

Essentially, this instability amounts to a similar 'pileup' of dust pushing back on the gas, adding coherently because the phase velocity of the gas wave matches the dust drift in the same direction. However, the 'pushing' is mediated by the magnetic fields and Lorentz forces (the $\delta \mathbf{B}$ and dust fluctuations interacting), instead of gas pressure and aerodynamic/Coulomb drag.

\section{THE GYRO-RESONANCES}

\subsection{Overview}

In the previous section, we considered resonances between 'advective' dust mode(s) - i.e. a mode with frequency $\omega_{\mathrm{d}} \approx \mathbf{k} \cdot \mathbf{w}_{\mathrm{s}}-$ and the different gas modes (Alfvén, slow, and fast) with frequency $\omega_{\mathrm{g}}$. The resulting RDIs are unstable over a wide range of wavelengths. However, the dust is also affected by the magnetic field, which causes it to undergo gyro-motion, and the resonance between a gas mode and a dust gyro-mode leads to a new family of RDIs - the gyro-resonant RDIs.

More specifically, in addition to the undamped advection mode (with $\omega_{\mathrm{d}}=\mathbf{k} \cdot \mathbf{w}_{\mathrm{s}}$ ), there are three damped dust eigenmodes in equation (25). In general, all three depend on $\left\langle t_{\mathrm{L}}\right\rangle$ (or $\tau$ ), but if we consider large $\tau \gg 1$ (the case we will show is of relevance below) or special angles (e.g. where $\left.\cos ^{2} \theta_{\mathbf{B w}}=1\right)$, then they separate into $\omega_{\mathrm{d}} \approx \mathbf{k} \cdot \mathbf{w}_{\mathrm{s}}-i\left\langle t_{\mathrm{s}}\right\rangle^{-1}\left(\cos ^{2} \theta_{\mathbf{B w}}+\zeta_{w}\right)$ and $\omega_{\mathrm{d}} \approx \mathbf{k} \cdot \mathbf{w}_{\mathrm{s}} \pm\left\langle t_{\mathrm{L}}\right\rangle^{-1}-$ $i\left\langle t_{\mathrm{s}}\right\rangle^{-1}\left(3-\cos ^{2} \theta_{\mathbf{B w}}\right) / 2$. As explained in Appendix B, the first mode, with $\omega_{\mathrm{d}} \approx \mathbf{k} \cdot \mathbf{w}_{\mathrm{s}}-i\left\langle t_{\mathrm{s}}\right\rangle^{-1}\left(\cos ^{2} \theta_{\mathbf{B w}}+\zeta_{w}\right)$, leads to the high- $k$ regime of the standard RDI (see Section 5.1.1), but does not allow for any distinct resonances. Because it is damped, it can never exactly match the resonant condition with the gas, and so is only relevant once its imaginary part (the damping) is sufficiently small compared to other terms; i.e. at high $k$, once $\Im(\omega) \gtrsim\left\langle t_{\mathrm{L}}\right\rangle^{-1}$ (see Appendix. B2). This mode is present in the acoustic RDI as well (with $\cos ^{2} \theta_{\mathbf{B w}}=1$; see Paper I and Paper II).

However, now let us consider the $\omega_{\mathrm{d}} \approx \mathbf{k} \cdot \mathbf{w}_{\mathrm{s}} \pm\left\langle t_{\mathrm{L}}\right\rangle^{-1}-i\left\langle t_{s}\right\rangle^{-1}\left(3-\cos ^{2} \theta_{\mathbf{B w}}\right) / 2$ modes, which involve damped dust gyro-motion. For the same reason as above, these can only approximately satisfy the resonance condition when the damping term is small compared to the other terms, i.e. when $\left|\mathbf{k} \cdot \mathbf{w}_{\mathrm{s}} \pm\left\langle t_{\mathrm{L}}\right\rangle^{-1}\right| \approx \omega_{\mathrm{g}} \gg\left|\left\langle t_{\mathrm{s}}\right\rangle^{-1}\left(3-\cos ^{2} \theta_{\mathbf{B w}}\right) / 2\right|$. If also $\left|\mathbf{k} \cdot \mathbf{w}_{\mathrm{s}}\right| \gg\left\langle t_{\mathrm{L}}\right\rangle^{-1}$, then the $\left\langle t_{\mathrm{L}}\right\rangle^{-1}$ term is also sub-leading, 
the mode again reduces to an advection mode with $\omega_{\mathrm{d}} \approx \mathbf{k} \cdot \mathbf{w}_{\mathrm{s}}$, and we simply recover the high- $k$ limit of the standard MHD-mode RDIs. However, if $\left\langle t_{\mathrm{L}}\right\rangle^{-1} \gg\left|\mathbf{k} \cdot \mathbf{w}_{\mathrm{s}}\right|$ and $\left\langle t_{\mathrm{L}}\right\rangle^{-1} \gg\left|\left\langle t_{\mathrm{s}}\right\rangle^{-1}\left(3-\cos ^{2} \theta_{\mathbf{B w}}\right) / 2\right|$ (i.e. $\left.\tau \gg 1\right)$, the resonance condition becomes

$\omega_{\mathrm{d}}^{\text {gyro }} \approx \mathbf{w}_{\mathrm{s}} \cdot \mathbf{k} \pm\left\langle t_{\mathrm{L}}\right\rangle^{-1}= \pm\left(\mathbf{v}_{\mathrm{A}}, \mathbf{v}_{+}, \mathbf{v}_{-}\right) \cdot \mathbf{k}$.

This condition, which relies on the dust gyro-motion, has a fundamentally different character from the Alfvén and fast/slow RDIs above. There, if the resonant condition was satisfied at a given angle $\hat{\mathbf{k}}$, it was satisfied for all $k=|\mathbf{k}|$. For the gyroresonances, however, at a given angle or phase velocity $v_{\mathrm{p}}=\left|\mathbf{v}_{\mathrm{p}}(\hat{\mathbf{k}})\right|$ the condition is only satisfied around a particular $k$, namely $k^{-1} \approx \pm v_{\mathrm{p}}(\hat{\mathbf{k}})\left\langle t_{\mathrm{L}}\right\rangle$. The resonances are sharply peaked in $k$, with a specific maximum growth rate, owing to the fact that they involve a resonance with a mode of fixed physical frequency (here, the gyro frequency). This makes them much more akin to the Brunt-Väisälä RDI or the epicyclic RDIs studied in Paper I and in detail in Squire \& Hopkins (2017a).

\subsection{Resonant wavelengths and angles}

If one chooses a wave-family (Alfvén, slow, fast), so $v_{\mathrm{p}}(\hat{\mathbf{k}})=v_{\mathrm{A},+,-}(\hat{\mathbf{k}})$ and angle $\hat{\mathbf{k}}$, then it is trivial to solve equation (43) for the wavenumber $k$ at which the gyro-resonance will occur:

$k_{\text {gyro }}^{-1}=\left\langle t_{\mathrm{L}}\right\rangle\left|\mathbf{w}_{\mathrm{s}} \cdot \hat{\mathbf{k}} \pm v_{\mathrm{p}}(\hat{\mathbf{k}})\right|$

Equivalently, we can invert this to solve for the resonant angles $\hat{\mathbf{k}}=\hat{\mathbf{k}}_{\mathrm{gyro}}$ at a given $k=k_{\mathrm{gyro}}$. Since $\left|\mathbf{w}_{\mathrm{s}} \cdot \hat{\mathbf{k}} \pm v_{\mathrm{p}}(\hat{\mathbf{k}})\right| \leq w_{\mathrm{s}}+v_{\mathrm{f}, 0}$, at sufficiently low $k\left(k^{-1} \gtrsim\left\langle t_{\mathrm{L}}\right\rangle\left(w_{\mathrm{s}}+v_{\mathrm{f}, 0}\right)\right)$, equation (43) cannot be satisfied for any MHD wave family or angle, and no gyro-resonances exist. If $k_{\mathrm{gyro}}$ is sufficiently high, as noted above, equation (43) becomes $\mathbf{w}_{\mathrm{s}} \cdot \mathbf{k} \approx \omega_{\mathrm{g}}$ and the gyro-RDI modes are degenerate with the MHD-wave RDIs; this occurs when $\left|\mathbf{w}_{\mathrm{s}} \cdot \mathbf{k}\right| \sim v_{\mathrm{p}} k_{\mathrm{gyro}} \gg\left\langle t_{\mathrm{L}}\right\rangle^{-1}$, i.e. when $v_{\mathrm{p}} /\left|\mathbf{w}_{\mathrm{s}} \cdot \hat{\mathbf{k}} \pm v_{\mathrm{p}}\right| \gg 1$. Finally, if $\left|\mathbf{w}_{\mathrm{s}} \cdot \mathbf{k} \pm\left\langle t_{\mathrm{L}}\right\rangle^{-1}\right| \sim v_{\mathrm{p}} k_{\mathrm{gyro}} \lesssim\left(3-\cos ^{2} \theta_{\mathbf{B w}}\right) / 2\left\langle t_{\mathrm{s}}\right\rangle$ (if, e.g. the $\mathbf{w}_{\mathrm{s}} \cdot \mathbf{k}$ and $\left\langle t_{L}\right\rangle^{-1}$ terms cancel, so $\left|\omega_{\mathrm{g}}\right|$ is small), then the damping term $\left(-i\left\langle t_{\mathrm{s}}\right\rangle^{-1}\left(3-\cos ^{2} \theta_{\mathbf{B w}}\right) / 2\right.$ above) is not small compared to the other terms in the equation, and the RDI condition is not actually valid. Thus instability requires $v_{\mathrm{p}} /\left|\mathbf{w}_{\mathrm{s}} \cdot \hat{\mathbf{k}} \pm v_{\mathrm{p}}\right| \gtrsim\left(3-\cos ^{2} \theta_{\mathbf{B w}}\right) / 2 \tau$. These conditions can only be simultaneously satisfied when $\tau \gtrsim 1$. Therefore, even though equation (43) is actually four differently signed equations (each pair of \pm terms being independent) for each branch (Alfvén, slow, fast) of $\omega_{\mathrm{g}}$, not all of these produce interesting instabilities. Generally the 'interesting' gyro-RDI branches occur only when $\left\langle t_{\mathrm{L}}\right\rangle^{-1} \gtrsim\left|\omega_{\mathrm{g}}\right| \gtrsim\left\langle t_{\mathrm{s}}\right\rangle^{-1}$, reducing the number of interesting and unique gyro-RDI branches to 6 (two for each wave family corresponding to $v_{\mathrm{p}}(\hat{\mathbf{k}}) k \approx \pm\left\langle t_{\mathrm{L}}\right\rangle^{-1}$ ) at intermediate $k$.

For the fast-gyro RDI, the fact that $v_{\mathrm{p}} \approx v_{\mathrm{f}, 0}$ is only weakly dependent on angle means that the resonance condition is simple. For $\left|\mathbf{w}_{\mathrm{s}} \cdot \hat{\mathbf{k}}\right| \lesssim v_{\mathrm{f}, 0}$, the resonance condition can only be satisfied around a narrow range of wavenumbers: $k_{\mathrm{gyro}}^{-1} \approx\left\langle t_{\mathrm{L}}\right\rangle v_{\mathrm{f}, 0}$ (nearly independently of angle). For $\left|\mathbf{w}_{\mathrm{s}} \cdot \hat{\mathbf{k}}\right| \gg v_{\mathrm{f}, 0}$, one finds $k_{\text {gyro }}^{-1} \approx\left\langle t_{L}\right\rangle\left|\mathbf{w}_{s} \cdot \hat{\mathbf{k}}\right|$, so the 'resonant angle' is given by $\cos \theta_{\mathbf{w k}}=\hat{\mathbf{w}}_{\mathrm{s}} \cdot \hat{\mathbf{k}} \approx \pm 1 /\left(\left\langle t_{\mathrm{L}}\right\rangle w_{\mathrm{s}} k\right){ }^{19}$

The Alfvén-gyro RDI satisfies $v_{\mathrm{p}}=v_{\mathrm{A}} \cos \theta_{\mathbf{B k}}$, so if $\left|v_{\mathrm{p}}\right| \gtrsim\left|\mathbf{w}_{\mathrm{s}} \cdot \hat{\mathbf{k}}\right|$ then for all $k \geq 1 /\left(v_{\mathrm{A}}\left\langle t_{\mathrm{L}}\right\rangle\right)$, the resonant angles are given by $\cos \theta_{\mathbf{B k}}=\hat{\mathbf{k}} \cdot \hat{\mathbf{B}}_{0} \approx \pm 1 /\left(\left\langle t_{\mathrm{L}}\right\rangle v_{\mathrm{A}} k\right)$. If $\left|\mathbf{w}_{\mathrm{s}} \cdot \hat{\mathbf{k}}\right| \gg v_{\mathrm{p}}$, then the resonant angle is again just $\cos \theta_{\mathbf{w k}} \approx \pm 1 /\left(\left\langle t_{\mathrm{L}}\right\rangle w_{\mathrm{s}} k\right)$.

The resonant angles of the slow-gyro RDI are similar to the Alfvén case, but with the slow phase velocity; thus, approximately, we can simply take $v_{\mathrm{A}} \rightarrow \operatorname{MIN}\left(v_{\mathrm{A}}, c_{\mathrm{s}}\right)$ in the Alfvén expressions above.

Within the range of resonant angles, there is a fairly weak dependence of the growth rate on the particular angle chosen (or equivalently, on $k_{\text {gyro }}$ ), barring the pathological cases above (where e.g. $k_{\text {gyro }} \rightarrow \infty$ ). For this reason, we do not (as we did for the MHD-wave RDIs) attempt to estimate the fastest-growing resonant angle within the resonant branch.

\subsection{Growth rates and (in)stability conditions}

Owing to the presence of the damping term discussed above, and the set of four resonant equations, exact expressions for the growth rates of the gyro-RDI at, for example, high or low $k_{\text {gyro }}$, are even more opaque than for the MHD-wave RDIs. However, since $\tau \gtrsim 1$ is required anyway for interesting behavior of these modes, if we assume $\tau \gg 1$ and expand the dispersion relation, the relevant behaviors become more clear. $^{20}$

A straightforward, but tedious, direct analysis of the dispersion relation at high- $\tau$ shows that when the dust-to-gas ratio $\mu$ is small, instability typically requires $\mu^{1 / 2} \tau \gtrsim 1$ and $w_{\mathrm{s}} \sim v_{\mathrm{p}}$ (or at least $w_{\mathrm{s}}$ not too small compared to $v_{\mathrm{p}}$; otherwise, the damping terms in the dust eigenmode are not negligible (all $w_{\mathrm{s}}$ are unstable, however, if $\mu \gtrsim 1$ ). If these conditions are met, then the growth rates are approximately given by

$\Im\left(\omega_{\text {gyro }}\right) \approx\left|\hat{\mathbf{w}}_{\mathrm{s}} \cdot \hat{\mathbf{k}}\right| \frac{\mu^{1 / 2} k_{\text {gyro }} w_{\mathrm{s}}}{\left|k_{\mathrm{gyro}} w_{\mathrm{s}}\left\langle t_{\mathrm{L}}\right\rangle \pm 1\right|^{1 / 2}}=\left|\hat{\mathbf{w}}_{\mathrm{s}} \cdot \hat{\mathbf{k}}\right| \frac{\mu^{1 / 2}\left\langle t_{\mathrm{L}}\right\rangle^{-1}}{\left|1 \pm\left(1 \pm v_{\mathrm{p}}(\hat{\mathbf{k}}) / w_{\mathrm{s}}\right)\right|^{1 / 2}}$.

\footnotetext{
${ }^{19}$ Note when $\mathbf{w}_{\mathrm{s}} \cdot \mathbf{k} \approx v_{\mathrm{p}}(\hat{\mathbf{k}})$, the '+' branch of equation (44) just gives a similar solution to when $\mathbf{w}_{\mathrm{s}} \cdot \mathbf{k} \neq v_{\mathrm{p}}(\hat{\mathbf{k}})$, while the '-' branch nearly cancels the two and produces very large $k_{\text {gyro }}$. This, however, is just the limit where the gyro-RDI becomes degenerate with the MHD-wave RDI.

${ }^{20}$ Expanding at high- $\tau$, assuming $k_{\mathrm{gyro}} \propto \omega \propto\left\langle t_{\mathrm{L}}\right\rangle^{-1} \propto \tau$ to leading order, we obtain the dispersion relation $0=\tilde{\omega}^{2} \beta_{i}\left[\left\{1-\left(\tilde{\omega}-a_{w}\right)^{2}\right\}\left(\tilde{\omega}^{2}-a_{\mathrm{B}}^{2}\right)\left(\tilde{\omega}^{2}-a_{\mathrm{B}}^{2} \hat{k}_{z}^{2}\right)+\right.$ $\left.\mu\left(\tilde{\omega}-a_{w}\right)^{2}\left\{2 \tilde{\omega}^{2}+\mu\left(\tilde{\omega}-a_{w}\right)^{2}-a_{\mathrm{B}}^{2}\left(1+\hat{k}_{z}^{2}\right)\right\}\right]+a_{\mathrm{B}}^{2}\left[\left\{\left(\tilde{\omega}-a_{w}\right)^{2}-1\right\}\left(\tilde{\omega}^{2}-a_{\mathrm{B}}^{2} \hat{k}_{z}^{2}\right)^{2}-\mu\left(\tilde{\omega}-a_{w}\right)^{2}\left\{\left(1+\hat{k}_{z}^{2}\right)\left(\tilde{\omega}^{2}-a_{\mathrm{B}}^{2} \hat{k}_{z}^{2}\right)+\mu \hat{k}_{z}^{2}\left(\tilde{\omega}-a_{w}\right)^{2}\right\}\right], \quad$ where $\tilde{\omega} \equiv \omega\left\langle t_{\mathrm{L}}\right\rangle, a_{w} \equiv \mathbf{w}_{\mathrm{S}} \cdot \mathbf{k}_{\mathrm{gyro}}\left\langle t_{\mathrm{L}}\right\rangle$, and $a_{\mathrm{B}} \equiv v_{\mathrm{A}} k_{\mathrm{gyro}}\left\langle t_{\mathrm{L}}\right\rangle$.
} 
So the fastest-growing gyro modes will tend to be those aligned with the dust $\operatorname{drift}\left(\hat{\mathbf{w}}_{\mathrm{s}} \cdot \hat{\mathbf{k}}= \pm 1\right)$, but a wide range of angles and resonant $k_{\text {gyro }}$ will have growth rates $\Im(\omega) \sim \mu^{1 / 2} /\left\langle t_{\mathrm{L}}\right\rangle$. Equation (45) provides a reasonable approximation to the peak growth rates of the gyro modes in Figs 2-5.

We stress that the instability requirements above are not a strong limit, but an approximate guide; there often exist mode angles where the gyro-RDIs are unstable despite significantly smaller $w_{\mathrm{s}}$ or $\mu$ (evident in the slow-wave and Alfvén-wave resonance cases in Fig. 2). For example, if we expand in both large- $\tau$ and small- $\mu$, assuming $\omega \sim \mathcal{O}(\tau)+\mathcal{O}\left(\mu^{1 / 2} \tau\right)+\cdots$, we find that at the angle $\hat{\mathbf{w}}_{\mathrm{s}} \cdot \hat{\mathbf{k}}_{=} \pm\left(k_{\text {gyro }}\left|w_{\mathrm{s}}-v_{\mathrm{A}}\right|\left\langle t_{\mathrm{L}}\right\rangle \mid\right)$, the term that usually stabilizes the instability at small- $\mu$ vanishes,. This causes the gyro-RDI modes to be unstable so long as $w_{\mathrm{s}} \geq v_{\mathrm{A}}$ or $w_{\mathrm{s}} \geq$ $v_{\mathrm{A}} / 2$ (depending on which solution branch we consider), albeit with slightly different growth rates, $\Im\left(\omega_{\text {gyro }}\right) \approx \mu^{1 / 2}\left\langle t_{\mathrm{L}}\right\rangle^{-1}\left(w_{\mathrm{s}} / v_{\mathrm{A}}-1\right)^{1 / 2} / 2$ or $\Im\left(\omega_{\text {gyro }}\right) \approx \mu^{1 / 2}\left\langle t_{\mathrm{L}}\right\rangle^{-1}\left(2 w_{\mathrm{s}} / v_{\mathrm{A}}-1\right)^{1 / 2} / 2$.

\subsection{Mode structure}

To leading order, the dust perturbation in $\left(\delta \mathbf{v}, \delta \rho_{\mathrm{d}}\right)$ is incompressible gyro motion around $\hat{\mathbf{B}}_{0}$. The dust back-reaction produces a proportional $\delta \mathbf{B}$ perturbation $\left(|\delta \mathbf{B}| \sim(\rho / \beta)^{1 / 2}|\delta \mathbf{v}|\right)$ which lags $\delta \mathbf{v}$ by a modest $\left(\sim 30 \rightarrow 50^{\circ}\right)$ phase offset. This and the drag terms drive the gas into phase-lagged gyro motion and generate a compressible component (non-vanishing $\delta \rho_{\mathrm{d}}$ and $\delta \rho$ ), but these are suppressed by (roughly) $\sim \mu^{1 / 2}$ and $\sim \mu$, respectively.

However, the phase velocity is strongly modified from the pure gyro case (where it scales as $\mathbf{w}_{\mathrm{s}} \cdot \mathbf{k} \pm\left\langle t_{\mathrm{L}}\right\rangle^{-1}$ ). It scales in a fairly complicated manner, but is of order the growth rate (smaller by another power of $\sim \mu^{1 / 4}$, very approximately).

\section{OTHER UNSTABLE MODES}

Recall the dispersion relation is 10th-order; at any given $\mathbf{k}$, there are typically $\sim 3-7$ unstable (growing) modes (i.e. branches of the dispersion relation). We will discuss the additional unstable modes only very briefly, because they either (a) have much smaller growth rates than those we discussed above or (b) only appear in pathological situations.

The additional modes include analogues of the out-of-resonance 'intermediate' and 'slow' modes from Paper II; these are modes with phase velocities that (approximately) satisfy equation (27), i.e. $v_{\mathrm{p}}=\left(\mathbf{w}_{\mathrm{s}} \cdot \hat{\mathbf{k}}, \pm v_{\mathrm{A}}, \pm v_{\mathrm{f}}, \pm v_{\mathrm{s}}\right)$ (matching either the dust drift or the Alfvén/fast/slow-mode velocities at that $\mathbf{k}$ ). At resonance, the mode satisfies several of these phase-velocity conditions at the same time, so a subset of the modes becomes degenerate and the growth rates become much larger. However, even out of resonance, for any mode that approximately solves the gas equation without dust, the additional corrections from the dust-gas-coupling usually lead to a positive growth rate - i.e. the modes obey $\omega \approx v_{\mathrm{p}} k+i \mathcal{O}(\mu \ldots)$, where the growth rate is small but positive and non-zero (and usually scales as $\sim \mu$ for $\mu \ll 1$, as expected from non-degenerate perturbation theory). Some of these are illustrated in Fig. 2, particularly in the bottom-right 'no resonance' panel. Similarly, in Figs 4 and 5, we see that a broad range of angles away from resonance are still unstable. As discussed in Paper II for the acoustic RDI, while certain combinations of the parameters $\zeta_{s}, \zeta_{w}, \zeta_{q}$ can stabilize a subset of these modes out-of-resonance, there is invariably a different subset that is destabilized at the same time.

There also exist some growing modes that are not directly related to the 'natural' response of the un-coupled system (i.e. the uncoupled gas or dust modes), but these have very low growth rates at all $k$ (e.g. they are often suppressed by a factor of $\sim \mu k /\left(1+\tau^{2}\right)$ at small $k$ ).

Finally there are modes that can have very large growth rates but appear only for pathological parameter choices. For example, the 'decoupling' mode from Paper II is present here, if $\zeta_{w}<-1$ (at low- $\tau$; at high- $\tau$ the requirement is approximately $\zeta_{w} \cos ^{2} \theta_{\mathbf{B w}}<-1$ ). This large, negative $\zeta_{w}$ means that as the relative dust-gas velocity increases, the total force between dust and gas rapidly becomes weaker. Thus if the dust begins to accelerate relative to the gas, the coupling becomes weaker and the two rapidly separate (formally the growth rate is $\sim\left|\tilde{\zeta}_{w}\right| /\left\langle t_{\mathrm{s}}\right\rangle$ at all $k$ ). But this physically is unlikely: as shown in Paper II, while the scaling of $\zeta_{w}$ for Coulomb drag with super-sonic $\mathbf{w}_{\mathrm{s}}$ formally produces this instability, in that limit one should represent the drag via the sum of Coulomb and aerodynamic drag. The aerodynamic term (which becomes more tightly-coupled with higher drift velocity) will always dominate at large drift velocities. There are analogous instabilities that can appear with sufficiently negative $\zeta_{\mathrm{s}}$ or $\zeta_{q}$, but we do not expect any physical dust-gas couplings to produce such values.

\section{SCALES WHERE OUR DERIVATIONS BREAK DOWN}

\subsection{Largest wavelengths/timescales}

As discussed in Paper II in detail, the scalings and derivations presented here are valid over some range of spatial and timescales. At sufficiently long wavelengths (low- $k$ ), the wavelengths $\lambda \sim 2 \pi / k$ become comparable to some global gradient scale-length $L_{0}$ of the system, so a global solution (with appropriate boundary conditions) is obviously needed. However, as shown in Paper II, stratification of the background does not alter the character of the modes here, so long as $k \gg L_{0}^{-1}$; i.e. so long as we consider wavelengths short compared to the scale-length (we showed this in Paper II for the acoustic RDI, but since the dimensional scaling of the growth rates and mode structure here is similar, the qualitative conclusions are identical). Likewise, if the mode growth time is comparable to (or longer than) the global evolution time of the system $t_{0}$, a global solution is needed. The relevant global evolution times can include, e.g. the timescale for the dust to drift 'through' some global scale-length $L_{0}, t_{0} \sim L_{0} / w_{\mathrm{s}}$. Obviously, these scales are problem-specific; we discuss them in various contexts of astrophysical 

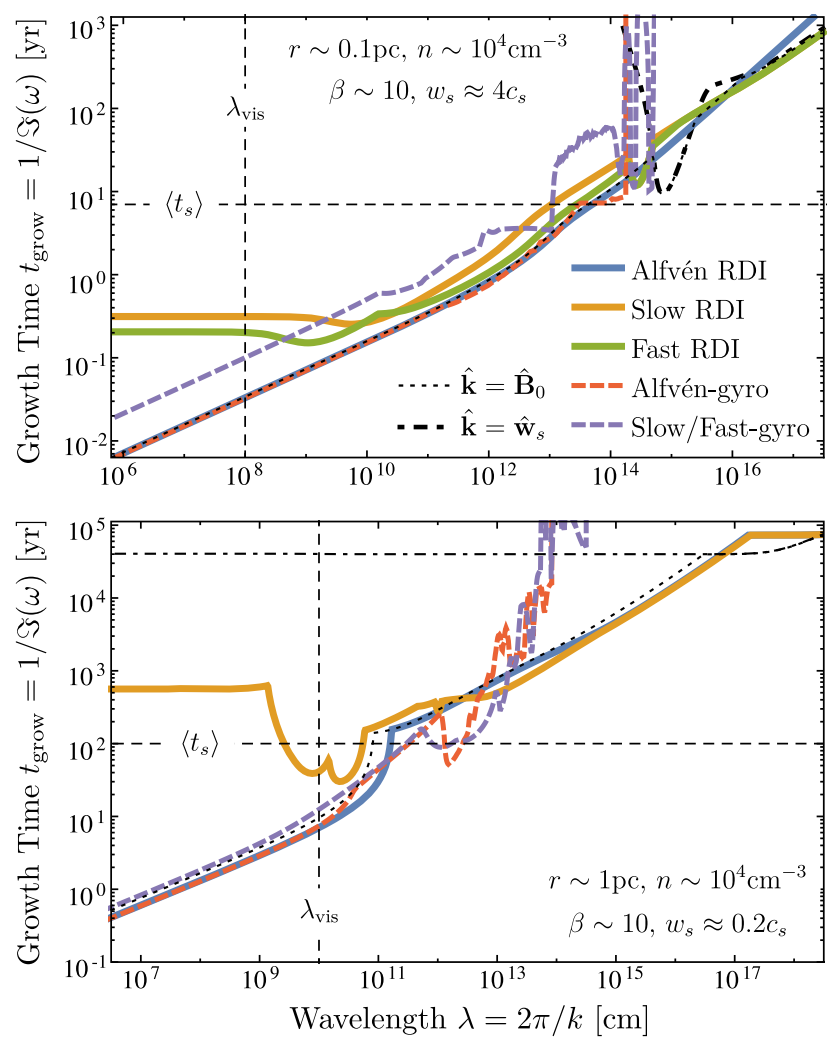

Figure 7. Growth timescale $t_{\text {grow }}=1 / \Im(\omega)$ of the RDI for two illustrative examples in an H II region (see Section 9.2.3). Top: Parameters (labeled) appropriate near $(r \sim 0.1 \mathrm{pc})$ the star(s), where the drift is supersonic $\left(w_{\mathrm{s}} \approx 4 c_{\mathrm{s}}\right)$. Bottom: Parameters appropriate further $(r \sim 1 \mathrm{pc})$ from the source(s) with subsonic $\operatorname{drift}\left(w_{\mathrm{s}} \approx 0.2 c_{\mathrm{s}}\right)$. For each line, we show the fastest-growing mode angle $(\hat{\mathbf{k}})$ that satisfies the relevant RDI condition at each $\lambda=2 \pi /|\mathbf{k}|$. We compare the Alfvén-wave, slow-wave, and fast-wave RDIs (fast only unstable when $w_{\mathrm{s}}>v_{\mathrm{f}, 0}$ ), Alfvén-gyro and slow/fast-gyro RDIs, and modes with $\hat{\mathbf{k}}=\hat{\mathbf{w}}_{\mathrm{s}}$ or $\hat{\mathbf{k}}=\hat{\mathbf{B}}_{0}$. In this plot, we also include the parallel (Braginskii) viscosity in the momentum equation $\left(\partial_{t} \mathbf{u}=\cdots+c_{s} \lambda_{\operatorname{mfp}} \nabla \cdot[\hat{\mathbf{B}} \hat{\mathbf{B}}(\hat{\mathbf{B}} \hat{\mathbf{B}}: \nabla \mathbf{u})]\right)$, which damps field-parallel motion below the viscous scale $\lambda_{\text {vis }} \sim \lambda_{\text {mfp }}$ (see Section 8 ). This decreases the growth rates of the slow- and fast-wave RDIs for $\lambda \lesssim \lambda_{\text {vis }}$, without affecting the Alfvén-wave RDI, which involves only perpendicular motions of the gas. In this example, at scales $\lambda \sim r$, the RDI growth time can be much shorter than $\mathrm{H}$ II region expansion times; while at short wavelengths within $r \lesssim 0.1 \mathrm{pc}$, growth timescales of the Alfvén RDI are as small as $\sim$ days!

applicability below (Section 9). Moreover, as discussed in Paper I and Squire \& Hopkins 2017a, if there are additional terms on large scales that need to be included (e.g. stratification, or centrifugal/coriolis forces, etc.) these almost always introduce new RDIs (even if they are unconditionally stable in a pure-gas medium), which may have faster growth rates at the largest wavelengths.

\subsection{Smallest (dissipation) scales}

At sufficiently small wavelengths (high- $k$ ), dissipative effects in the gas (viscosity, conductivity etc.) will become important. A necessary condition for our results to be valid is that the gas wave associated with the specific RDI in question (i.e. the Alfvén, slow, or fast wave) is not strongly affected by such effects. In a primarily neutral/molecular gas, the viscosity (or conductivity) is approximately isotropic, and so all waves will presumably be similarly damped, thus damping the RDIs for wavelengths below the mean-free path scale, $\lambda_{\mathrm{mfp}} \sim$ $10^{15} \mathrm{~cm}\left(n_{\text {gas }} / 1 \mathrm{~cm}^{-3}\right)^{-1}$. However, in a magnetized gas with a high ionization fraction, when the mean-free path is larger than the ion gyro-radius, the viscosity and conductivity are much larger parallel to the magnetic field than perpendicular to it (Braginskii 1965). This implies that while the slow and fast waves are damped on scales below the mean-free path, $\lambda_{\text {mfp }} \sim 10^{12} \mathrm{~cm}\left(T / 10^{4} \mathrm{~K}\right)^{2}\left(n_{\text {gas }} / 1 \mathrm{~cm}^{-3}\right)^{-1}$, the Alfvén wave remains unmodified until its wavelength approaches ion gyro-radius scales, $\left.\lambda_{\text {ion, gyro }} \sim 10^{7} \mathrm{~cm}^{(} n_{\text {gas }} / 1 \mathrm{~cm}^{-3}\right)^{-1 / 2} \beta^{1 / 2}$. This suggests that in hot ionized plasmas, the Alfvén-wave RDIs (both the standard and gyro-resonant variants) can survive unmodified on scales many orders of magnitude smaller than that where the slow and fast RDIs are damped by viscosity. This is illustrated in Fig. 7, which shows the numerically calculated RDI growth rates for parameters typical of an $\mathrm{H}$ II region (see Section 9.2.3), including the effect of a parallel (Braginskii) viscosity, $c_{\mathrm{s}} \lambda_{\mathrm{mfp}} \nabla \cdot[\hat{\mathbf{B}} \hat{\mathbf{B}}(\hat{\mathbf{B}} \hat{\mathbf{B}}: \nabla \mathbf{u})]$, in the momentum equation. We see that the while the slow and fast RDIs depart from their ideal scaling for $\lambda \lesssim \lambda_{\text {mfp }}$, the Alfvén-wave RDI is unaffected because it involves only perpendicular gas motions (aside from very small, $\sim \mu$, compressive corrections due to the dust; see Section 5.5).

Another small-scale limit on our treatment arises because the fluid approximation for the dust will break down on scales comparable to the mean grain spacing, $\lambda_{\mathrm{d} \text {,space }} \sim 6000 \mathrm{~cm}\left(R_{d} / 0.1 \mu \mathrm{m}\right)\left(n_{\text {gas }} / 1 \mathrm{~cm}^{-3}\right)^{-1 / 3}(\mu / 0.01)^{-1 / 3}$. This is extremely small compared to the viscous (mean-free path) scales, and even (usually) the gyro-radius scales. 


\subsection{Non-ideal effects}

Non-ideal MHD effects can similarly cause our treatment to be invalid if ionization fractions $\left(f_{\text {ion }}\right)$ are sufficiently low. In the highdensity, low- $f_{\text {ion }}$ (strong coupling) limit, one can parametrize the dominant effects as additional terms in the induction equation: $\partial \mathbf{B} / \partial t=$ $\nabla \times(\mathbf{u} \times \mathbf{B})-\nabla \times\left(\eta_{\text {Ohm }} \mathbf{J}\right)-\nabla \times\left(\eta_{\text {Hall }} \mathbf{J} \times \hat{\mathbf{B}}\right)-\nabla \times\left[\eta_{\mathrm{AD}} \hat{\mathbf{B}} \times(\mathbf{J} \times \hat{\mathbf{B}})\right]$, where $\mathbf{J}=\nabla \times \mathbf{B}$ is the current density and $\eta_{\text {Ohm }}, \eta_{\text {Hall }}$, and $\eta_{\mathrm{AD}}$ are the effective diffusivities for Ohmic resistivity, the Hall effect, and ambipolar diffusion, respectively. Ohmic resistivity will damp the slow and Alfvén modes, and modify the fast mode into a sound wave, above a wavenumber $k_{\text {Ohm }} \sim v_{p} / \eta_{\text {Ohm }}$. The Hall effect is not diffusive but modifies the Alfvén and slow wave dispersion relations for $k \gtrsim k_{\text {Hall }} \sim v_{p} / \eta_{\text {Hall }}$ into a shear-Alfvén branch with $\omega_{\mathrm{g}} \sim$ constant and a Whistler branch with $\omega_{\mathrm{g}} \propto k^{2}$. This in turn modifies the Alfvén and slow RDIs into two new RDI families (see Squire \& Hopkins 2017a). However, for all astrophysical contexts in Section 9 (except protoplanetary discs and planetary atmospheres) both $k_{\text {Ohm }}$ and $k_{\text {Hall }}$ correspond to wavelengths many orders of magnitude smaller than the other dissipative scales above. ${ }^{21}$ Likewise, effects of the current carried by grains themselves producing violations of ideal MHD are generally negligible. ${ }^{22}$

Ambipolar diffusion is more ambiguous: in primarily ionized gas it is negligible (the neutrals simply add to the effective weight of the ions). In primarily neutral gas (in the strong-coupling limit), it damps the Alfvén wave, but leaves the fast wave and $\hat{\mathbf{B}}$-parallel slow wave largely un-modified, while damping the slow wave (and modifying the fast wave to a sound wave) at field-perpendicular angles when $k \gtrsim$ $k_{\mathrm{AD}} \sim v_{\text {slow }} / \eta_{\mathrm{AD}}$ (Balsara 1996). So the slow-RDI modes will be stabilized at large- $k$ if $w_{\mathrm{s}} / v_{\mathrm{f}, 0}$ is sufficiently small (since the only resonant angles are nearly-perpendicular), while the fast-RDI modes are similar to those here ${ }^{23}$ (see also Squire \& Hopkins 2017a).

Finally, it is worth noting that in a well-ionized plasma, variants of Alfvén RDIs could be unstable even below the ion gyro-scale, so long as there exist nearly undamped waves (e.g. kinetic-Alfvén-wave or Whistler-wave RDIs). Given the complexity of the dispersion relations of even the simplest kinetic plasma waves, and the very small scales on which such instabilities would be expected to operate, we shall not consider this further here.

\subsection{Random (micro-physical) grain motions}

Our fluid approximation for the dust has assumed zero dust pressure (or temperature), viz., we assume that, in equilibrium, all dust particles with a given size, charge, and in a given (local) spatial location move with the same local equilibrium velocity through the gas. As discussed in Paper I, a finite dust pressure, if it exists, causes the dust eigenmodes (in a fixed gas background) to be weakly damped because the dust-density eigenmode couples back to the dust bulk velocity (see Appendix B). The damping rate is $\Im\left(\omega_{0, \text { dust }}\right) \sim-c_{\mathrm{s}, \mathrm{d}}^{2} k^{2}\left\langle t_{\mathrm{s}}\right\rangle$, where $c_{\mathrm{s}, d}$ is the speed of the local random motions in the dust. For the same reason that the gyro-resonant mode is unstable only once $\Im(\omega) \gtrsim\left\langle t_{\mathrm{s}}\right\rangle^{-1}$ (see Section 6 and Appendix B1), an ideal RDI mode with growth rate $\Im(\omega)$ (as discussed through Sections 3.4-6) will be modified when $\Im(\omega)\left\langle t_{\mathrm{s}}\right\rangle \lesssim\left\langle t_{\mathrm{s}}\right\rangle\left|\Im\left(\omega_{0, \text { dust }}\right)\right| \sim\left(c_{\mathrm{s}, \mathrm{d}} / c_{s 0}\right)^{2}\left(k c_{\mathrm{s}}\left\langle t_{\mathrm{s}}\right\rangle\right)^{2}$. Note that the RDI modes will not necessarily be damped when this is the case, particularly at high $\tau$; for instance, cosmic-ray instabilities (see Section 4.3) are well-known to be unstable for more realistic and complex cosmic-ray distributions (in fact, there are many more unstable modes than studied here; see e.g. Kulsrud \& Pearce 1969; Wentzel 1969).

There are two general causes for random dust motions that produce an effective 'dust pressure': (i) instabilities acting with growth times shorter than $t_{\mathrm{s}}$, in which case they can grow before the system actually reaches the equilibrium discussed in Section 3.1 (see e.g. Fig. 1 and Section 4.3) or (ii) 'dust microphysics.' The latter includes a number of effects not contained in our simple drag model (see e.g. Draine 2004). For example, the effect of dust Brownian motion is straightforward to estimate and generally negligible: the root-mean-squared dust velocity is $c_{\mathrm{s}, \mathrm{d}} \sim 3 k_{B} T / m_{\mathrm{dust}}$, giving $\left(c_{\mathrm{s}, \mathrm{d}} / c_{\mathrm{s} 0}\right)^{2} \sim 10^{-9}\left(R_{\mathrm{d}} / 0.1 \mu \mathrm{m}\right)^{-3}$. This low value implies that the RDIs will be affected only for very subsonic drift velocities or low $\mu$ (such that $\Im(\omega)\left\langle t_{\mathrm{s}}\right\rangle$ is very small anyway), very small scales (such that $k^{2} c_{\mathrm{s}}^{2}\left\langle t_{\mathrm{s}}\right\rangle^{2}$ is very large), or very small grains. Thus, other effects (e.g. gas viscosity, see above) will almost invariably be important before the Brownian motion of the grains becomes important. Other relevant dust microphysics includes photoelectric emission and photodesorption of atoms from the dust grains, which would cause random grain motions in a radiation field with a significant isotropic component. The magnitude of these effects will depend on complicating factors such as the grain shape and molecular structure (see Weingartner \& Draine 2001b), but will in general be more important for smaller grains, because a single electron or atom provides a proportionally larger kick to a smaller grain.

${ }^{21}$ Again in the low-ionization-fraction, high-density limit, we have $\eta_{\mathrm{Ohm}} \approx c^{2} m_{e} n_{t}\langle\sigma v\rangle_{e} /\left(4 \pi e^{2} n_{e}\right), \eta_{\mathrm{Hall}} \approx B c /\left(2 \pi^{1 / 2} e n_{e}\right)$, and $\eta_{\mathrm{AD}} \approx B^{2}\left(m_{\mathrm{n}}+\right.$ $\left.m_{\mathrm{i}}\right) /\left(\langle\sigma v\rangle_{\mathrm{i}} m_{\mathrm{i}} m_{\mathrm{n}} n_{\mathrm{i}} n_{\mathrm{t}}\right)$ where $m_{\mathrm{e}, \mathrm{in}, \mathrm{n}}$ are the electron/ion/neutral effective masses, $n_{\mathrm{e}, \mathrm{i}, \mathrm{t}}$ the free electron/ion/ion+neutral number densities, and $\langle\sigma v\rangle_{e, i}$ the electron/ion-neutral collision rates. If we take typical scalings in primarily neutral (molecular/atomic) gas, this gives $k_{\mathrm{Ohm}} \sim 10^{20}\left(v_{\mathrm{f}, 0}\left\langle t_{\mathrm{s}}\right\rangle\right)^{-1} f_{\mathrm{ion}}\left(n / \mathrm{cm}^{-3}\right)^{-1}$ and $k_{\text {Hall }} \sim 10^{16}\left(v_{\mathrm{f}, 0}\left\langle t_{\mathrm{s}}\right\rangle\right)^{-1} f_{\text {ion }} \beta^{1 / 2}\left(n / \mathrm{cm}^{-3}\right)^{-1 / 2}$.

${ }^{22}$ The equations solved here (equation 1 ) are valid if either (a) the timescale $t_{\text {charge }}$ for a grain to reach local charge-exchange equilibrium with the plasma is short compared to the drag timescale $t_{\mathrm{s}}$, and/or (b) the total charge carried by dust is negligible compared to that in ions/free electrons $\left(n_{\text {grain }}\left|q_{\text {grain }}\right| \ll e n_{e}\right)$. Otherwise, we need to account for the current carried by grains themselves (e.g. modifying $\mathbf{u} \times \mathbf{B} \rightarrow\left[\left(n_{i} q_{i} \mathbf{u}+n_{\text {grain }} q_{\text {grain }} \mathbf{v}\right) \times \mathbf{B}\right] /\left(n_{e} e\right)$ in the induction equation). For the standard scalings of grain charge in Section 3.3.4, $n_{\text {grain }}\left|q_{\text {grain }}\right| /\left(n_{e} e\right) \sim 10^{-10}\left(T / 10^{4} \mathrm{~K}\right)(\mu / 0.01) \mathrm{f}_{\text {ion }}^{-1} \mathrm{R}_{0.1}^{-2}$, and (using the charging rates from Tielens 2005 and assuming Coulomb drag) $t_{\text {charge }} / t_{\mathrm{S}} \sim 10^{-6}\left(T / 10^{4} \mathrm{~K}\right) R_{0.1}^{-2}$, so the grain-current corrections are indeed negligible, unless either $f_{\text {ion }}$ is so low that non-ideal MHD effects would dominate anyway, or grains are so small $(\sim \AA)$ that they cannot be described by a drag law in the first place.

${ }^{23}$ Alternatively (but equivalently), if we do not make a one-fluid approximation for ambipolar diffusion, but assume Epstein drag in molecular gas with $v_{\mathrm{p}} \sim v_{\mathrm{f}, 0}$ (and $\sim 0.1 \mu \mathrm{m}$ grains), then if $f_{\text {ion }} \ll 10^{-6}\left(w_{\mathrm{s}} / \mathrm{km} \mathrm{s}^{-1}\right)(\mu / 0.01)$ (typical in dense GMC cores), the typical force (momentum flux) from ions on neutrals becomes much weaker than the forcing from the grains on neutrals, so we should essentially consider the RDI to be between the grains and neutral gas alone. The MHD-RDI then reduces to the un-damped acoustic RDI, eliminating the slow and Alfvén modes, while leaving the fast mode as a sound wave. 
In addition, realistic grains will be drawn from a broad size distribution, and feature variations in their chemical compositions and shapes as well. This will in turn produce a range of dust drift velocities, Larmor times, and charge (even at fixed size, if composition and shape vary). While not truly random, these may have a similar effect to an effective 'dust pressure' as above. They will likely 'smear out' the resonances involving $w_{\mathrm{s}}$ and $\tau$, potentially decreasing the maximum growth rates while increasing the range of conditions/angles where at least some sub-population of grains is near-resonance. However, it is also possible that new instabilities might appear in this regime, for example, three-fluid resonances between gas, 'small' grains, and 'large' grains (or some other grain sub-populations). Exploring this regime will likely require simulations accounting for realistic size and charge distributions.

\subsection{Non-linear effects}

If the system is strongly non-linear, or there is sufficiently sharp structure in the velocity or density fields, dust trajectories become selfintersecting and we cannot apply the fluid approximation to the dust. In this limit, numerical simulations must be used to integrate particle trajectories directly (as opposed to using a local fluid or 'terminal velocity' approximation, sometimes seen in the literature but invalid in this limit).

\section{ASTROPHYSICAL APPLICATIONS}

In this section, we consider applications of the instabilities described above to astrophysical systems. To make detailed predictions for observable or physical consequences, numerical simulations, which can explore the fully non-linear regime, will be necessary. However, it is useful to consider the relevant time and spatial scales, so as to evaluate whether the instabilities can grow efficiently, and if so, which instabilities and limits apply.

For brevity and convenience, we will introduce the following notation for this section: let the temperature $T_{x} \equiv T / 10^{x} \mathrm{~K}$ (so, e.g. $T_{4}=T / 10^{4} \mathrm{~K}$ ); likewise for the density $n_{x} \equiv n_{\text {gas }} / 10^{x} \mathrm{~cm}^{-3}$ and the grain size $R_{\mathrm{d}} \equiv R_{0.1} 0.1 \mu \mathrm{m}$. We will also define $\tilde{\sigma} \equiv \sqrt{1+w_{\mathrm{s}}^{2} / c_{\mathrm{s}}^{2}}$ (which interpolates between 1 for sub-sonic drift and $w_{\mathrm{s}} / c_{\mathrm{s}}$ for super-sonic drift), $f_{\text {ion }}=n_{\mathrm{ion}} / n_{\mathrm{tot}}$ is the ionization fraction of the gas, and $\tilde{U} \equiv(1 / 2)\left(\left|U_{\text {grain }}\right| /\right.$ volts $) /\left(T / 10^{4} \mathrm{~K}\right)$, which should be $\sim 1$ if $\left|U_{\text {grain }}\right| \approx\left|Z_{\text {grain }}\right| e / R_{\mathrm{d}}$ with the scalings we have adopted in Section 3.3 .4 for collisional charging (this convention follows Draine 2011, but we note the scaling of $U_{\text {grain }}$ in hot gas can be complicated by a number of additional physical processes; see e.g. Draine \& Salpeter 1979b; Weingartner \& Draine 2001c). Where necessary, we will adopt $\theta_{\mathbf{B a}} \sim 45^{\circ}$, since our conclusions are insensitive to the relative angle of $\mathbf{B}$ and $\mathbf{a}$ so long as it is not very close to exactly perpendicular.

\subsection{Relevant scalings}

First we outline some general scalings of use below.

\subsubsection{Stopping time $\left(\mathrm{t}_{\mathrm{s}}\right)$}

The stopping time (drag coefficient) $\left\langle t_{\mathrm{s}}\right\rangle$ is a critical parameter determining the drift velocity, relevant spatial scales for the different modes, and growth timescales. In most cases below Coulomb or Epstein drag dominates the drag force. Inserting physical values in the scalings from Section 3.3, we find

$$
\begin{aligned}
t_{\mathrm{s}}^{\text {Coulomb }} & \sim 10^{4} \mathrm{yr}\left(\frac{10^{4} \mathrm{~K}}{T}\right)^{1 / 2}\left(\frac{\mathrm{cm}^{-3}}{n_{\text {gas }}}\right)\left(\frac{R_{d}}{0.1 \mu m}\right) \frac{\tilde{\sigma}^{3}}{\tilde{U}^{2} f_{\text {ion }}} \\
t_{\mathrm{s}}^{\text {Epstein }} & \sim 30 \tilde{U}^{2} f_{\text {ion }} \tilde{\sigma}^{-4} t_{\mathrm{s}}^{\text {Coulomb }} .
\end{aligned}
$$

So, in largely ionized media with trans-sonic or sub-sonic drift, Coulomb drag should dominate, while in primarily neutral media $\left(f_{\text {ion }} \ll 0.1\right)$ and/or systems with super-sonic $\operatorname{drift}\left(w_{\mathrm{s}} \gtrsim 2 c_{\mathrm{s}}\right)$, Epstein drag will dominate.

\subsubsection{Spatial scales $(\mathrm{k})$}

A useful corresponding spatial scale (which allows us to estimate whether a given wavelength is in the low- $k$, mid- $k$, or high- $k$ regime) is given by some characteristic speed multiplied by $t_{\mathrm{s}}$. It is convenient to use $c_{\mathrm{s}}\left\langle t_{\mathrm{s}}\right\rangle$ for reference, both because we used it in Paper II and because it is better-known than some other possibilities, e.g. $w_{\mathrm{s}}\left\langle t_{\mathrm{s}}\right\rangle$. One finds:

$c_{\mathrm{s}} t_{\mathrm{s}}^{\text {Coulomb }} \sim 0.1 \mathrm{pc}\left(\frac{\mathrm{cm}^{-3}}{n_{\text {gas }}}\right)\left(\frac{R_{\mathrm{d}}}{0.1 \mu m}\right) \frac{\tilde{\sigma}^{3}}{\tilde{U}^{2} f_{\text {ion }}}$,

and we can also calculate $c_{\mathrm{s}} t_{\mathrm{s}}^{\text {Epstein }}=c_{\mathrm{s}} t_{\mathrm{s}}^{\text {Coulomb }}\left(t_{\mathrm{s}}^{\text {Epstein }} / t_{\mathrm{s}}^{\text {Coulomb }}\right) \sim 3 \mathrm{pc} \tilde{\sigma}^{-1} n_{0}^{-1} R_{0.1}$, when Epstein drag dominates. 


\subsubsection{Strength of Lorentz forces $(\tau)$}

If one considers an ionized medium with Coulomb drag dominating (or comparable to Epstein/Stokes drag), then

$\tau^{\text {Coulomb }} \sim 100\left(\frac{T}{10^{4} \mathrm{~K}}\right)\left(\frac{0.1 \mu \mathrm{m}}{R_{d}}\right)\left(\frac{\mathrm{cm}^{-3}}{n_{\text {gas }}}\right)^{1 / 2}\left(\frac{10}{\beta}\right)^{1 / 2} \frac{\tilde{\sigma}^{3}}{\tilde{U} f_{\text {ion }}}$.

We can also calculate this for the Epstein case $\left(t_{\mathrm{s}}=t_{\mathrm{s}}^{\text {Epstein }}\right)$, giving: $\tau^{\text {Epstein }} \sim 30 T_{2} \tilde{U}\left(R_{0.1} \tilde{\sigma}\right)^{-1}\left(n_{0} \beta / 10\right)^{-1 / 2}$. Given these results, in cold, dense gas (e.g. GMCs, planetary discs, circum-nuclear or circum-AGN regions in galaxies, and cool-star winds) we expect $\tau \ll 1$, but in the diffuse ionized ISM, CGM, and some dusty atmospheres, we expect $\tau \gg 1$.

\subsubsection{Drift velocities $\left(\begin{array}{ll}w & 0\end{array}\right)$}

The equilibrium drift velocities (ignoring the geometric corrections from Lorentz forces) are given by $\mathbf{w}_{0}=\mathbf{a}\left\langle t_{\mathrm{s}}\right\rangle /(1+\mu) \sim w_{0} \sim|\mathbf{a}|\left\langle t_{\mathrm{s}}\right\rangle$ (since $\mu$ is not large). If the drift is sub-sonic, we can neglect the dependence of $\left\langle t_{\mathrm{s}}\right\rangle$ on $\mathbf{w}_{\mathrm{s}}$ itself, giving $w_{0} \approx w_{0}^{*} \equiv|\mathbf{a}|\left\langle t_{\mathrm{s}}\left(\mathbf{w}_{\mathrm{s}}=0\right)\right\rangle$, where $\left\langle t_{\mathrm{s}}\left(\mathbf{w}_{\mathrm{s}}=0\right)\right\rangle$ is just the stopping time scaling above (Epstein or Coulomb from equation 46 , as appropriate), with $\tilde{\sigma}=1$. If $w_{0}^{*} \gg c_{\mathrm{s}}$, the drift is super-sonic; in this case Epstein drag dominates, and the true drift velocity is approximately given by $w_{0} \approx a_{\gamma}^{-1 / 4}\left(w_{0}^{*} c_{s}\right)^{1 / 2}($ where $a_{\gamma} \equiv 9 \pi \gamma / 128$ is defined in Section 3.3, and $w_{0}^{*}$ is evaluated for Epstein drag).

The characteristic acceleration $|\mathbf{a}|$ depends on the system; recall, $\mathbf{a}$ is the sum of any difference in acceleration experienced by the gas and the dust (other than that from drag itself). In some regimes it is dominated by gravity (if e.g. the gas is supported hydrostatically, since the dust cannot be), and in others by radiative acceleration (of the gas, in line-driven winds, or of the dust, in continuum absorption by grains). Other forces, some induced by external radiation (e.g. coherent photo-electric and photo-desorption effects as described in Weingartner \& Draine 2001b, or Poynting-Robertson ' $\mathrm{drag}{ }^{24}$ ), or hydrodynamic and other forces on gas (e.g. if the gas is accelerating or decelerating owing to pressure gradients or expanding explosions, or being driven by pressure from cosmic rays) are also important in some regimes.

It is useful to consider some order-of-magnitude estimates for the drift induced by gravity and radiation pressure on dust, as these are both common sources of drift. Noting that the gravitational acceleration $a_{\text {grav }} \sim G M / r^{2} \sim \pi G \Sigma_{\text {eff }}$ where $\Sigma_{\text {eff }}$ is an effective surface density (of all enclosed mass), we find,

$$
\frac{\left(w_{0}^{*}\right)^{\text {grav }}}{c_{\mathrm{s}}} \sim \begin{cases}0.006 R_{0.1} \Sigma_{500}\left(n_{0} T_{4} \tilde{U}^{2} f_{\text {ion }}\right)^{-1} & (\text { Coulomb) } \\ 30 R_{0.1} \Sigma_{500}\left(n_{0} T_{2}\right)^{-1} & \text { (Epstein) },\end{cases}
$$

where $\Sigma_{500} \equiv \Sigma_{\text {eff }} /\left(500 \mathrm{M}_{\odot} \mathrm{pc}^{-2}\right)$, and we have scaled the Epstein drag expression to the lower temperatures $T \sim 100 \mathrm{~K}$ where it typically applies (see below).

For radiation pressure on grains with some coherent incident flux $\mathbf{F}$, the acceleration is $a_{\text {rad }} \sim Q_{\text {abs }} F \pi R_{\mathrm{d}}^{2} /\left(c m_{\text {grain }}\right)$, where $Q_{\text {abs }}$ is a (spectrally averaged) absorption efficiency, $c$ the speed of light, and $m_{\text {grain }}=(4 \pi / 3) \bar{\rho}_{\mathrm{d}} R_{\mathrm{d}}^{3}$. Noting that $t_{\mathrm{s}} \propto \bar{\rho}_{\mathrm{d}} R_{\mathrm{d}} /\left(\rho c_{\mathrm{s}}\right)$ when $w_{\mathrm{s}}=0$, for both Epstein and Coulomb drag, we find $a_{\text {rad }} t_{\mathrm{s}} / c_{\mathrm{s}} \sim Q_{\mathrm{abs}}(F / c) /\left(\rho c_{\mathrm{s}}^{2}\right) \sim Q_{\mathrm{abs}} e_{\text {rad }} / e_{\text {thermal }}$, where $e_{\text {rad }} \equiv F / c$ is the (coherent) radiation energy density, and $e_{\text {thermal }} \equiv \rho c_{\mathrm{s}}^{2}$ is the thermal energy density. More accurately, we can estimate:

$$
\frac{\left(w_{0}^{*}\right)^{\mathrm{rad}}}{c_{\mathrm{s}}} \sim Q_{\mathrm{abs}} \frac{e_{\mathrm{rad}}}{e_{\text {thermal }}} \begin{cases}0.03 \tilde{U}^{-2} f_{\text {ion }}^{-1} & \text { (Coulomb) } \\ 1 & \text { (Epstein) } .\end{cases}
$$

For reference, for a flux $F=L /\left(4 \pi r^{2}\right)$ at distance $r=r_{\mathrm{pc}} \mathrm{pc}$, with total luminosity $L=L_{6} 10^{6} L_{\odot}$, we have $e_{\text {rad }} / e_{\text {thermal }} \sim$ $800 L_{6} n_{0}^{-1} r_{\mathrm{pc}}^{-2} T_{4}^{-1}$. For an incident flux peaked around a wavelength $\lambda_{\text {rad }}, Q_{\mathrm{abs}} \sim 1$ if $R_{d} \gg \lambda_{\text {rad }}$ (geometric absorption), and $Q_{\mathrm{abs}} \sim$ $\left(R_{d} / \lambda_{\text {rad }}\right)$ if $R_{d} \ll \lambda_{\text {rad }}$.

\subsubsection{Scaling coefficients $(\zeta)$}

The scaling coefficients $\zeta$ (given in detail in Section 3.3) are usually only order-unity corrections to the growth rate; however, certain terms (relevant for specific modes) can become small or nearly cancel (e.g. $\left.\zeta_{\mathrm{s}}-\tilde{\zeta}_{w}\right)$ in certain regimes.

If the drift is dominated by Coulomb drag, in the sub-sonic limit (the only regime where Coulomb drag can dominate), one finds $\zeta_{\mathrm{s}} \approx 1-1 /(2 \ln \Lambda)+\left(-1.4+1.9 \zeta_{\mathrm{C}}\right)(\gamma-1) \approx 0.96-1.4(\gamma-1)$, and $\tilde{\zeta}_{w} \approx 1-3 a_{\mathrm{C}} w_{\mathrm{s}}^{3} \approx 1$. If the drift is super-sonic, Epstein drag always dominates Coulomb, and we have $\tilde{\zeta}_{w}=\left(1+2 a_{\gamma}\left(w_{\mathrm{s}} / c_{\mathrm{s}}\right)^{2}\right) /\left(1+a_{\gamma}\left(w_{\mathrm{s}} / c_{\mathrm{s}}\right)^{2}\right) \approx 2$, and $\zeta_{\mathrm{s}} \approx 1$ (independent of the equation-of-state or $\mathbf{w}_{\mathrm{s}}$, to leading order). Note $1-\zeta_{\mathrm{s}}$ (as it appears in, e.g. equation 39) scales more accurately as $1-\zeta_{\mathrm{s}} \approx[192+(9 \pi-64) \gamma] /[128+$ $\left.9 \pi \gamma\left(w_{\mathrm{s}} / c_{\mathrm{s}}\right)^{2}\right] \approx 1.3(5.4 / \gamma-1)\left(w_{\mathrm{s}} / c_{\mathrm{s}}\right)^{-2}$ in this limit (see Paper II). Sub-sonic Epstein drag is occasionally also an important regime; in this case $\tilde{\zeta}_{w} \rightarrow 1$ and $\zeta_{\mathrm{s}} \rightarrow(\gamma+1) / 2$ as $w_{\mathrm{s}} \rightarrow 0$ (for Stokes drag, $\tilde{\zeta}_{w}, \zeta_{\mathrm{s}} \rightarrow 1,[\gamma-1] / 2$ ).

\footnotetext{
${ }^{24}$ For our purposes, Poynting-Robertson 'drag' is an external acceleration of the dust (and contributes to a, driving instability) rather than a 'true drag force' (like Coulomb or Epstein or Stokes drag, which appear in $t_{\mathrm{s}}$ ), because momentum is exchanged between the dust and the radiation field, not between the dust and the gas. Of course, coupled dust-radiation systems with any momentum exchange terms will be subject to their own RDIs, which we will explore in future work.
} 
For intermediate and large grains (as in most cases of interest) when collisional charging dominates, we expect the dust-charge (Lorentzforce) parameter $\zeta_{q} \approx \gamma-1$, while for sufficiently small grains $\zeta_{q} \approx 0$. The division occurs at grain size $R_{\mathrm{d}} \sim 6 \AA T_{4}^{-1}$, so the $\zeta_{q} \approx 0$ regime only occurs for interesting grain sizes when $T \lesssim 100 \mathrm{~K}$. If photo-electric charging dominates, we expect $\zeta_{q} \approx(\gamma-3) / 2$ unless the grains are maximally-charged (the incident UV flux exceeds $F_{\mathrm{pe}} \gtrsim 2 \times 10^{-5} n_{0} T_{4}^{-1 / 2} \mathrm{erg} \mathrm{s}^{-1} \mathrm{~cm}^{-2}$ ), in which case $\zeta_{q} \approx 0$.

\subsection{Application to different environments}

\subsubsection{The warm ionized and warm neutral interstellar medium}

In the 'warm' ISM, we have gas with densities $n_{\text {gas }} \sim 0.1 \rightarrow 1 \mathrm{~cm}^{-3}$, temperatures $T \sim 10^{4} \mathrm{~K}$, typical grain sizes $R_{d} \sim 0.01 \rightarrow 0.1 \mu \mathrm{m}, \beta$ $\sim 1 \rightarrow 100$, and $e_{\text {rad }} \sim e_{\text {thermal }}$ (also with typical gravitational and radiative accelerations of grains comparable to one another). Despite modest $\beta$, this gives $\tau \sim 100 \gg 1$. For the warm ionized medium (WIM, with $f_{\text {ion }} \sim 1$ ) Coulomb drag dominates Epstein drag, while for the warm neutral medium (WNM) the drag regime depends on the ionization fraction (Coulomb drag dominates for $f_{\text {ion }} \geq 0.03$, which is reasonable for the WNM). Assuming mostly ionized gas with modest $\beta$, we have $w_{\mathrm{s}} / v_{\mathrm{f}, 0} \sim w_{\mathrm{s}} / c_{\mathrm{s}} \sim 0.03 f_{\text {ion }}^{-1} e_{\text {rad }} / e_{\text {thermal }}$, so the typical drift velocities are expected to be modestly sub-sonic (a few tenths of $\mathrm{km} \mathrm{s}^{-1}$; for more detailed calculations that give a very similar result, see Weingartner \& Draine 2001b).

Since $w_{\mathrm{s}} \lesssim \operatorname{MIN}\left(v_{\mathrm{A}}, c_{\mathrm{s}}\right)$, we expect the dominant resonance of the RDI at intermediate and high- $k$ to be the slow-magnetosonic or Alfvén RDI (the fast-magnetosonic RDI will, in general, not be possible in the warm medium, except in regions near massive stars or other sources of strong local grain acceleration). Both Alfvén and slow modes have similar growth rates but the Alfvén mode may be slightly faster-growing at intermediate wavelengths. Under these conditions, all length scales are formally unstable to the RDI and related instabilities discussed here, from the viscous scale to the disc scale height (the effective 'Reynolds number' of the instabilities will be very large). We expect $c_{\mathrm{s}}\left\langle t_{\mathrm{s}}\right\rangle \sim 0.1 \mathrm{pc}\left(R_{0.1} / n_{0} f_{\text {ion }}\right)$, with $\mu \sim 0.01$, suggesting scales from the largest available (disc scale height) down to $\sim 1 \mathrm{pc}$ will be in the long-wavelength (low- $k$ ) regime, while scales from $\sim 1$ pc down to $\sim$ au will be in the mid-wavelength resonant regime.

Recall that the slow-mode resonance at high $\tau$ has lower growth rates through the mid- $k$ regime, owing to the projection of the drift along $\hat{\mathbf{B}}_{0}$ (which means resonant angles with slow wavespeeds, nearly-perpendicular to $\hat{\mathbf{B}}_{0}$, are also nearly perpendicular to $\hat{\mathbf{w}}_{\mathrm{s}}$ ). At long wavelengths ( $\lambda \gtrsim p c$ ), for this particular set of parameters ( $\tau$ in particular), the growth rate is only weakly dependent on scale (in part owing to coincidence of the overlap of the low- $k$ mode, gyro mode, and slow mid- $k$ mode, but primarily because the fastest-growing modes are the parallel quasi-drift and quasi-sound modes), with growth timescales $t_{\text {grow }} \sim 70 \mathrm{Myr} n_{0}^{-3 / 4}$. This is comparable to, or shorter than, dynamical times in the gas on the same scales. At smaller scales, pc $\gtrsim \lambda \gtrsim$ au, the growth rates scale as $t_{\text {grow }} \sim 0.8 \mathrm{Myr}(\lambda / 100 \mathrm{au})^{1 / 2} f_{\text {ion }}^{-2} n_{0}^{-3 / 4}$ (note the dependence on $w_{\mathrm{s}}, \tau$, and $t_{\mathrm{s}}$ is such that this is grain-size independent). Although this becomes longer than e.g. turbulent eddy turnover times in the ISM on smaller scales, at sufficiently small scales $\lambda \lesssim$ au, the high- $k$ Alfvén resonance appears, whose growth rate is enhanced rather than suppressed at high- $\tau$, giving growth timescales in this regime of $t_{\text {grow }} \sim 700 \mathrm{yr} R_{0.1} n_{0}^{-1 / 2}(\lambda / \mathrm{au})^{1 / 3}\left(\tilde{U} / f_{\text {ion }}^{2}\right)^{1 / 3}$. If the instability can persist down to the thermal dust gyro radii $\left(\sim R_{\odot}\left(R_{0.1} / n_{0}\right)^{1 / 2}\right.$, estimated assuming Brownian motion of the dust gives $m_{\text {dust }} \delta v_{\text {thermal }}^{2} \sim k_{B} T_{\text {gas }}$, so produces an effective 'dust pressure' that can damp modes below this scale), then the growth time at this scale is $t_{\text {grow }} \sim 100 \mathrm{yr} R_{0.1}^{7 / 6}\left(\tilde{U} / f_{\text {ion }} n_{0}\right)^{2 / 3}$. 25

These instabilities could have a wide range of consequences. They could drive sightline-to-sightline extinction-curve variations in the WNM and WIM (which have long been observed to be ubiquitous; see Schlafly et al. 2016 for a review), as grains are clumped on different scales in a size-dependent fashion (producing local variations in the size distribution). The smallest grains $\left(R_{0.1} \ll 1\right)$, at the smallest wavelengths $(\ll 0.1 \mathrm{au})$ approach growth timescales (hence likely variability timescales for the clumping in the non-linear regime) that become human observable ( $\ll 10 \mathrm{yr}$ ), and could be important for the well-known turbulent scintillation 'cascade' apparently seen in radio (for reviews, see Bhat et al. 2004; Haverkorn \& Spangler 2013). Dust-to-gas ratio fluctuations on intermediate scales $\left(\lambda \gg 10^{3}\right.$ au) could explain the well-studied order-of-magnitude (or larger) excess (relative to the ISM abundance) of large grains $\left(R_{\mathrm{d}} \gtrsim 0.1 \mu \mathrm{m}\right)$ observed in the solar neighborhood (see e.g. Krüger et al. 2015; Alexashov et al. 2016, and references therein). On larger scales, this may explain some tentative observational suggestions of systematic dust segregation across galactic scale height (Gontcharov 2016; Schlafly et al. 2016). Given the large-scale and strong angle-dependence with respect to the local magnetic field angles, this will almost certainly alter predictions for grain-field alignment and therefore polarization (and 'spinning dust'), and may be necessary to explain the observational fact that polarized dust emission obeys different directional statistics (particularly in the ratio of E to B modes) to those expected of the B-field in MHD turbulence, on scales of order the galactic disc height (Caldwell, Hirata \& Kamionkowski 2017, but see also Kandel, Lazarian \& Pogosyan 2017).

${ }^{25}$ In the WIM $\left(f_{\text {ion }} \sim 1\right)$, ideal MHD is an excellent approximation. In the WNM, if $f_{\text {ion }}$ and $w_{\mathrm{s}} / c_{\mathrm{s}}$ are both sufficiently small, ambipolar diffusion may not be negligible. However the low- $k$ modes of interest act identically on both neutrals and ions, so are unaffected by this. On scales au $\lesssim \lambda \lesssim 2 \pi / k_{\mathrm{AD}} \sim$ $0.2 \mathrm{pc} n_{0}^{-1}(\beta / 10)^{-1 / 2}\left(0.01 / f_{\text {ion }}\right)$, the mid- $k$ modes may be damped in the WNM if $w_{\mathrm{s}} \ll c_{\mathrm{s}}$. On scales $\lesssim$ au, the high- $k$ Alfvén resonance in the WNM is a resonance between ions/fields and dust, and actually has a growth time that becomes shorter than the ion-neutral coupling time, so proper treatment requires a three-fluid RDI. 


\subsubsection{The circum-galactic and inter-galactic medium}

The circum-galactic medium (CGM) and inter-galactic medium (IGM) are also clearly observed to be dust-laden (see e.g. Chelouche, Koester \& Bowen 2007; Ménard et al. 2010; Ménard \& Fukugita 2012; Peek, Ménard \& Corrales 2015; Baes \& Viaene 2016), and can exhibit a very broad range of properties.

Temperatures are in the range $T \sim 10^{5} \rightarrow 10^{7} \mathrm{~K}$ (clouds with temperatures $\sim 10^{4} \mathrm{~K}$ also exist in the CGM closer to galaxies, but the conditions in such clouds are expected to be closer to the WIM discussed above) ${ }^{26}$ The medium is largely ionized $\left(f_{\text {ion }} \sim 1\right)$, and low-density $\left(n_{\text {gas }} \sim 10^{-6} \rightarrow 10^{-2} \mathrm{~cm}^{-3}\right.$ ), so $\tau \sim 10^{4} \rightarrow 10^{7} \gg 1$, but with typical $\beta \sim 10^{2} \rightarrow 10^{4}$ (see Tumlinson, Peeples \& Werk 2017 , and references therein). However, we caution that the behavior of $\tilde{U}$ in this temperature range is highly uncertain (owing to factors such as electron emission from highly charged grains). In the classic models of Draine \& Salpeter (1979b), for example, silicate grains range from $\tilde{U} \sim 0.01-0.1$, and graphite grains from $\tilde{U} \sim 0.001-3$, for $T \sim 10^{5} \rightarrow 10^{7} \mathrm{~K}$, depending on the grain size, illuminating intensity field, and background density (note $2 \tilde{U}$ here is approximately equal to $\phi$ defined therein). So Lorentz forces are always strong, but Epstein drag could dominate over Coulomb drag for $\tilde{U} \lesssim 0.1$. Typical dust-to-gas ratios are also highly uncertain, even with the observational constraints above - in the CGM, metallicities range from $\sim 0.1 \rightarrow 0.3 Z_{\odot}$ around massive galaxies (Leccardi \& Molendi 2008; Tumlinson et al. 2017), but whether the usual dust-to-metals ratio applies is largely unknown.

On top of this, the magnitude of the grain drift is likely to vary tremendously. At one extreme, in the CGM around, say, luminous quasars with $L \sim L_{13} 10^{13} L_{\odot}$, even at a distance $r \sim r_{100} 100 \mathrm{kpc}$ (assuming the QSO is not obscured) we would expect highly supersonic drift with $w_{\mathrm{s}} / c_{\mathrm{s}} \sim 10 r_{100}^{-1}\left(L_{13} / n_{-4} T_{6}\right)^{1 / 2}$ (with $t_{\mathrm{s}} \sim 30 \mathrm{Myr} r_{100} R_{0.1}\left(L_{13} n_{-4}\right)^{-1 / 2}$, and $\left.c_{\mathrm{s}} t_{\mathrm{s}} \sim 3 \mathrm{kpc} r_{100}\left(T_{6} / L_{13} n_{-4}\right)^{1 / 2}\right)$. This is an interesting case in part because it may be the mechanism by which the dust gets into the CGM in the first place (Murray, Quataert \& Thompson 2005; Choi et al. 2012; Hopkins et al. 2016; Ishibashi \& Fabian 2016). At the other extreme, at such large distances from a 'faint' galaxy such as the Milky Way (especially in somewhat denser CGM gas), the radiation pressure on grains is relatively weak and the gravitational forces dominate drift with $w_{\mathrm{s}} / c_{\mathrm{s}} \sim w_{0} / c_{\mathrm{s}} \sim 2\left(M_{\text {halo }} / 10^{12} M_{\odot}\right) R_{0.1} /\left(n_{-4} T_{6} r_{100}^{2}\right)$ (with $t_{\mathrm{s}} \sim 300 \mathrm{Myr} R_{0.1} n_{-4}^{-1} T_{6}^{-1 / 2}$ and $c_{\mathrm{s}} t_{\mathrm{s}} \sim 30 \mathrm{kpc} R_{0.1} n_{-4}^{-1}$ ).

Under the 'fast-drift' (former) conditions, the fast RDI is unstable, but are also in the unusual 'large- $\tau$ ' regime discussed in Section 4.3; the drift is strongly aligned with the magnetic fields and the fastest-growing modes are also aligned. The fastest-growing modes are those analogous to the CR streaming instability, with growth rates at large wavelengths $(\gtrsim \mathrm{kpc}) t_{\text {grow }} \sim \operatorname{Myr}(\lambda / \mathrm{kpc})^{2 / 3} R_{0.1}\left(r_{100} / T_{6}\right)^{1 / 3}\left(L_{13} n_{-4}\right)^{-1 / 6}$ and at short wavelengths $(\lesssim \mathrm{kpc}) t_{\text {grow }} \sim 1000 \mathrm{yr}(\lambda / \mathrm{pc}) R_{0.1} T_{6}^{-1 / 2}$. This can be much faster than characteristic dynamical or free-fall or cooling times ( $\sim \mathrm{Gyr}$ ), sound-crossing times $\left(\sim 10 \mathrm{Myr}(\lambda / \mathrm{kpc}) T_{6}^{-1 / 2}\right)$ or turbulent eddy turnover times $\left(\sim 50 \mathrm{Myr} r_{100}^{1 / 3}(\lambda / \mathrm{kpc})^{2 / 3} T_{6}^{-1 / 2}\right.$, if we assume trans-sonic Kolmogorov turbulence with driving scale $\sim r)$.

Under the 'slow-drift' (latter) conditions the slow Alfvén RDIs will be unstable, but are in the regime where the 'mid- $k$ ' modes are strongly suppressed by high $\tau$. Here 'low- $k$ ' corresponds to very long wavelengths $(\lambda \gtrsim 100 \mathrm{kpc}$ ), so clearly global solutions are needed in this limit. At wavelengths $30 \mathrm{pc} \lesssim \lambda \lesssim 100 \mathrm{kpc}$, the growth rates are suppressed, with $t_{\text {grow }} \gtrsim 3$ Gyr $R_{0.1} r_{100} n_{-4}^{-1 / 2}$, so the instabilities are unlikely to be important. If the RDI can grow at smaller scales (not obvious, owing to dissipative effects), then because $\beta$ is large the gyro resonances can appear (if $w_{\mathrm{s}} \gtrsim v_{\mathrm{A}}$ ) at wavelengths $\lambda \sim 2 \pi w_{\mathrm{s}}\left\langle t_{\mathrm{L}}\right\rangle \sim \operatorname{pc}(\beta / 1000)^{1 / 2} n_{-4}^{-1 / 2} T_{6}^{-1} R_{0.1}^{2}$, with growth times $t_{\text {grow }} \sim$ $3 \times 10^{4} \mathrm{yr}(\beta / 1000)^{1 / 2}(\mu / 0.001)^{-1 / 2} T_{6}^{-3 / 2} n_{-4}^{-1 / 2} R_{0.1}^{2}$.

These instabilities - especially in the 'fast-drift' case - could be critical to understand how dust gets into the CGM in the first place (as needed to explain the observations above; see Ishibashi \& Fabian 2016 and references therein), as well as how dust survives in hot CGM/IGM gas (if, for example, it clumps so as to locally self-shield, or limit/reduce thermal estimates of gas-dust collision rates), where simple sputtering estimates have long suggested it should be rapidly destroyed (Draine \& Salpeter 1979a). Cooling and molecular formation processes in galactic outflows could depend critically on the presence and clumping of dust (e.g. Richings \& Faucher-Giguère 2018). It could again contribute to variations in extinction curves and polarization/scattering (as for the WIM/WNM); these may be especially important for interpretations of scattered and re-emitted light from QSOs at these large distances (e.g. observations of the transverse proximity effect; see Hennawi et al. 2009; Martin et al. 2010). The 'streaming-type' instabilities could allow for behavior analogous to cosmic ray streaming, e.g. transfer of energy from the radiation field (accelerating the dust) to thermal energy of the gas (if the excited Alfvén waves are thermalized through, e.g. turbulence).

\subsubsection{H II regions}

Typical H II regions have $T \sim 10^{4} \mathrm{~K}, n_{\text {gas }} \sim 10^{2} \rightarrow 10^{5} \mathrm{~cm}^{-3}$, modest $\beta \sim 1 \rightarrow 30, f_{\text {ion }} \sim 1, \tilde{U} \sim 1$. If we consider dust at a distance $r=r_{\mathrm{pc}}$ pc from a source (or set of sources) of luminosity $L=L_{6} 10^{6} L \odot$ (e.g. an $\mathrm{OV}$ star), then radiation pressure dominates the acceleration

\footnotetext{
${ }^{26}$ In the CGM around lower-mass galaxies (halo masses $M_{\text {halo }} \ll 10^{12} M_{\odot}$ ), or in the IGM beyond the virial shock, or in cold filaments ('cold flows') accreting onto galaxies, we have $T \sim 10^{4} \mathrm{~K}$, but otherwise similar parameters. Under the 'fast drift' conditions around, e.g. a QSO, the relevant scalings are quite similar to those above (the drift is even more strongly super-sonic, but this does not change the main behavior). The gravity-dominated ('slowdrift') conditions are more interesting: under gravity alone, a simple calculation assuming the gas is hydrostatic would give a drift velocity $w_{\mathrm{s}} / c_{\mathrm{s}} \sim$ $8 r_{100}^{-1}\left(M_{\text {halo }} / 10^{12} M_{\odot}\right)^{1 / 2}\left(R_{0.1} / n_{-4}\right)^{1 / 2}$, which (given lower densities at larger radii) could be super-sonic out to $\gg 10 \mathrm{Mpc}$ scales around $\sim M_{*}$ halos. With high $\tau \sim 10^{3}-10^{5}$, this would excite the fast and Alfvén RDIs and 'streaming-type' mode. However, under these conditions, the gas is not hydrostatic - instead it, and the dust, are likely to be free-falling together onto galaxies or halos. A more detailed estimate of the differential forces is therefore needed to determine the typical drift conditions.
} 
of grains (over, e.g. gravity) with $Q_{\mathrm{abs}} e_{\mathrm{rad}} / e_{\mathrm{thermal}} \sim 8 L_{6} n_{2}^{-1} r_{\mathrm{pc}}^{-2}$ for typical large grains. Because the acceleration falls off with radius (assuming the density falls of less-steeply than $r^{-2}$ ), there is a critical radius at $r_{\mathrm{pc}} \sim 0.5 L_{6}^{1 / 2} n_{2}^{-1 / 2}$, outside of which the drift is sub-sonic, and inside of which it is super-sonic (for more detailed calculations that give similar results, see Draine 2011; Akimkin et al. 2015). Note that the Stromgren radius scales as $r_{\text {stromgren }} \sim 6 \mathrm{pc} L_{6}^{1 / 3} n_{2}^{-2 / 3}$ (assuming the temperature of an OV star) so this is well within the $\mathrm{H}$ II region for all physical densities and luminosities.

Outside $r_{\mathrm{pc}} \gtrsim 0.5 L_{6}^{1 / 2} n_{2}^{-1 / 2}$, the drift is sub-sonic, and Coulomb drag dominates with $w_{\mathrm{s}} / c_{\mathrm{s}} \sim 0.25 L_{6} n_{2}^{-1} r_{\mathrm{pc}}^{-2}$ and $\tau \sim$ $10 R_{0.1}^{-1} n_{2}^{-1 / 2} \beta_{10}^{-1 / 2}$. We find that $t_{\mathrm{s}} \sim 100 \mathrm{yr} n_{2}^{-1} R_{0.1}$ and $c_{\mathrm{s}} t_{\mathrm{s}} \sim 0.001 \mathrm{pc} R_{0.1} n_{2}^{-1}$. For wavelengths $\lambda / \mathrm{pc} \sim(10 \mathrm{au} \rightarrow 0.6 \mathrm{pc}) R_{0.1} / n_{2}$, we are in the regime of the intermediate-wavelength or 'mid- $k$ ' resonance, with the fastest-growing mode either the slow or Alfvén RDI (both scale similarly). This gives growth timescales $t_{\text {grow }} \sim 6000(\lambda / 0.001 \mathrm{pc})^{1 / 2} r_{\mathrm{pc}}^{2} \mathrm{yr} n_{2}^{1 / 4} \beta_{10}^{-1 / 4} L_{6}^{-1}$; comparing to the sound-crossing time at $r$ $\left(t_{\text {sound }}=r / c_{\mathrm{s}} \sim 80000 \mathrm{yr} r_{\mathrm{pc}}\right)$, we see that $t_{\text {grow }} / t_{\mathrm{sound}} \sim 0.1 n_{2}^{1 / 4} L_{6}^{-1} \beta_{10}^{-1 / 4}(\lambda / 0.001 \mathrm{pc})^{1 / 2} r_{\mathrm{pc}}$. Note the expansion timescale of the $\mathrm{H}$ II region is approximately $t_{\text {expand }}=t_{\text {sound }}\left(r=r_{\text {stromgren }}\right) \sim 5 \times 10^{5} \mathrm{yr} L_{6}^{1 / 3} n_{2}^{-2 / 3}$. On the largest spatial scales $\lambda \gtrsim 0.6 \mathrm{pc} R_{0.1} / n_{2}$, the long-wavelength mode dominates, but the growth timescales on these scales are usually longer than the $\mathrm{H}$ II region expansion time. On small scales, from $\lambda \sim 10$ au $R_{0.1} / n_{2}$ down to the dissipation scale $\lambda \sim 0.0006 \mathrm{au} / n_{2}$, we are in the high- $k$ regime. The scales are small enough, and $\tau$ modest, so the high- $k$ Alfvén RDI regime can be reached, with growth times around the viscous scale reaching $t_{\text {grow }} \sim 10$ yr $\beta_{10}^{1 / 6} n_{2}^{-1 / 6} L_{6}^{-2 / 3} R_{0.1} r_{\mathrm{pc}}^{4 / 3}$

Inside the radius $r_{\mathrm{pc}} \lesssim 0.5 L_{6}^{1 / 2} n_{2}^{-1 / 2}$, the drift is super-sonic, and Epstein drag dominates, with $w_{\mathrm{s}} / c_{\mathrm{s}} \sim 4 L_{6}^{1 / 2} n_{2}^{-1 / 2} r_{\mathrm{pc}}^{-1}$ and $\tau \sim 70 r_{\mathrm{pc}} R_{0.1}^{-1} L_{6}^{-1 / 2} \beta_{10}^{-1 / 2}$ (for more detailed calculations that give very similar results; see Akimkin et al. 2015). We find $t_{\mathrm{s}} \sim$ $700 \mathrm{yr} n_{2}^{-1 / 2} L_{6}^{-1 / 2} r_{\mathrm{pc}} R_{0.1}$ and $c_{\mathrm{s}}\left\langle t_{\mathrm{s}}\right\rangle \sim 0.01 \mathrm{pc} r_{\mathrm{pc}} R_{0.1}\left(L_{6} n_{2}\right)^{-1 / 2}$. Under these conditions, a wide range of modes are present. For example, if $r \sim 0.1 \mathrm{pc}, n \sim 10^{4} \mathrm{~cm}^{-3}, \beta \sim 100, L_{6} \sim 1$, then it is possible to be in all three wavelength regimes: (i) at wavelengths $\lambda \gg c_{\mathrm{s}}\left\langle t_{\mathrm{s}}\right\rangle$, the longwavelength/pressure-free mode dominates with $t_{\text {grow }} \sim 300 \mathrm{yr}(\lambda / 0.01 \mathrm{pc})^{2 / 3}\left(r_{\mathrm{pc}} / 0.1\right)^{1 / 3}\left(R_{0.1}^{2} / L_{6} n_{4}\right)^{1 / 6} ;$ (ii) at scales $\lambda \sim\left(10^{-4} \rightarrow 1\right) c_{\mathrm{s}}\left\langle t_{\mathrm{s}}\right\rangle$ the fast MHD-wave RDI is usually fastest-growing and in the mid- $k$ regime, with $t_{\text {grow }} \sim 4 \mathrm{yr}(\lambda / \mathrm{au})^{1 / 2}\left(r_{\mathrm{pc}} / 0.1\right)^{1 / 2}\left(R_{0.1}^{2} / L_{6} n_{4}\right)^{1 / 4}$; and (iii) at still smaller scales down to the dissipation scale $\left(\lambda_{\text {diss }} \sim 10^{8} \mathrm{~cm} n_{4}^{-1}\right)$ we are in the high- $k$ regime with the Alfvén wave-RDI dominant and $t_{\text {grow }} \sim 12$ days $\left(\lambda / \lambda_{\text {diss }}\right)^{1 / 3}\left(r_{\mathrm{pc}} / 0.1\right)^{2 / 3}\left(R_{0.1}^{2} / L_{6} n_{4}^{2}\right)^{1 / 3}$. The gyro-RDI is also present and can produce very fast growth rates at specific wavelengths: e.g. for these parameters, $t_{\text {grow }}^{\text {gyro }} \sim 5 \mathrm{yr}$ around a narrow range of $\lambda \approx 60$ au (a factor of $\sim 6$ faster than the fastest MHD-wave RDI at the same $\lambda$ ). In Fig.7, we show the instability growth timescale for the various RDI families, calculated using parameters relevant for the inner (top panel) and outer (bottom panel) regions of an $\mathrm{H}$ II region.

The RDIs here could be important for a wide range of observed phenomenology. It has long been recognized that dust in $\mathrm{H}$ in regions is critical for their chemistry, depletion of metals (therefore accurate abundance estimates from emission-line estimators), cooling physics, and determining the emergent spectrum (Shields \& Kennicutt 1995). A number of studies have also noted that dust dynamics have a large impact on the expansion of $\mathrm{H}$ II regions via their interaction with gas and radiation pressure, and the drift and dust-gas interaction substantially alters the $\mathrm{H}$ II region expansion rate, densities in the central cavity, dust-to-gas ratios in the interior and swept-up shell, and more (Akimkin et al. 2017). To date, studies of these phenomena have neglected the physics necessary to follow the RDI (3D calculations with magnetized gas, charged dust, drift, Lorentz forces, and back-reaction on gas), but the scalings above suggest that these properties could be radically altered. Moreover, dust clumping induced by the RDI could enhance the leakage of ionizing photons from $\mathrm{H}$ II regions by orders of magnitude (Anderson et al. 2010). The long-wavelength, pressure-free mode will directly drive dust into sharper arcs, shells, and rings concentric around the stars, as observed in many $\mathrm{H}$ in regions. Further, unlike explanations based purely on radiation pressure and shocks, this naturally explains observed shells inside and outside the ionized regions (Topchieva et al. 2017), as well as operating on faster timescales. Meanwhile the fast-wave RDI, on shorter wavelengths, will directly drive dust into filaments or 'whiskers' and dust lanes with extremely sharp fine-structure, as observed in essentially all sufficiently resolved $\mathrm{H}$ il regions (O'Dell et al. 2002; Apai et al. 2005), most famously in $\eta$ Carinae (Morse et al. 1998). These may provide seeds or induce the dust condensations associated with triggered star formation around $\mathrm{H}$ II regions (e.g. Anderson et al. 2012; Deharveng et al. 2015). This may also naturally explain anomalies where the dust knots or filaments do not appear coincident with gas-phase density enhancements on small scales (Garnett \& Dinerstein 2001). The size dependence of the effects could also produce apparent observed grain-size variations across H II regions (Relaño et al. 2016; Hankins et al. 2017).

\subsubsection{SNe ejecta}

In and around SNe explosions, a wide variety of behaviors will manifest at different stages of the event. Immediately after $(t \lesssim 1 \rightarrow 3$ yr) the explosion, dust in the near vicinity of the $\mathrm{SNe}$ (but not within the explosion itself) suddenly sees a very bright source with $L \sim L_{9} 10^{9} \mathrm{M}_{\odot}$. Outside of a sublimation radius $r_{\text {sub }} \sim 0.014 L_{9}^{1 / 2} \mathrm{pc}$, if the ambient density is $n \sim 100 n_{2} \mathrm{~cm}^{-3}$, the nearby dust at radius $r$ from the SNe is radiatively accelerated to extremely high velocities $w_{\mathrm{s}} \sim 4 \times 10^{4} \mathrm{~km} \mathrm{~s}^{-1} n_{2}^{-1 / 2} T_{4}^{1 / 4}\left(r_{\text {sub }} / r\right.$ ) (for more detailed calculations including relativistic effects, see Hoang 2017). (Note that for $r \gg 100 r_{\text {sub }} \sim 1.4 \mathrm{pc}$, the time to accelerate the grains becomes $\gg$ yr, so the maximum velocity drops rapidly). Whether the ambient gas is typical of a cold GMC, or the diffuse WIM, we have $w_{\mathrm{s}} \gg c_{\mathrm{s}}$, with Epstein drag dominating, $\tau \ll 1$ (owing to the very large $w_{\mathrm{s}}$ ), and $\beta \gtrsim 1$. So the rapidly moving dust immediately triggers the fast-wave RDI, which under these conditions is essentially identical to the acoustic RDI from Paper I (with growth timescales $t_{\text {grow }} \sim 3 \mathrm{yr} n_{2}^{-1 / 4} T_{4}^{-1 / 8} R_{0.1}^{1 / 2}\left(r / r_{\text {sub }}\right)^{1 / 2}(\lambda / \mathrm{au})^{1 / 2}$, faster than the expansion time for small scales $\lambda \lesssim$ au).

After several years, the explosion is in the free-expansion phase and dust begins to condense in the ejecta, with what observations indicate has a high, order-unity efficiency (e.g. Bianchi \& Schneider 2007; Matsuura et al. 2015; Owen \& Barlow 2015 ; De Looze et al. 
2017; Temim et al. 2017). Thus, the dust-to-gas ratio inside the ejecta could be quite high, $\mu \gtrsim 0.1$. The ejecta and dust are both freeexpanding to leading order (at a radius $r_{\mathrm{ej}} \sim r_{\mathrm{pc}} \mathrm{pc}$ ), with internal density $n \sim 10\left(M_{\mathrm{ej}} / M_{\odot}\right) r_{\mathrm{pc}}^{-3}$ and velocity $v \sim v_{4} 10^{4} \mathrm{~km} \mathrm{~s}{ }^{-1}$, adiabatically cooled to $T \sim 20 \rightarrow 100 \mathrm{~K}$, and likely with large magnetic fields $\beta \sim 10^{-6}\left(M_{\mathrm{ej}} / M_{\odot}\right) r_{\mathrm{pc}}^{-3} T_{2} B_{0 \text {, mG }}^{-2}$ (Reynolds, Gaensler \& Bocchino 2012), suggesting large $\tau \sim 2 \times 10^{4} r_{\mathrm{pc}}^{3} T_{2}^{1 / 2} R_{0.1}^{-1}\left(M_{\mathrm{ej}} / M_{\odot}\right)^{-1} B_{0, \mathrm{mG}}$ (where $B_{0, \mathrm{mG}} \equiv\left|\mathbf{B}_{0}\right| / \mathrm{mG}$ ). Radiative acceleration of the dust is unimportant after several years; however, the gas ejecta may be accelerating (if being driven by relativistic winds in e.g. a pulsar wind nebula) or decelerating (from sweeping up gas in the ISM). Consider (for simplicity) the latter: if the ambient density is $n \sim 1 \mathrm{~cm}^{-3}$, then momentum (or energy) conservation demands that the gas has decelerated by a factor $\sim 1-M_{\mathrm{swept}} / M_{\mathrm{ej}} \sim 1-0.1 n_{0} r_{\mathrm{pc}}^{3}\left(M_{\mathrm{ej}} / M_{\odot}\right)^{-1}$, which (if the gas were still cold) would imply a highly super-sonic drift velocity $w_{\mathrm{s}} \sim 1000 \mathrm{~km} \mathrm{~s}^{-1} v_{4} \cdot{ }^{27}$ However, the gas that is de-celerated is being processed through a reverse shock as well, and the (Epstein) stopping time in this phase is itself comparable to the expansion time $\left(\sim r_{\mathrm{ej}} / v_{\mathrm{eg}}\right)$, $t_{s} / t_{\text {expand }} \sim\left(R_{0.1} / n_{0} r_{\mathrm{pc}}\right)^{1 / 2}$, so it is unclear what the drift velocity in the cold portion of the ejecta will actually be without a global, timedependent solution. The drift is therefore likely trans-Alfvénic and either trans-sonic or highly super-sonic, depending on whether the dust resides in the reverse-shocked region or remains in 'cold' regions (or drifts 'out of' the reverse-shocked region on a timescale $\lesssim t_{\mathrm{s}}$ ). If the 'cold' case can be realized, strong gyro-resonances are present with growth times $t_{\text {grow }} \sim 40 \mathrm{yr} R_{0.1}^{2} B_{0 \text {, mG }}^{-1} T_{2}^{-1}(\mu / 0.1)^{-1 / 2}$ at relatively long wavelengths $\lambda \sim(0.01 \rightarrow 0.04)$ pc $R_{0.1} r_{\mathrm{pc}}^{3} T_{2}^{1 / 2}\left(M_{\mathrm{ej}} / M_{\odot}\right)^{-1}\left(w_{\mathrm{s}} / 1000 \mathrm{~km} \mathrm{~s}^{-1}\right)^{-1}$, while the Alfvén RDI dominates at shorter wavelengths with $t_{\text {grow }} \sim 3 \mathrm{yr} B_{0, \mathrm{mG}}^{-1 / 2} r_{\mathrm{pc}}^{3 / 2} R_{0.1}^{1 / 2}(\lambda / \mathrm{au})^{1 / 2}\left(M_{\mathrm{ej}} / M_{\odot}\right)^{-1 / 2}\left(w_{s} / 1000 \mathrm{~km} \mathrm{~s}^{-1}\right)^{-1 / 2}$. The 'hot' case will essentially be the next (Sedov-Taylor) phase.

Once the reverse shock has propagated through the ejecta (after it sweeps up a mass comparable to its own), the SNe remnant enters the energy-conserving Sedov phase, with shell densities $n \sim 4 n_{\mathrm{ISM}} \sim n_{0} \mathrm{~cm}^{-3}$, velocities $v_{\mathrm{ej}} \sim 840 \mathrm{~km} \mathrm{~s}^{-1}\left(E_{51} / n_{0} r_{10}^{3}\right)^{1 / 2}$, and postshock temperatures $T \sim 1.6 \times 10^{7} E_{51} n_{0}^{-1} r_{10}^{-3}$ (where $E_{51}$ is the ejecta energy in $10^{51} \mathrm{erg}$ and the ejecta are at radius $r_{10} 10 \mathrm{pc}$ ). The gas in the ejecta is de-celerating according to energy conservation; adopting the usual Sedov solution gives a drift velocity $w_{\mathrm{s}} / c_{\mathrm{s}} \sim$ $0.8 R_{0.1} n_{0}^{-1} r_{10}^{-1}(\tilde{U} / 0.3)^{-2}$. So we expect trans- or sub-sonic drift with Coulomb drag dominating (although see the caveats about $\tilde{U}$ at these temperatures in the CGM discussion above, which could lead to Epstein drag dominating), with $c_{\mathrm{s}} t_{\mathrm{s}} \sim(1 \mathrm{pc}) R_{0.1} n_{0}^{-1}(\tilde{U} / 0.3)^{-2}$, and $t_{\mathrm{s}} \sim 3000 \mathrm{yr} R_{0.1} n_{0}^{-1 / 2} r_{10}^{3 / 2} E_{51}^{-1 / 2}(\tilde{U} / 0.3)^{-2}$. We expect modest $\beta \sim 1$, giving very large $\tau \sim 10^{6} E_{51} n_{0}^{-3 / 2} \beta^{-1 / 2} R_{0.1}^{-1} r_{10}^{-3}(\tilde{U} / 0.3)^{-1}$. Importantly, under these conditions it is generally believed that a large fraction of the dust is efficiently destroyed via sputtering, so $\mu$ may be reduced to $\mu \ll 0.001$. Given the low $\mu$, subsonic or transsonic drift, and very large $\tau$ (which suppresses the mid- $k$ slow modes), the RDIs here may be heavily suppressed during these stages (with growth times $\gtrsim$ Myr), except at very small scales $\left(t_{\text {grow }} / t_{\text {expansion }} \sim\right.$ $E_{51}^{1 / 2}(\mu / 0.001)^{-1 / 2} r_{10}^{-5 / 2} n_{0}^{-5 / 4} \beta^{-1 / 4}\left(\lambda / 10^{10} \mathrm{~cm}\right)^{1 / 2}$ for $\lambda \ll$ au $)$.

After the remnant sweeps up a mass $\sim 3000 M_{\odot}$, it enters the momentum-conserving/cooling/snowplow phase. The shell continues to decelerate (conserving momentum), with velocity $v_{\mathrm{ej}} \sim 170 \mathrm{~km} \mathrm{~s}^{-1} n_{0}^{-1} r_{30}^{-3} \tilde{p}$ (where $\tilde{p}$ is the 'terminal momentum' at the cooling radius, relative to the value typically measured in simulations of $\sim 4 \times 10^{5} M_{\odot} \mathrm{km} \mathrm{s}^{-1}$; Cioffi, McKee \& Bertschinger 1988; Thornton et al. 1998; Kim \& Ostriker 2015; Martizzi, Faucher-Giguère \& Quataert 2015; Walch \& Naab 2015; Hopkins et al. 2017). The shell is at $r_{30} 30$ pc, but it is has cooled $\left(T \sim 10^{2} \rightarrow 10^{4} \mathrm{~K}\right)$, and is more dense at the front owing to the radiative shock $\left(n_{\text {shell }} \sim \mathcal{M}^{2} n_{\text {ISM }}\right.$; although since the shell is magnetized the jump may be weaker, so we will simply write $\left.x_{\text {jump }}=n_{\text {shell }} / n_{\mathrm{ISM}}\right)$. This sources a drift $w_{\mathrm{s}} / c_{\mathrm{s}} \sim 5 \tilde{p} R_{0.1}^{1 / 2} n_{0}^{-3 / 2} r_{30}^{-7 / 2} T_{4}^{-1 / 2} x_{\text {jump }}^{-1 / 2}$, with $t_{\mathrm{s}} / t_{\text {expansion }} \sim 0.3\left(R_{0.1} / n_{0} r_{30} x_{\text {jump }}\right)^{1 / 2}$ and $c_{\mathrm{s}} t_{\mathrm{s}} \sim \operatorname{pc}\left(n_{0} R_{0.1} T_{4} / x_{\text {jump }}\right)^{1 / 2} \tilde{p}^{-1} r_{30}^{7 / 2}$. Thus, over the range of temperatures and radii (or entrained masses) over which this phase is relevant, we should expect a broad range from sub-to-supersonic drift, with $\beta \sim 1$, Epstein drag dominating (since the gas is cold), and large $\tau \sim 2000 n_{0} \tilde{p}^{-1} T_{4}^{3 / 2} \beta^{-1 / 2} R_{0.1}^{-3 / 2} r_{30}^{7 / 2}$. Since the gas and dust mass is primarily that which is entrained, and sputtering is no longer efficient, the dust-to-gas ratio should reflect the ISM $(\mu \sim 0.01)$. Under these conditions, a wide range of modes is present: at wavelengths close to $\lambda \sim 2 \pi\left\langle t_{\mathrm{L}}\right\rangle w_{\mathrm{s}} \sim 0.01 \mathrm{pc} \tilde{p} x_{\text {jump }}^{-1} n_{0}^{-2} R_{0.1}^{5 / 2} \beta^{1 / 2} T_{4}^{-3 / 2} r_{30}^{-7 / 2}$ (as well as around $\lambda \sim 2 \pi\left\langle t_{L}\right\rangle v_{f, 0}$ ), the gyro modes have very rapid growth timescales $t_{\text {grow }} \sim \mu^{-1 / 2}\left\langle t_{L}\right\rangle \sim 300$ yr $R_{0.1}^{2}\left(\beta / n_{0} x_{\text {jump }}\right)^{1 / 2} T_{4}^{-3 / 2}$ (compare to the expansion time, $\left.t_{\text {expansion }} \sim 1.7 \times 10^{5} \mathrm{yr} n_{0} \tilde{p}^{-1} r_{30}^{4}\right)$. At shorter wavelengths the mid- $k$ regime of the high- $\tau$ Alfvén/fast-wave RDI dominates with $t_{\text {grow }} / t_{\text {expansion }} \sim(\lambda / 0.001 \mathrm{pc})^{1 / 2} r_{30}^{3} n_{0}^{-5 / 4}\left(x_{\text {jump }} / \beta\right)^{1 / 4}\left(T_{4} / \tilde{p} R_{0.1}\right)$, until at very short wavelengths $\left(\lambda \ll r_{30}^{7 / 2}\right.$ au $)$ where the high- $k$, high$\tau$-enhanced Alfvén RDI dominates with $t_{\text {grow }} / t_{\text {expansion }} \sim 0.001(\lambda / \mathrm{au})^{1 / 3} \tilde{p}^{1 / 3} r_{30}^{-5 / 3} R_{0.1}^{2 / 3}\left(n_{0} T_{4}\right)^{1 / 2}\left(x_{\text {jump }} / \beta\right)^{1 / 6}$.

This will have a wide range of consequences. The initially accelerated dust in the vicinity of the SNe will clump strongly, potentially shielding it against sputtering, and changing the cooling, abundance, and molecular properties of the ISM through which it moves (see the discussion of the instabilities in the warm and cold ISM). During the early free expansion phase, as many detailed theoretical studies have pointed out, clumpiness in the ejecta, and especially of the initial grain 'seeds,' can radically alter the efficiency of grain growth, and therefore the ensuing grain size distribution, composition, and survival through the energy-conserving phase in SNe ejecta (Bianchi \& Schneider 2007; Silvia, Smith \& Shull 2010; Hirashita \& Kuo 2011; Sarangi \& Cherchneff 2015). This may, in part, be related to the very high condensation efficiencies observed in some SNe referenced above. Sufficiently non-linear manifestations of the highly supersonic RDIs can lead to dust 'filaments', which can effectively drift 'through' the shock (Moseley et al., in preparation), potentially providing a means for some dust to avoid the reverse shock entirely. The strong gyro-RDI modes may also play an important role in amplifying or altering the structure of magnetic fields in SNe remnants. In both this energy-conserving stage and later (snowplow) stages, the Alfvén RDI will generate dust-to-gas

${ }^{27}$ More accurately calculating the equilibrium drift velocity for a shell expanding into a uniform ambient medium, we obtain $w_{\mathrm{s}} \approx$ $2000 \mathrm{~km} \mathrm{~s}^{-1} v_{4}\left(n_{0} R_{0.1}\right)^{1 / 2} r_{\mathrm{pc}}^{5 / 2}\left(M_{\mathrm{ej}} / M_{\odot}\right)^{-1}$ while $w_{\mathrm{s}} \ll v_{\mathrm{ej}}$. 
fluctuations on small scales, which could source turbulence in the shell and the ubiquitously observed dust 'streamers' and micro-structure. This could, in turn, significantly alter interpretations of the far-IR and sub-mm fluxes of the remnants.

\subsubsection{Coronal dust}

Observations have long indicated the presence of dust throughout the solar corona (the 'F-corona') at least down to radii $r<2 R_{\odot}$, with the density of grains rising proportionally to $\sim r^{-1}$, similar to gas (see e.g. Mann et al. 2004, 2006, for reviews). This includes a range of sizes and compositions (with larger grains $R_{0.1} \gg 1$ more easily observed, but smaller grains likely to appear closer to the Sun, especially if deposited by sub-grazing comets; e.g. Mukai et al. 1974; Kimura \& Mann 1998). More recently similar coronae have been inferred around a large number of nearby stars (Kral et al. 2017).

In the solar corona, some typical parameters are $T \sim 10^{6} \mathrm{~K}, n \sim 10^{8} \mathrm{~cm}^{-3}, \beta \sim 0.001 \beta_{-3}$ (scaling with distance from the Sun). If dust is at a distance $r_{\odot} \equiv r / R_{\odot}$ from the Sun or another star with luminosity $L_{0}=L / L_{\odot}$, then radiative acceleration produces a drift velocity ${ }^{28}$ (before projection onto the field) of $w_{0} / c_{\mathrm{s}} \sim 30\left(L_{0} Q_{\mathrm{abs}} / r_{\odot} n_{8} T_{6}\right)^{1 / 2}, t_{\mathrm{s}} \sim 260 \sec R_{0.1}\left(r_{\odot} / L_{0} Q_{\mathrm{abs}} n_{8}\right)^{1 / 2}, c_{\mathrm{s}} t_{\mathrm{s}} \sim 0.04 R_{\odot} R_{0.1}\left(T_{6} / L_{0} Q_{\mathrm{abs}} n_{8}\right)^{1 / 2}$, and $\tau \sim 100 T_{6}^{3 / 2} \tilde{U} R_{0.1}^{-1}\left(r_{\odot} / L_{0} Q_{\text {abs }} \beta_{-3}\right)^{1 / 2}$. Thus, coronal dust is in a unique regime in several respects: $\beta \ll 1$ and the dust is drifting super-sonically (in the Epstein regime), but sub-Alfvénically, and the system has moderately large $\tau$.

If we evaluate the growth rates using parameters appropriate for grains around the base of the corona $\left(Q_{\mathrm{abs}} \sim 0.1, \tilde{U} \sim 0.3\right.$ per the CGM discussion above, at $T_{6}=\beta_{-3}=n_{8}=L_{0}=r_{\odot}=1$ ), then rather remarkably, the parallel, acoustic quasi-drift mode has a growth time $t_{\text {grow }} \sim 1.2 R_{0.1}(0.01 / \mu)$ days over a wide range of $\lambda \gg R_{\odot}$ (formally up to $\sim$ au, but clearly a global solution is needed on such large scales). At shorter (intermediate) wavelengths the fastest growing modes are the Alfvén-wave RDIs in the mid- $k$ regime with growth timescales $t_{\text {grow }} \sim 1.7$ day $\left(\lambda / R_{\odot}\right)^{1 / 2}(0.01 / \mu)^{1 / 2}$ (this estimate is only weakly dependent on grain size), while at very short wavelengths $\lambda \lesssim 10 \mathrm{~km}$, the high- $k$ Alfvén RDI appears with $t_{\text {grow }} \sim \sec (\lambda / \mathrm{km})^{1 / 3}(0.01 / \mu)^{1 / 3}$. Note that we have kept the the dust-to-gas ratio $\mu$ arbitrary in these estimates, as this is highly uncertain in the near vicinity of the Sun (extrapolating the models reviewed in Mann et al. 2004 suggests $\mu \sim 10^{-4}$ $\rightarrow 10^{-3}$ at $\lesssim 4 R_{\odot}$, but the value is expected to drop rapidly as $r \rightarrow R_{\odot}$ owing to a number of effects).

It has long been recognized that complicated dust dynamics (formation, destruction, drift owing to radiation, gravity, and the PoyntingRobertson effect) and dust-gas interactions can produce features and enhancements in the F-corona and 'rings' of dust observed at times (Mukai \& Yamamoto 1979; Kimura, Mann \& Mukai 1998; Mann, Krivov \& Kimura 2000; Kobayashi et al. 2009). However, these studies have not included the physics necessary to follow the RDI, which could lead to much larger local enhancements (on long wavelengths, the parallel modes tend to induce ring/shell/arc structures). This could be important for dust-ring dynamics, and perhaps related to historical claims of variability (e.g. Prasad 1995; Ohgaito et al. 2002). The Alfvén RDI (at shorter wavelengths) directly sources strong interactions between the dust and the magnetic fields, so may be important to understand the observed interactions of dust with the particles and magnetic fields of coronal mass ejections and the solar wind (Ragot \& Kahler 2003). It is likely necessary to account for the RDI in understanding the observed fluctuations and structure functions in observed dust properties carried by the solar wind (Strub, Krüger \& Sterken 2015). Additionally, more recent observations have argued for time-variable streamers and other small-scale structures in the F-corona, which could be driven by the RDI (Shopov et al. 2008).

\subsubsection{Winds around cool stars}

Around cool, giant stars, dust forms in the photosphere and may be critical for launching winds. These conditions are high-density $(n \gtrsim$ $10^{12} \mathrm{~cm}^{-3}$ ), low-temperature $\left(T \lesssim 1000 \mathrm{~K}\right.$ ) and modestly magnetized (intermediate $\beta$ ), with $R_{d} \sim 0.001 \rightarrow 1 \mu$ m, suggesting $\tau \ll 1$ with Epstein drag dominating over Coulomb drag. Both simple analytic arguments ${ }^{29}$ and detailed simulations (MacGregor \& Stencel 1992; Hartquist \& Havnes 1994; Woitke 2006) have shown that the drift velocities are transsonic, with $w_{\mathrm{s}} \sim 0.1 \rightarrow 10 c_{\mathrm{s}}$, depending on local conditions (e.g. different stars or different regions within the same wind).

Under these conditions, the regions with super-sonic drift will be dominated by the fast-magnetosonic mode resonance, which for low- $\tau$ and $w_{\mathrm{s}} \gg v_{\mathrm{f}, 0}$ behaves effectively identically to the acoustic RDI studied in detail in Paper II. Even if $\beta \ll 1$, the only difference in this regime from our Paper II scalings is the replacement $c_{\mathrm{s}} \rightarrow v_{\mathrm{f}, 0}$. Under these conditions we expect the acoustic or fast-magnetosonic RDI to be important, with growth timescales much faster than wind expansion times even for the largest scale modes (wavelengths of order the distance from the star), reaching growth timescales as fast as $t_{\text {grow }} \ll 1 \mathrm{~s}$ on the smallest (viscous) scales $(\lambda \ll 1 \mathrm{~m})$. Essentially all scales

\footnotetext{
${ }^{28}$ For larger $(\gg 1 \mu \mathrm{m})$ grains around a solar-type star, gravity dominates over radiation pressure, so we can consider this as the dominant source of drift, but the qualitative scalings above are identical. The Poynting-Robertson effect adds another external acceleration to source drift, but this is sub-dominant by a factor $\sim w_{\mathrm{s}} / c$. Even in a gravity-dominated circular orbit, gas pressure means the gas rotates at sub-Keplerian speeds, generating a relative dust-gas drift $w_{\mathrm{s}} / c_{\mathrm{s}} \sim$ $c_{\mathrm{S}} / V_{c}$ (the drift which matters here), which usually dominates over Poynting-Robertson.

${ }^{29}$ To summarize from Paper II: in a wind with $\rho=\dot{M} /\left(4 \pi r^{2} v_{\text {wind }}\right)$, with $v_{\text {wind }}=v_{10} 10 \mathrm{~km} \mathrm{~s}^{-1}, r=r_{100} 100 R_{\odot}, \dot{M} \sim \dot{M}_{-3} 10^{-3} \mathrm{M}_{\odot} \mathrm{yr}^{-1}$, and $T \sim$ $1000 \mathrm{~K}$, around a giant with luminosity $L \sim L_{5} 10^{5} L_{\odot}$, assuming geometric absorption gives $w_{\mathrm{s}} / c_{\mathrm{S}} \sim 2\left(L_{5} v_{10} / \dot{M}_{-3} T_{3}\right)^{1 / 2}$ (with corresponding $t_{s} \sim$ $1 \sec R_{0.1} r_{100}^{2}\left(v_{10} / L_{5} \dot{M}_{-3}\right)^{1 / 2}$ and $\left.c_{s} t_{s} \sim 3 \times 10^{5} \mathrm{~cm} T_{3}^{1 / 2}\left(t_{s} / \mathrm{sec}\right)\right)$. The long-wavelength $\left(\lambda \gtrsim 10^{8} \mathrm{~cm}\right)$, mid- $k$, and short-wavelength (from $10^{4} \mathrm{~cm}$ to the viscous scale $\sim 10 \mathrm{~cm})$ regimes at are all present and relevant, with growth rates at long wavelengths of $t_{\text {grow }} / t_{\text {wind }} \sim 0.02 v_{10}^{4 / 3}\left(R_{0.1} r_{100} / \dot{M}_{-3}\right)^{1 / 3} T_{3}^{-1 / 2}(\lambda / r)^{2 / 3}$ (where $t_{\text {wind }}=r / v_{\text {wind }}$ is the wind expansion time) and at short wavelengths (approaching the viscous scale) of $t_{\text {grow }} \sim 0.1 \mathrm{sec} R_{0.1}^{2 / 3} T_{3}^{-1 / 2}$
} 
are unstable, and the interesting modes span the long-wavelength, mid- $k$ and high- $k$ resonant regimes. For more detailed discussion of these cases, we refer to Paper II.

However, the addition of the slow-mode resonance, when we consider MHD, is of particular interest for the sub-sonic drift regime. Recall, for modest $\beta$ and low $\tau$, the slow-mode resonant RDI has growth rates that scale similarly to the fast-mode RDI, except for being suppressed by a factor $\sim\left(w_{\mathrm{s}} / v_{\mathrm{f}, 0}\right)^{\alpha}$ with $\alpha$ in the range $\sim 2 / 3 \rightarrow 1$. But since the drifts expected in cool-star winds are only modestly sub-sonic, this is not a large suppression factor (this also likely means that they will not be strongly suppressed by ambipolar diffusion, if present). This situation is quite different from the neutral hydrodynamic system (acoustic instability; Paper II), where there is a very sharp change in the character of the instabilities for $w_{\mathrm{s}}<c_{\mathrm{s}}$ (at which point the resonances cease to exist entirely in the absence of magnetic fields). So we expect a rather smooth transition between the two cases: the behavior for sub-sonic or super-sonic drift will be similar, but with faster growth rates in regions of faster drift. In these systems the RDI can source both large-scale features commonly seen in the outflows (arcs/shells/rings, and global dust asymmetries; see e.g. Morris 1993; Winters, Dominik \& Sedlmayr 1994; Deguchi 1997; Balick \& Frank 2002), as well as the ubiquitous observed dust knots/filaments/fliers/streamers (O'dell \& Handron 1996; Balick et al. 1998; O'Dell et al. 2002; Matsuura et al. 2009). In addition, this provides a natural mechanism to generate observed gas clumpiness and turbulence in outflows (Fong, Meixner \& Shah 2003; Young et al. 2003; Ziurys et al. 2007; Agúndez, Cernicharo \& Guélin 2010; Cox et al. 2012).

\subsubsection{The neutral and cold interstellar medium (GMCs, star-forming regions, and AGN torii)}

In the cold $(T \ll 1000 \mathrm{~K})$ ISM (e.g. the cold-neutral and molecular media, including molecular clouds, star-forming regions, galactic nuclei, the dusty obscuring 'torii' and narrow-line regions around AGN), we have $T \sim 10 \rightarrow 1000 \mathrm{~K}, R_{d} \sim 0.01 \rightarrow 10 \mu \mathrm{m}, n_{\text {gas }} \sim 10 \rightarrow 10^{6} \mathrm{~cm}^{-3}$, $\beta \sim 1$, and $f_{\text {ion }} \sim 10^{-8} \rightarrow 10^{-5}$. Under these conditions, Epstein drag dominates over Coulomb drag, Lorentz forces are weak $(\tau \ll 1)$, and the drift velocities tend to vary from trans-sonic for the smallest grains, through to highly super-sonic for large grains (especially around bright sources like massive stars or star clusters or AGN, where radiation pressure can be sufficient to launch strong outflows of gas and dust). For example, one finds $w_{\mathrm{s}} \gtrsim 100 c_{\mathrm{s}} \gg v_{\mathrm{f}, 0}$ in quasar-driven outflows in the torus or narrow-line region.

Given the high drift velocities, we generally expect the fast-magnetosonic RDI with $\tau \ll 1$ to dominate; as noted above, this is essentially identical to the acoustic RDI from Paper II. As shown there, the growth timescale can be many orders of magnitude faster than competing dynamical timescales in starburst and AGN nuclei, and the most interesting modes are usually the long-wavelength and mid- $k$ resonant modes. Since the scalings are essentially unchanged, we refer interested readers to Paper II for detailed discussion of these cases. ${ }^{30}$

As discussed in Paper II, the RDI could be an important part of observed phenomena ranging from the well-studied clumpiness, substructure, and turbulence in the dusty AGN 'torus' (see e.g. Krolik \& Begelman 1988; Nenkova et al. 2008; Mor, Netzer \& Elitzur 2009; Hönig \& Kishimoto 2010, and references therein), time variability in AGN dust obscuration (McKernan \& Yaqoob 1998; Risaliti, Elvis \& Nicastro 2002), AGN winds driven by radiation pressure on dust (Murray et al. 2005; Elitzur \& Shlosman 2006; Miller, Turner \& Reeves 2008; Wada, Papadopoulos \& Spaans 2009; Roth et al. 2012), observed dust-gas segregation in GMCs (Padoan et al. 2006) and abundance anomalies sourced by these (Hopkins 2014; Hopkins \& Conroy 2017), dust growth and coagulation (believed to occur primarily in the cold, dense ISM; Draine 2003 and references therein), dust chemistry/cooling physics critical for star formation and formation of complex organic compounds and molecules (Goldsmith \& Langer 1978; Dopcke et al. 2013; Chiaki et al. 2014; Ji, Frebel \& Bromm 2014), radiation-pressure-driven outflows from massive stars (Murray et al. 2005; Thompson, Quataert \& Murray 2005; Krumholz, Klein \& McKee 2007; Hopkins, Quataert \& Murray 2011; Grudić et al. 2016), and thermal regulation of proto-star formation via heating dust in coupled dust-gas cores (Guszejnov, Krumholz \& Hopkins 2016).

Of course, the drift velocities will be sub-sonic for sufficiently small grains, and even for larger grains in certain regions (given the highly inhomogenous nature of these systems). In this case, as noted in the discussion of cool stars above, the slow-magnetosonic RDI does become interesting, and (since the drift is only modestly sub-sonic) produces instabilities with similar behavior and growth rates to the fast-mode RDI. However as noted in Section 8, ambipolar diffusion will suppress the slow resonance when $w_{\mathrm{s}} \ll c_{\mathrm{s}}$ and $f_{\text {ion }} \ll 10^{-6}$ (it has little or no effect on the fast resonances discussed above).

\subsubsection{Proto-planetary discs, proto-stellar discs, and planetary atmospheres}

In proto-planetary or proto-stellar discs, the high densities and very low ionized fractions mean that Epstein (or Stokes, for large $\gtrsim \mathrm{cm}$-sized pebbles) drag strongly dominates over Coulomb drag. Similarly Lorentz forces are extremely weak $\left(\tau \ll 10^{-10}\right)$, and magnetic fields (even in the active regions of the disc) are likely relatively weak also $(\beta \gg 1)$. Drift velocities are highly sub-sonic $w_{\mathrm{s}} \ll 10^{-2} c_{\mathrm{s}}(\mathrm{Chiang} \&$ Youdin 2010), so the acoustic RDI from Paper II has very low maximum growth rates and is unlikely to be important. In principle, the slow-mode

\footnotetext{
${ }^{30}$ To summarize the scalings in Paper II: for an AGN with luminosity $L \sim L_{13} 10^{13} L \odot$, and dusty torus with inner radius around the dust sublimation radius $\sim 0.6 \mathrm{pc} L_{13}^{1 / 2}$, midplane column density $N_{26} 10^{26} \mathrm{~cm}^{-2}$, temperature $T \sim 1000 \mathrm{~K}$, the (Epstein) stopping time is $t_{\mathrm{s}} \sim 12 \mathrm{hr} R_{0.1} L_{13}^{1 / 4} N_{26}^{-1 / 2}, c_{\mathrm{s}} t_{\mathrm{s}} \sim$ $10^{10} \mathrm{~cm} R_{0.1} L_{13}^{1 / 4}\left(T_{3} / N_{26}\right)^{1 / 2}, w_{\mathrm{s}} / c_{\mathrm{s}} \sim 100 L_{13}^{1 / 4} N_{26}^{-1 / 2}$, and $t_{\text {grow }} \sim 10 \mathrm{yr} R_{0.1}^{1 / 3} L_{13}^{-1 / 12} N_{26}^{1 / 6}\left(Z / Z_{\odot}\right)^{-1 / 3}(\lambda / 0.1 \mathrm{pc})^{2 / 3}$ down to $\lambda \lesssim$ au, with $t_{\text {grow }} \sim t_{\mathrm{s}} \sim 10$ $\mathrm{h}$ for modes approaching the viscous scale. For a $\operatorname{GMC}\left(T \sim 10-100 \mathrm{~K}, n \sim 1-10^{3} \mathrm{~cm}^{-3}\right)$ which has converted a fraction $\sim 0.1 \epsilon_{0.1}$ of its mass into stars (assuming a standard stellar initial mass function and that none have exploded, to obtain $e_{\text {rad }}$ ), and total size $r \sim r_{10} 10$ pc, we estimate $w_{\mathrm{s}} \sim 10 r_{10}^{1 / 2} \epsilon_{0.1}^{1 / 2}$, $t_{\mathrm{s}} \sim 4 \times 10^{4} \mathrm{yr} R_{0.1} n_{2}^{-1}\left(r_{10} \epsilon_{0.1}\right)^{-1 / 2}, c_{\mathrm{s}} t_{\mathrm{s}} \sim 0.04 \mathrm{pc} R_{0.1} n_{2}^{-1}\left(T_{2} / r_{10} \epsilon_{0.1}\right)^{1 / 2}$, giving $t_{\text {grow }} \sim 0.3 \mathrm{Myr}(\lambda / 0.1 \mathrm{pc})^{1 / 2}\left(R_{0.1} / n_{2}\right)^{1 / 2}\left(r_{10} T_{2} \epsilon_{0.1}\right)^{1 / 4}$.
} 
RDI, even with $\beta \gg 1$, has growth rates that become arbitrarily large at high- $k$. However, as discussed in detail in Squire \& Hopkins (2017a), the ionization fractions in proto-planetary discs are sufficiently low that non-ideal magnetic dissipation terms (e.g. Ohmic resistivity and ambipolar diffusion) cannot be neglected. As discussed above (Section 8), these terms suppress the short-wavelength slow-mode growth. Instead, Squire \& Hopkins (2017a) focus on several other classes of RDI that could be important for planetesimal growth, including the well-studied 'streaming instability' (Youdin \& Goodman 2005), which is an RDI with disc epicyclic oscillations, the related vertical-epicyclic RDI or 'settling instability' (which has growth times comparable to the disc dynamical time at all grain sizes), and the Brunt-Väisälä RDI.

The situation is similar in most planetary atmospheres. There, the comparatively weak centrifugal/coriolis forces suggest that the Brunt-Väisälä RDI is likely to be more important than RDIs associated with epicyclic oscillations.

\section{DISCUSSION}

We have studied the linear stability of dust-gas systems where the gas obeys the ideal MHD equations and dust experiences some combination of an arbitrary drag law and Lorentz forces. We show that such systems exhibit a broad spectrum of 'RDIs', as generically predicted in Paper I, with several unstable modes at all wavenumbers. We identify a large number of different instability families, each of which has distinct behavior. These are summarized in Section 2, but include MHD-wave RDI families (the 'Alfvén RDI,' 'Fast-Magnetosonic RDI,' and 'Slow-Magnetosonic RDI'), the 'gyro-resonant RDI' families (one familiy for each MHD wave family: Alfvén-gyro RDI, fast-gyro RDI, and slow-gyro RDI), the 'pressure free' mode (at sufficiently long wavelengths), the aligned acoustic modes (out-of-resonance modes), and the 'cosmic-ray streaming' modes. Each of these families has distinct behaviors, structure, and possible resonances, and can be the fastest growing instability over some range of wavelengths under different conditions.

Although some of these instabilities are, in a general sense, related to certain well-known instabilities (e.g., of cosmic rays), none of them has (to our knowledge) been previously recognized before Paper I. Although we first identified the existence of the MHD-wave RDI families in Paper I, several features of this work are novel compared to previous works, in particular the inclusion of grain charge (grain Lorentz forces). This significantly modifies the phenomenology of the instabilities, increasing the importance of the Alfvén-wave-related modes, and destabilizing the gyro-resonant families and the 'cosmic ray streaming' mode (which depend on Lorentz forces on dust).

In a very broad sense, the qualitative behavior of the pressure-free mode and MHD-wave RDI families is similar to that of the 'acoustic RDI' studied in Paper II. At sufficiently long wavelengths, the pressure-free mode has the fastest growth rates (and is nearly identical in non-magnetized fluids). At intermediate and short wavelengths, the fastest-growing MHD-RDI modes are usually those with wavevector $\mathbf{k}$ oriented at a 'resonant angle,' such that $\mathbf{w}_{\mathrm{s}} \cdot \mathbf{k}=v_{\mathrm{p}}(\hat{\mathbf{k}}) k$, where $v_{\mathrm{p}}(\hat{\mathbf{k}})$ is the phase speed of the corresponding MHD wave at that angle. The growth rates increase without limit towards short wavelengths, and are only weakly dependent on the dust-to-gas mass ratio $\mu$ (as $\sim \mu^{1 / 3}$ or $\sim \mu^{1 / 2}$ ), the strength $(\beta)$ and orientation $\left(\hat{\mathbf{B}}_{0}\right)$ of magnetic fields, the details of the dust drag law (e.g. Epstein, Stokes, Coulomb, or other drag laws, which we parametrize through $\zeta_{s}, \zeta_{w}$, etc.), and the dust charge (including any dependence on temperature, parametrized through $\zeta_{q}$ ). We show that Lorentz forces on charged grains do not generally suppress these instabilities; in fact Lorentz forces can enhance the growth rates in some regimes. The most important difference between the MHD-wave RDI cases studied here and the acoustic RDI (Paper II) is the existence of the slow and Alfvén waves. Because these have phase velocities that can be arbitrarily small, there is always a range of angles that satisfy the resonance condition and produce rapid growth rates, at any non-zero drift velocity. In contrast, in the un-magnetized acoustic case, the drift must be super-sonic $\left(w_{\mathrm{s}} \geq c_{\mathrm{s}}\right)$ in order to satisfy the resonance condition.

All of the MHD-wave RDI families generate compressive modes in the dust that directly source exponentially growing dust-to-gas fluctuations. For the magnetosonic (fast and slow) RDIs, this occurs via the interaction of dust drag with the acoustic gas density fluctuations. For the Alfvén RDI the perturbed gas mode is, to leading order, incompressible, but the presence of Lorentz forces on dust means the transverse magnetic field fluctuations interact with the longitudinal dust velocities, generating a strong compressible dust response.

The gyro-resonant RDI families have a fundamentally different character. These have their fastest growth when the combination of drift velocity and gas wave phase velocity matches the gyro velocity along the same direction, i.e. $\left|\mathbf{w}_{\mathrm{s}} \cdot \mathbf{k} \pm v_{\mathrm{p}}(\hat{\mathbf{k}}) k\right|=\left\langle t_{\mathrm{L}}\right\rangle^{-1}$. Unlike the MHD-wave RDIs, for a mode propagating in a given direction $\hat{\mathbf{k}}$, this resonance occurs at a single specific wavenumber $k_{\mathrm{gyro}}$. The growth rates are sharply peaked around $k_{\text {gyro }}$ with growth rates $\Im(\omega) \sim \mu^{1 / 2} /\left\langle t_{\mathrm{L}}\right\rangle$, which is again sub-linear in the dust-to-gas ratio, but independent of wavenumber (dependent only on the Larmor time). Thus they are more akin to the Brunt-Väisälä modes discussed in Paper I and Squire \& Hopkins (2017a), or resonant cosmic-ray instabilities (Kulsrud \& Pearce 1969). Because the gyro RDIs must overcome resistance by drag, these modes are only unstable when the Larmor time is shorter than the drag time $(\tau \gtrsim 1)$. The gyro-RDI modes resemble perturbed gyro motion and are only weakly compressible in both dust and gas, primarily involving unstable growth of the dust gyro motion and the perturbed transverse field lines.

In the limit where the Lorentz forces become extremely strongly dominant over drag, the drift is tightly aligned along field lines and various 'parallel' instabilities dominate. For example, if the dust is streaming sufficiently super-Alfvénically, instabilities resembling the cosmic-ray streaming instabilities appear, with growth rates $\sim \mu^{1 / 2} w_{\mathrm{s}} k$ increasing rapidly at short wavelengths.

We consider a broad range of astrophysical applications (Section 9). Based on simple order-of-magnitude estimates, we expect these instabilities to be important in a very broad range of astrophysical systems with dust, including cool-star winds, the solar and other stellar coronae, $\mathrm{SNe}$ explosions and remnants (with qualitatively different behaviors for dust in or around the ejecta at each stage of the initial explosion and subsequent blastwave/remnant evolution), $\mathrm{H}$ II regions, star-forming GMCs and galactic nuclei, AGN obscuring torii and narrow-line regions, the diffuse warm $\left(T \sim 10^{4} \mathrm{~K}\right)$ neutral and ionized medium, and the circum-galactic and inter-galactic medium (in 
regions laden with dust). Over the very broad dynamic range spanned by these systems, growth timescales of the interesting instabilities can be as short as $t_{\text {grow }} \lesssim 0.1 \mathrm{~s}$ or as long as $t_{\text {grow }} \sim 10^{9} \mathrm{yr}$. Most importantly, in many cases, the growth timescales are much shorter than other relevant dynamical times of the systems. The non-linear outcomes are likely to be quite different, depending on both the system properties and which of the various different RDIs is the fastest-growing (dominant) mode. For example, both the 'pressure-free' mode and the magnetosonic-wave RDIs directly source large dust-to-gas fluctuations but in a very different manner: in the pressure-free mode the dust is collected in large scale arcs/shells/planes perpendicular to the drift direction, whereas the magnetosonic-wave RDIs concentrate dust into filaments and 'streamers' aligned with propagation (see Paper II). The 'cosmic ray streaming'-type instability, on the other hand, may excite high-frequency Alfvén waves that scatter grains and realign magnetic fields.

Any of these outcomes could have important implications. Grain-grain collisions, coagulation, shattering, grain-ISM chemistry, molecule formation, grain polarization (alignment of spinning dust with magnetic fields), ISM cooling in dusty gas, launching of radiation-pressuredriven winds (via radiation impinging on dust, in particular), retention of dust grains in regions with strong radiation fields, visual and extinction morphologies of dusty systems, local extinction curve variations, magnetic field structure in around bright sources and in the distant CGM/IGM - each of of these could, in principle, be substantially altered by the instabilities here. Related physics may also play a role in resolving several long-standing observational puzzles in these areas (see e.g. Section 9.2). However, to make detailed predictions and understand the observational consequences of the RDI for these systems, we require numerical simulations, which are the only way to realistically explore the non-linear saturation of the instabilities.

Obvious analytic extensions of the work here include considering non-ideal MHD (as discussed in very limited fashion in Squire \& Hopkins 2017a), relevant when ionization fractions are low (but not vanishingly small, where we would simply have the acoustic case in Paper II). As noted in Section 8, Ohmic resistivity is purely diffusive, but Hall MHD is non-diffusive and produces new wave families that generate new RDIs (e.g. the Whistler RDIs), while a proper treatment of ambipolar diffusion (where important) requires a three-fluid theoretical treatment (with ions, neutrals, and grains). Another possible extension is to include kinetic plasma effects: Braginskii viscosity and conduction are dissipative but anisotropic, so introduce additional terms that depend on the mode angles relative to the fields. As seen in a limited fashion above (see Fig. 7), some modes may be damped, but others not, and new instabilities may appear that relate the RDI to other known instabilities of anisotropically conducting systems. A rich phenomenology of RDIs remains largely unexplored.

\section{ACKNOWLEDGEMENTS}

We would like to thank E. S. Phinney and E. Quataert for helpful discussions, as well as our anonymous referee. Support for PFH \& JS was provided by an Alfred P. Sloan Research Fellowship, NASA ATP Grant NNX14AH35G, and NSF Collaborative Research Grant \#1411920 and CAREER grant \#1455342. JS was funded in part by the Gordon and Betty Moore Foundation through Grant GBMF5076 to Lars Bildsten, Eliot Quataert, and E. Sterl Phinney.

\section{REFERENCES}

Agúndez M., Cernicharo J., Guélin M., 2010, ApJ, 724, L133

Akimkin V. V., Kirsanova M. S., Pavlyuchenkov Y. N., Wiebe D. S., 2015, MNRAS, 449, 440

Akimkin V. V., Kirsanova M. S., Pavlyuchenkov Y. N., Wiebe D. S., 2017, MNRAS, 469, 630

Alexashov D. B., Katushkina O. A., Izmodenov V. V., Akaev P. S., 2016, MNRAS, 458, 2553

Anderson L. D. et al., 2010, A\&A, 518, L99

Anderson L. D. et al., 2012, A\&A, 542, A10

Apai D., Linz H., Henning T., Stecklum B., 2005, A\&A, 434, 987

Baes M., Viaene S., 2016, A\&A, 587, A86

Balick B., Alexander J., Hajian A. R., Terzian Y., Perinotto M, Patriarchi P., 1998, AJ, 116, 360

Balick B., Frank A., 2002, ARA\&A, 40, 439

Balsara D. S., 1996, ApJ, 465, 775

Bell A. R., 2004, MNRAS, 353, 550

Bhat N. D. R., Codes J. M., Camilo F., Nice D. J., Lorimer D. R., 2004, ApJ, 605, 759

Bianchi S., Schneider R., 2007, MNRAS, 378, 973

Braginskii S. I., 1965, Rev. Plasma Phys., 1, 205

Caldwell R. R., Hirata C., Kamionkowski M., 2017, ApJ, 839, 91

Chelouche D., Koester B. P., Bowen D. V., 2007, ApJ, 671, L97

Chiaki G., Schneider R., Nozawa T., Omukai K., Limongi M., Yoshida N., Chieffi A., 2014, MNRAS, 439, 3121

Chiang E., Youdin A. N., 2010, Annu. Rev. Earth Planetary Sci., 38, 493

Choi E., Ostriker J. P., Naab T., Johansson P. H., 2012, ApJ, 754, 125

Cioffi D. F., McKee C. F., Bertschinger E., 1988, ApJ, 334, 252

Cox N. L. J. et al., 2012, A\&A, 537, A35

De Looze I, Barlow M. J., Swinyard B. M., Rho J., Gomez H. L., Matsuura M., Wesson R., 2017, MNRAS, 465, 3309

Deguchi S., 1997, in Habing H. J., Lamers H. J. G. L. M., eds, IAU Symp., Vol. 180, Planetary Nebulae, Kluwer Academic Publishers, Dordrecht, p. 151

Deharveng L. et al., 2015, A\&A, 582, A1

Dopcke G., Glover S. C. O., Clark P. C., Klessen R. S., 2013, ApJ, 766, 103

Draine B. T., 2003, ARA\&A, 41, 241

Draine B. T., 2004, in Pfenniger D., Revaz Y., eds, The Cold Universe, Saas-Fee Advanced Course 32, Springer, Berlin, p. 213 
Draine B. T., 2011, ApJ, 732, 100

Draine B. T., Salpeter E. E., 1979a, ApJ, 231, 438

Draine B. T., Salpeter E. E., 1979b, ApJ, 231, 77

Draine B. T., Sutin B., 1987, ApJ, 320, 803

Elitzur M., Shlosman I., 2006, ApJ, 648, L101

Elmegreen B. G., 1979, ApJ, 232, 729

Fong D., Meixner M., Shah R. Y., 2003, ApJ, 582, L39

Garnett D. R., Dinerstein H. L., 2001, ApJ, 558, 145

Goldsmith P. F., Langer W. D., 1978, ApJ, 222, 881

Gontcharov G. A., 2016, Astron. Lett., 42, 445

Grudić M. Y., Hopkins P. F., Faucher-Giguère C.-A., Quataert E., Murray N., Kereš D., 2018, MNRAS, 475, 3511

Guszejnov D., Krumholz M. R., Hopkins P. F., 2016, MNRAS, 458, 673

Hankins M. J., Lau R. M., Morris M. R., Herter T. L., 2017, ApJ, 837, 79

Hartquist T. W., Havnes O., 1994, Astrophys. Space Sci., 218, 23

Haverkorn M., Spangler S. R., 2013, Space Sci. Rev., 178, 483

Hennawi J. F. et al., 2010, ApJ, 719, 1672

Hirashita H., Kuo T.-M., 2011, MNRAS, 416, 1340

Hoang T., 2017, ApJ, 847, 77

Hönig S. F., Kishimoto M., 2010, A\&A, 523, A27

Hopkins P. F., Torrey P., Faucher-Giguere C.-A., Quataert E., Murray N., 2016, MNRAS, 458, 816

Hopkins P. F. et al., 2018, MNRAS, 477, 1578

Hopkins P. F., 2014, ApJ, 797, 59

Hopkins P. F., Conroy C., 2017, ApJ, 835, 154

Hopkins P. F., Squire J., 2017, MNRAS, preprint (arXiv:1707.02997)

Hopkins P. F., Quataert E., Murray N., 2011, MNRAS, 417, 950

Ishibashi W., Fabian A. C., 2016, MNRAS, 457, 2864

Jacquet E., Balbus S., Latter H., 2011, MNRAS, 415, 3591

Ji A. P., Frebel A., Bromm V., 2014, ApJ, 782, 95

Kandel D., Lazarian A., Pogosyan D., 2018, MNRAS, 478, 530

Kim C.-G., Ostriker E. C., 2015, ApJ, 802, 99

Kimura H., Mann I., 1998, Earth Planets Space, 50, 493

Kimura H., Mann I., Mukai T., 1998, Planetary Space Sci., 46, 911

Kobayashi H., Watanabe S.-i., Kimura H., Yamamoto T., 2009, Icarus, 201, 395

Kral Q. et al., 2017, Astron. Rev., 13, 69

Krolik J. H., Begelman M. C., 1988, ApJ, 329, 702

Krüger H., Strub P., Grün E., Sterken V. J., 2015, ApJ, 812, 139

Krumholz M. R., Klein R. I., McKee C. F., 2007, ApJ, 656, 959

Kulsrud R., Pearce W. P., 1969, ApJ, 156, 445

Leccardi A., Molendi S., 2008, A\&A, 487, 461

MacGregor K. B., Stencel R. E., 1992, ApJ, 397, 644

Mann I. et al., 2004, Space Sci. Rev., 110, 269

Mann I., Kohler M., Kimura H., Cechowski A., Minato T., 2006, A\&AR, 13, 159

Mann I., Krivov A., Kimura H., 2000, Icarus, 146, 568

Martin C. L. et al., 2010, ApJ, 721, 174

Martizzi D., Faucher-Giguère C.-A., Quataert E., 2015, MNRAS, 450, 504

Matsuura M. et al., 2009, ApJ, 700, 1067

Matsuura M. et al., 2015, ApJ, 800, 50

McKenzie J. F., Voelk H. J., 1982, A\&A, 116, 191

McKernan B., Yaqoob T., 1998, ApJ, 501, L29+

Ménard B., Fukugita M., 2012, ApJ, 754, 116

Ménard B., Scranton R., Fukugita M., Richards G., 2010, MNRAS, 405, 1025

Miller L., Turner T. J., Reeves J. N., 2008, A\&A, 483, 437

Mor R., Netzer H., Elitzur M., 2009, ApJ, 705, 298

Morris M., 1993, in Schwarz H. E., ed., European Southern Observatory Conference and Workshop Proceedings, Vol. 46, European Southern Observatory Conference and Workshop Proceedings, ESO, Garching, p. 60

Morse J. A., Davidson K., Bally J., Ebbets J., Balick B., Frank A., 1998, AJ, 116, 2443

Mukai T. et al., 1974, PASJ, 26, 445

Mukai T., Yamamoto T., 1979, PASJ, 31, 585

Murray N., Quataert E., Thompson T. A., 2005, ApJ, 618, 569

Nenkova M., Sirocky M. M., Ivezić Ž., Elitzur M., 2008, ApJ, 685, 147

O’Dell C. R., Balick B., Hajian A. R., Henney W. J., Burkert A., 2002, AJ, 123, 3329

O’dell C. R., Handron K. D., 1996, AJ, 111, 1630

Ohgaito R., Mann I., Kuhn J. R., MacQueen R. M. , Kimura H., 2002, ApJ, 578, 610

Owen P. J., Barlow M. J., 2015, ApJ, 801, 141

Padoan P., Cambresy L., Juvela M., Kritsuk A., Langer W. D. , Norman M. L., 2006, ApJ, 649, 807

Peek J. E. G., Ménard B., Corrales L., 2015, ApJ, 813, 7

Prasad C. D., 1995, Solar Phys, 159, 181

Ragot B. R., Kahler S. W., 2003, ApJ, 594, 1049 
Relaño M. et al., 2016, A\&A, 595, A43

Reynolds S. P., Gaensler B. M., Bocchino F., 2012, Space Sci. Revi, 166, 231

Richings A. J., Faucher-Giguère C.-A., 2018, MNRAS, 474, 3673

Riquelme M. A., Spitkovsky A., 2009, ApJ, 694, 626

Risaliti G., Elvis M., Nicastro F., 2002, ApJ, 571, 234

Roth N., Kasen D., Hopkins P. F., Quataert E., 2012, ApJ, 759, 36

Sarangi A., Cherchneff I., 2015, A\&A, 575, A95

Schlafly E. F. et al., 2016, ApJ, 821, 78

Shields J. C., Kennicutt R. C., Jr, 1995, ApJ, 454, 807

Shopov Y. Y. et al., 2008, J. Atmosph. Solar-Terrestr. Phys., 70, 356

Shull J. M., 1978, ApJ, 226, 858

Silvia D. W., Smith B. D., Shull J. M., 2010, ApJ, 715, 1575

Squire J., Hopkins P. F., 2018a, MNRAS, 477, 5011

Squire J., Hopkins P. F., 2018b, ApJ, 856, L15

Strub P., Krüger H., Sterken V. J., 2015, ApJ, 812, 140

Temim T. et al., 2017, ApJ, 836, 129

Thompson T. A., Quataert E., Murray N., 2005, ApJ, 630, 167

Thornton K., Gaudlitz M., Janka H., Steinmetz M., 1998, ApJ, 500, 95

Tielens A. G. G. M., 2005, The Physics and Chemistry of the Interstellar Medium. Cambridge University Press, Cambridge, UK

Topchieva A., Wiebe D., Kirsanova M., Krushinsky V., 2017, in Balega Y. Y., Kudryavtsev D. O., Romanyuk I. I., Yakunin I. A., eds, ASP Conf. Ser., Vol.

510, Stars: From Collapse to Collapse, Astronomical Society of the Pacific, San Francisco, p. 98

Tumlinson J., Peeples M. S., Werk J. K., 2017, ARA\&A, 55, 389

Wada K., Papadopoulos P., Spaans M., 2009, ApJ, 702, 63

Walch S., Naab T., 2015, MNRAS, 451, 2757

Weingartner J. C., Draine B. T., 2001a, ApJ, 563, 842

Weingartner J. C., Draine B. T., 2001b, ApJ, 553, 581

Weingartner J. C., Draine B. T., 2001c, ApJS, 134, 263

Wentzel D. G., 1969, ApJ, 156, 303

Winters J. M., Dominik C., Sedlmayr E., 1994, A\&A, 288, 255

Woitke P., 2006, A\&A, 460, L9

Youdin A. N., Goodman J., 2005, ApJ, 620, 459

Young P. A., Highberger J. L., Arnett D., Ziurys L. M., 2003, ApJ, 597, L53

Ziurys L. M., Milam S. N., Apponi A. J., Woolf N. J., 2007, Nature, 447, 1094

\section{APPENDIX A: DISPERSION RELATION}

To obtain the dispersion relation, begin with the linearized equations (equation 4$),{ }^{31}$ and then make the Fourier ansatz as in the text, $\left.\delta X=\delta X_{0} \exp [i(\mathbf{k} \cdot \mathbf{x}-\omega t)]\right)$. Use the divergence constraint $0=\nabla \cdot \delta \mathbf{B}=i \mathbf{k} \cdot \delta \mathbf{B}$ to eliminate one component of $\delta \mathbf{B}\left(\delta \mathbf{B}_{z}=-\left(k_{x} \delta \mathbf{B}_{x}+\right.\right.$ $\left.k_{y} \delta \mathbf{B}_{y}\right) / k_{z}$ ), where we define the axes $\hat{x}, \hat{y}, \hat{z}$ as the unit vectors parallel to $\left(\mathbf{w}_{\mathrm{s}} \times \mathbf{B}_{0}\right) \times \mathbf{w}_{\mathrm{s}}, \mathbf{w}_{\mathrm{s}} \times \mathbf{B}_{0}$, and $\mathbf{w}_{\mathrm{s}}$, respectively. For convenience we will make the problem dimensionless, by defining the units of density, velocity, and time equal to the homogeneous values of $\rho_{0}, c_{\mathrm{s}}$, and $\left\langle t_{\mathrm{s}}\right\rangle$; we also conveniently write $\delta \mathbf{B}$ in units of $\left|\mathbf{B}_{0}\right|$.

Inserting a single mode, equation (4) can be written: $\omega \mathbf{X}=\mathbb{T} \mathbf{X}$, where $\mathbf{X}=\left(\delta \rho_{\mathrm{d}}, \delta \mathbf{v}_{x}, \delta \mathbf{v}_{y}, \delta \mathbf{v}_{z}, \delta \rho, \delta \mathbf{u}_{x}, \delta \mathbf{u}_{y}, \delta \mathbf{u}_{z}, \delta \mathbf{B}_{x}, \delta \mathbf{B}_{y}\right)$ (recall we eliminated $\delta \mathbf{B}_{z}$ already), and the matrix $\mathbb{T}$ is given by

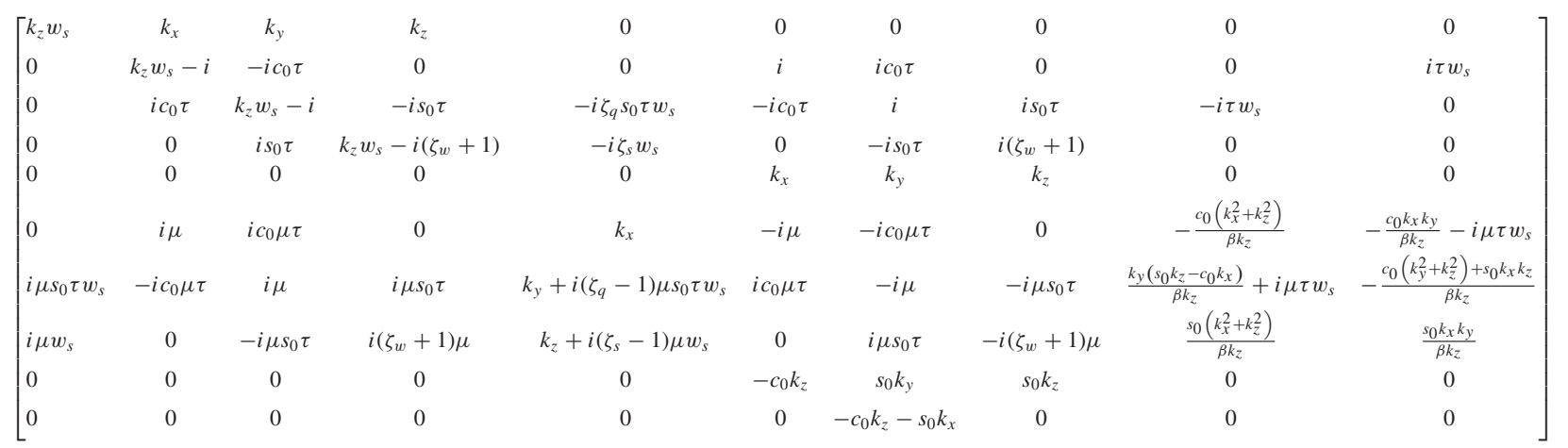

where $c_{0} \equiv \cos \theta_{\mathbf{B w}}=\hat{\mathbf{B}}_{0} \cdot \hat{\mathbf{w}}_{\mathrm{s}}$ and $s_{0} \equiv\left(1-c_{0}^{2}\right)^{1 / 2}$.

The solutions $\omega$ are the eigenvalues of $\mathbb{T}$. We can write the full dispersion relation as the characteristic polynomial of this matrix, but the resulting 10th-order polynomial is not instructive. We instead focus in the text on numerical solutions to the general equation, and intuitive analytic expressions that apply under appropriate limits.

\footnotetext{
${ }^{31}$ For stratified media, we can explicitly include a pressure equation $D \delta P / D t=c_{\mathrm{s}}^{2} D \delta \rho / D t$ (where $D / D t$ is the comoving derivative) but for our homogeneous background this trivially evaluates to $\delta P=c_{\mathrm{s}}^{2} \delta \rho$ so we simply insert this directly in equation (4).
} 
Since this is a 10x10 sparse matrix and we consider limits where some parameters are much larger than others, in numerically evaluating $\omega$, care is needed. Numerical solutions in the text use the Python MPMATH package which allows for arbitrary floating-point precision, retaining 30 significant figures, and were subsequently verified directly.

\section{APPENDIX B: DUST AND GAS RESONANCES}

In this appendix, we discuss the origin of the mid- $k$, high- $k$, and gyroresonant modes, and how these relate to the matrix theory introduced in Paper I. Our starting point is the full dust-gas matrix operator, equation (A1), and we use the dimensionless units of Section A. Because we have organized the variables as $\mathbf{X}=\left(\mathbf{X}_{\mathrm{d}}, \mathbf{X}_{\mathrm{g}}\right)$, where $\mathbf{X}_{\mathrm{d}}$ and $\mathbf{X}_{\mathrm{g}}$ represent the dust and gas variables, respectively, $\mathbb{T}$ has the form,

$\mathbb{T}=\mathbb{T}_{0}+\mu \mathbb{T}^{(1)}=\left(\begin{array}{cc}\mathcal{A} & \mathcal{C} \\ 0 & \mathcal{F}\end{array}\right)+\mu\left(\begin{array}{cc}\mathcal{T}_{A A}^{(1)} & \mathcal{T}_{A F}^{(1)} \\ \mathcal{T}_{F A}^{(1)} & \mathcal{T}_{F F}^{(1)}\end{array}\right)$

where $\mathcal{A}$ (the top-left $4 \times 4$ block in equation A1) represents the effect of the dust on the dust itself, $\mathcal{F}$ the effect of the gas on the gas, $\mathcal{C}$ the effect of the gas on the dust, and $\mathcal{T}_{F A}^{(1)}$ the backreaction from the dust back onto the gas. We will calculate the eigenvalues of equation (B1) by considering $\mu \mathbb{T}^{(1)}$ (with $\mu \ll 1$ ) to be a perturbation to $\mathbb{T}_{0}$, analyzing the resulting perturbation $\left(\omega^{(1)}\right.$ ) to the eigenvalues of $\mathbb{T}_{0}\left(\right.$ termed $\omega_{0}$ ) using perturbation theory. In other words, we shall find $\omega$, the eigenvalue of $\mathbb{T}$, through the expansion

$\omega=\omega_{0}+\omega^{(1)}+\cdots$,

where $\omega^{(1)}$ scales with some power of $\mu$. The basic result of Paper I was that if $\mathcal{A}$ and $\mathcal{F}$ share an eigenmode (i.e. there is a resonance), then the system is likely unstable, $\Im\left(\omega^{(1)}\right) \neq 0$, and that $\omega^{(1)}$ (which determines the growth rate of the resulting instability) scales as $\omega^{(1)} \sim \mu^{1 / 2}$ (or $\left.\omega^{(1)} \sim \mu^{1 / 3}\right)$, rather than the usual perturbation theory expectation, $\omega^{(1)} \sim \mu$. In this work, we have extended the dust model used by Paper I to account for charge on the grains. This changes matrix $\mathcal{A}$, allowing a richer mode structure and the appearance of the gyro-resonance modes in the dust. We thus straightforwardly extend the theory of Paper I to account for this more complex dust physics.

Before continuing, we caution that these ideas only apply at sufficiently small $\mu$ such that $\mu \mathcal{T}^{(1)}$ can be considered a perturbation. With so many combinations of parameters, even values one might usually consider 'small' (e.g. $\mu \sim 0.01$ ) may not be sufficiently small, and non-resonant modes can dominate in certain regimes (for instance, the low- $k$, 'pressure-free' modes discussed in Section 4.1 fall into this category). This is particularly true at high $\tau$, where the full-mode structure (at arbitrary $\mu, k, w_{\mathrm{s}} / c_{\mathrm{s}}, v_{\mathrm{A}} / c_{\mathrm{s}}$ etc.) becomes very complex and difficult to classify; see e.g. Sections $4.2-4.3$ for some examples.

\section{B1 Dust eigenmode structure}

A key aspect of understanding the structure of the RDI solutions to the full matrix equation (A1) is understanding the eigenmodes of the dust matrix $\mathcal{A}$. This can be written in the form

$\mathcal{A}=\left(\begin{array}{cc}k_{z} w_{\mathrm{s}} & \mathbf{k}^{T} \\ \mathbf{0} & k_{z} w_{\mathrm{s}} \rrbracket+\mathcal{D}_{\text {drag }}+\tau \mathcal{D}_{\text {gyro }}\end{array}\right)$.

The submatrices $\mathcal{D}_{\text {drag }}$ and $\tau \mathcal{D}_{\text {gyro }}$ describe, respectively, the effect of drag forces on the dust (i.e. the $-(\delta \mathbf{v}-\delta \mathbf{u}) /\left\langle t_{\mathrm{s}}\right\rangle$ term in equation $\left.(4)\right)$

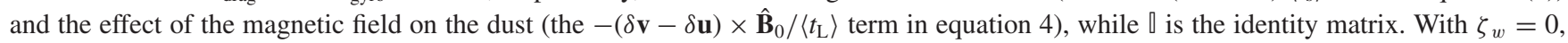
large- $\tau$, or for various special angles (e.g. $\cos ^{2} \theta_{\mathbf{B w}}=1$ ), the eigenvalues of $\mathcal{A}$ are particularly simple ${ }^{32}$; see Section 6 . For example, with $\zeta_{w}=0$ (i.e. when $t_{\mathrm{s}}$ is independent of $|\mathbf{v}-\mathbf{u}|$ ) they are: (i) a dust density perturbation, with no associated velocity perturbation and frequency $\omega_{\mathcal{A}}=k_{z} w_{\mathrm{s}}$ (due to the Doppler shift from $\mathbf{w}_{\mathrm{s}}$ ); (ii) a damped velocity perturbation along the magnetic field with $\omega_{\mathcal{A}}=k_{z} w_{\mathrm{s}}-i$ (recall that, because we work in units where $\left\langle t_{\mathrm{s}}\right\rangle=1$, this is damping at the rate $\Im(\omega)=-\left\langle t_{\mathrm{s}}\right\rangle^{-1}$ ); and (iii) two damped gyration modes, which involve magnetic-field-influenced motion perpendicular to the field and have $\omega_{\mathcal{A}}=k_{z} w_{\mathrm{s}} \pm \tau-i$.

\section{B2 Resonance between the dust and the fluid}

The matrix-resonance theory of Paper I then says that we should attempt to match eigenmodes of the gas, denoted here by $\omega_{\mathcal{F}}$, to those of $\mathcal{A}$, so as to find the regions of parameter space where $\omega^{(1)}$ is largest (i.e. where any instability grows the fastest). As discussed in Section 5.1, the fluid part of equation (A1) (the bottom-right $6 \times 6$ block) supports six real oscillation modes (eigenmodes): shear-Alfvén waves with $\omega_{\mathcal{F}}= \pm \mathbf{v}_{A} \cdot \mathbf{k}$, slow waves with $\omega_{\mathcal{F}}= \pm \mathbf{v}_{-} \cdot \mathbf{k}$, and fast waves with $\omega_{\mathcal{F}}= \pm \mathbf{v}_{+} \cdot \mathbf{k}$. To make progress, we must choose one of these eigenmodes and match it to a dust eigenmode $\omega_{\mathcal{A}} \approx \omega_{\mathcal{F}}$. For the remainder of this section, we shall assume that we have done this, and denote the mode's eigenvalue by $\omega_{\mathcal{F}}$ and the corresponding left and right eigenmodes by $\xi_{\mathcal{F}}^{L}$ and $\xi_{\mathcal{F}}^{R}$ respectively (these satisfy $\xi_{\mathcal{F}}^{L}\left(\mathcal{F}-\omega_{\mathcal{F}} \llbracket\right.$ ) and $\left(\mathcal{F}-\omega_{\mathcal{F}} \rrbracket\right) \xi_{\mathcal{F}}^{R}$, as well as $\left.\xi_{\mathcal{F}}^{L} \mathcal{F} \xi_{\mathcal{F}}^{R}=\omega_{\mathcal{F}}\right)$.

\footnotetext{
${ }^{32}$ In the general case (e.g. arbitrary $\tau$ or $\zeta_{w}$ ) the fundamental character of the eigenvalue solutions is unchanged, but their form becomes complicated and unintuitive.
} 
Let us consider the dust modes $\left(\omega_{\mathcal{A}}\right)$, so as to understand the different regimes of the MHD RDI. The dust density mode, which satisfies $\omega_{\mathcal{A}}=k_{z} w_{\mathrm{s}}$, is the simplest: the resonance condition $\omega_{\mathcal{F}}=\omega_{\mathcal{A}}=\mathbf{k} \cdot \mathbf{w}_{\mathrm{s}}$ can generally be satisfied exactly for some choice of $\hat{\mathbf{k}}$, leading to the standard 'mid- $k$ ' RDI, described in Section 5.3. The other dust modes, which involve a velocity perturbation, are damped by the drag on the gas (with $\Im\left(\omega_{\mathcal{A}}\right) \sim-\left\langle t_{\mathrm{s}}\right\rangle^{-1}$ ), and thus cannot resonate exactly with the (undamped) MHD modes. However, it transpires that we may consider $\mathcal{D}_{\text {drag }}$ to be a part of the perturbation (i.e. effectively part of $\mu \mathbb{T}^{(1)}$ ), so long as the perturbation to the eigenmode ( $\omega^{(1)}$ ) is larger than the change to $\omega_{\mathcal{A}}$ that arises from including $\mathcal{D}_{\text {drag. }}$ In other words, if part of the $\mathcal{A}$ matrix $\left(\mathcal{D}_{\text {drag }}\right.$ or $\left.\mathcal{D}_{\text {gyro }}\right)$ is smaller than $\mu \mathbb{T}^{(1)}$, we should consider this part as belonging to the perturbation, rather than to the $\mathcal{A}$ matrix itself. ${ }^{33}$ This implies that if $\omega^{(1)} \gtrsim 1-$ i.e. if the perturbation to the eigenvalues from $\mathbb{T}^{(1)}$ is larger than that due to $\mathcal{D}_{\text {drag }}$ - then we should not include $\mathcal{D}_{\text {drag }}$ in the calculation of the eigenmodes of $\mathcal{A}$ (physically, this condition, $\omega^{(1)} \gtrsim 1=\left\langle t_{\mathrm{s}}\right\rangle^{-1}$, is simply that the instability growth time is faster than the stopping time). We then see that the gyroresonant modes occur when $\pm \tau+\mathbf{k} \cdot \mathbf{w}_{\mathrm{s}} \approx \omega_{F}$ (see equation 43 ), and when the resulting perturbation, $\omega^{(1)}$, satisfies $\omega^{(1)} \gtrsim 1$ (see Section 6 for further discussion). This is only possible for $\tau>1$, because otherwise the damping of the dust gyration modes is larger than the effect of the magnetic field (i.e. the gyration). The high- $k$ RDI (Section 5.4) is slightly different, arising from a triple resonance between the fluid mode, the dust density perturbation mode $\left(\omega_{\mathcal{A}}=\mathbf{k} \cdot \mathbf{w}_{\mathrm{s}}\right)$, and the (damped) dust velocity perturbation mode $\left(\omega_{\mathcal{A}}=\mathbf{k} \cdot \mathbf{w}_{\mathrm{s}}-i\right)$. The matrix is then triply defective, leading to the different high- $k$ RDI scaling, $\omega^{(1)} \sim \mu^{1 / 3}\left(\right.$ rather than $\omega^{(1)} \sim \mu^{1 / 2} ;$ see Paper I). It transpires ${ }^{33}$ that this triple resonance requires that the $\omega^{(1)}$ perturbation is larger than both $\mathcal{D}_{\text {drag }}$ and $\mathcal{D}_{\text {gyro }}$, so the high- $k$ scaling applies once $\omega^{(1)} \gtrsim \operatorname{MAX}(1, \tau)$. When $\tau>1$ there is a transition range $1 \lesssim \omega^{(1)} \lesssim \tau$ where there is no clear universal scaling of the mode; see, for example, the $\tau=100$ cases in Fig. 2 (in particular the fast-mode panel, which reaches sufficiently high growth rates to show the $\omega^{(1)} \gtrsim \tau$ transition).

Once one has decided which of the modes one wishes to study, the growth rate can be computed using the techniques introduced in Paper I. For the mid- $k$ RDI and the gyro-resonance mode, which arise from the double resonance (between one dust eigenmode and the chosen gas eigenmode), the eigenvalue perturbation is given by

$\omega^{(1)} \approx \mu^{1 / 2}\left[\left(\xi_{\mathcal{F}}^{\mathrm{L}} \mathcal{T}_{F A}^{(1)} \xi_{\mathcal{A}}^{\mathrm{R}}\right)\left(\xi_{\mathcal{A}}^{\mathrm{L}} \mathcal{C} \xi_{\mathcal{F}}^{\mathrm{R}}\right)\right]^{1 / 2}$,

where $\xi_{\mathcal{A}}^{\mathrm{L}}$ and $\xi_{\mathcal{A}}^{\mathrm{R}}$ are the left and right eigenvectors for the chosen dust mode (mid- $k$ RDI or gyro-resonance mode). For the high- $k$ RDI, which arises from the triple resonance (between two dust eigenmodes and the chosen gas eigenmodes), the perturbation is

$\omega^{(1)} \approx s_{3} \mu^{1 / 3}\left[\left(\xi \xi_{\mathcal{F}}^{\mathrm{L}} \mathcal{T}_{\rho_{d}}^{(1)}\right)\left(\mathbf{k}^{T} \mathcal{C}_{\mathbf{v}} \xi_{\mathcal{F}}^{\mathrm{R}}\right)\right]^{1 / 3}$

where $\mathcal{T}_{\rho_{d}}^{(1)}$ is the left column of $\mathcal{T}_{F A}^{(1)}$ (this arises from the $\omega_{\mathcal{A}}=k_{z} w_{\mathrm{s}}$ dust eigenmode), $\mathcal{C}_{\mathbf{v}}$ denotes the lower three columns of $\mathcal{C}$, and we have also assumed $\omega^{(1)} \gg \tau$ for simplicity (so as to easily compute the dust eigenmodes). Such methods - i.e. using equations (B5) and (B6) - are commensurate with the dispersion-relation expansions used throughout the main text: in some cases these methods provide a simpler way of obtaining the RDI growth rates, in other cases the dispersion-relation expansions are simpler. While equation (B5) (for both the mid- $k$ and gyro-resonant mode) and equation (B6) can be straightforwardly, if tediously, computed from the matrix equation (A1), the resulting expressions are complex enough so as to require subsidiary expansions to reach forms similar to those given in the main text. Given this, we do not provide these expressions in full here.

\section{B3 An algorithm to find magnetic RDIs}

Let us summarize the rather technical discussion of the previous paragraphs with a simple algorithm for 'choosing' the relevant dust-gas resonance, using the matrix resonance theory of Paper I.

(i) Choose a gas wave and calculate its eigenfrequencies and eigenmodes.

(ii) Calculate the perturbed eigenvalues $\omega^{(1)}$ of the mid- $k$, high- $k$, and gyroresonant modes from equations, and gyroresonant modes from Eqs. equationsB5) and (B6). (Note that the low- $k$ modes do not arise from a resonance at all; see Section 4.1 and Paper II).

(iii) If the gyroresonant mode satisfies $\omega^{(1)} \gtrsim 1$ then it can grow, and the expression is valid. If not, it is damped by the gas drag (i.e. the neglected $\mathcal{D}_{\text {drag }}$ term in $\mathcal{A}$ is important).

(iv) If the mid- $k$ RDI (equation B5) satisfies $\omega^{(1)} \lesssim 1$, then this is the correct expression and the mode is in the mid- $k$ regime. Otherwise, if the high- $k$ result (equation B6) satisfies $\omega^{(1)} \gtrsim \tau$, the expression is valid and the mode is in the high- $k$ regime. If $1 \lesssim \omega^{(1)} \lesssim \tau$, it is likely that neither the mid- $k$ or the high- $k$ results are correct.

${ }^{33}$ The easiest way to understand that this should be the case is to put the matrix (B1) into the fluid eigenmode basis, by making the transformation,

$\mathbb{T}_{\mathcal{F}}=\left(\begin{array}{cc}\square & 0 \\ 0 & \xi_{\mathcal{F}}^{L}\end{array}\right) \mathbb{U}\left(\begin{array}{cc}\square & 0 \\ 0 & \xi_{\mathcal{F}}^{R}\end{array}\right)=\left(\begin{array}{ll}\mathcal{A} & \mathcal{C} \xi_{\mathcal{F}}^{R} \\ \mu \xi_{\mathcal{F}}^{L} \mathcal{T}_{F A}^{(1)} & \omega_{\mathcal{F}}\end{array}\right)$.

Note that we have included only the $\mathcal{T}_{F A}^{(1)}$ part of $\mathbb{T}^{(1)}$ in equation (B4), as appropriate for computation of the lowest-order $\mu$ perturbation (see Paper I). A direct computation of the determinant of $\mathbb{T}_{\mathcal{F}}-\left(\omega_{\mathcal{F}}+\omega^{(1)}\right) \llbracket$ captures both the mid- $k$ and high- $k$ RDI growth rates (and the gyroresonant mode). Specifically, if $\omega^{(1)}$ $\ll 1$, the $\mathcal{D}_{\text {drag }}$ contribution is important and we obtain only the mid- $k$ mode. If $\omega^{(1)} \gg 1, \mathcal{D}_{\text {drag }}$ does not contribute and we can obtain the gyro-resonant mode (as a different root of the resulting polynomial in $\omega^{(1)}$ ). Finally, if $\omega^{(1)} \gg \operatorname{MAX}(1, \tau)$, neither $\mathcal{D}_{\text {drag }}$ nor $\mathcal{D}_{\text {gyro }}$ contributes, and we obtain the high- $k$ mode $\omega^{(1)} \sim \mu^{1 / 3}$. 
This series of steps can usually be used to understand the transitions between regimes discussed in Sections 5.3-5.4 and Section 6, although inaccuracies can arise near certain special points in parameter space (e.g. for certain combinations of the $\zeta_{X}$ parameters). Most importantly, it shows that the transition between the mid- $k\left(\Im(\omega) \sim k^{1 / 2}\right)$ and high- $k\left(\Im(\omega) \sim k^{1 / 3}\right)$ regimes occurs when $\Im(\omega) \sim\left\langle t_{\mathrm{s}}\right\rangle^{-1}$ if $\tau \lesssim$ 1 , and that the gyro-resonant mode always requires $\tau \gtrsim 1$ to grow. The method does not, however, find non-resonant modes (e.g. the Bell instability in Section 4.3, or the low- $k$ modes of Section 4.1), which may be the fastest-growing modes in some regimes (e.g. $\tau \gg 1$ and/or larger $\mu$ ).

This paper has been typeset from a $\mathrm{T}_{\mathrm{E}} \mathrm{X} / \mathrm{LAT} \mathrm{E} \mathrm{X}$ file prepared by the author. 